\title{
CASES OF RESILIENCY: EXPLORING EXPERIENCES OF REFUGEE WOMEN PARTICIPATING IN U.S. TEXTILE AND APPAREL NONPROFIT ORGANIZATIONS' SKILLS TRAINING PROGRAMS.
}

A Dissertation
presented to
the Faculty of the Graduate School
at the University of Missouri-Columbia
In Partial Fulfillment
of the Requirements for the Degree
Doctor of Philosophy
by ANGELA URIYO
Dr. Pamela Norum, Dissertation Supervisor
JULY 2018


(C) Copyright by Angela Uriyo 2018

All Rights Reserved 
The undersigned, appointed by the dean of the Graduate School, have examined the dissertation entitled

\section{CASES OF RESILIENCY: EXPLORING EXPERIENCES OF REFUGEE WOMEN PARTICIPATING IN U.S. TEXTILE AND APPAREL NONPROFIT ORGANIZATIONS' SKILLS TRAINING PROGRAMS.}

presented by Angela Uriyo, a candidate for the degree of doctor of philosophy, and hereby certify that, in their opinion, it is worthy of acceptance.

Dr. Pamela Norum

Dr. Jung Ha-Brookshire

Dr. Kristen Morris

Dr. Yong Volz 
For my family, friends, and community who encouraged, prayed, advised, and financially supported me-making my dream come to fruition.

Yann, Yohann, Charlie and Josiah-thank you for accompanying me along every twist and turn on this journey.

Baba and Mama - may you enjoy the fruits of the seeds you planted in my life and carefully supported oh so many years ago. 


\section{ACKNOWLEDGEMENTS}

I begin by first acknowledging the Lord God my creator who makes all things possible. My faith in His plan for my life is what carried me through this doctoral journey. He is who made me understand that this process is a personal journey.

I am grateful to my advisor, Dr. Norum, for the roles she played as my mentor, guide, and counsel during this four-year process. I would also like to thank Dr. HaBrookshire, who asked the tough questions and challenged me to greater heights in my thought processes. I thank Dr. Volz, who as an expert methodologist, first got me fascinated with the qualitative paradigm of inquiry and was always eager to help and guide me through proper methodological practices. I thank Dr. Morris for always keeping the door to her office open and being willing to freely share her expertise, experience, and resources with me as a way to build-up my knowledge base. I appreciate all their contributions to my scholarly development.

To my friend, Rayna, without whom my research process may have been extended or never realized. I am thankful for your optimism, hard work, and heart to help others within and outside of your community live a better life. You truly helped me believe in the value, importance, and relevance of this research study. Last and not least, thank you to all the participants in this study, who placed their trust in me and shared their lived experiences honestly and openly. Your help proved invaluable to fulfilling the purpose of this research study. 


\section{TABLE OF CONTENTS}

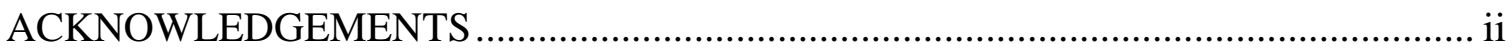

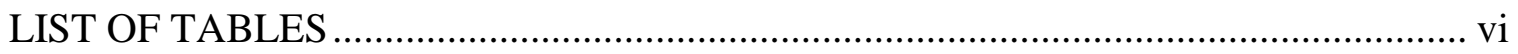

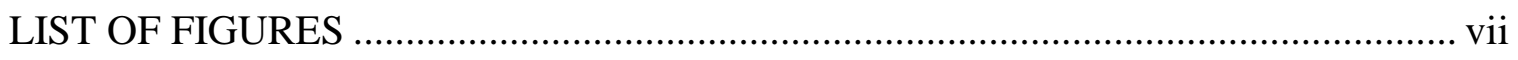

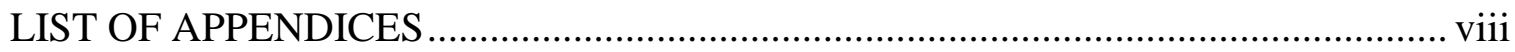

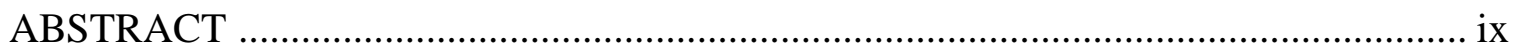

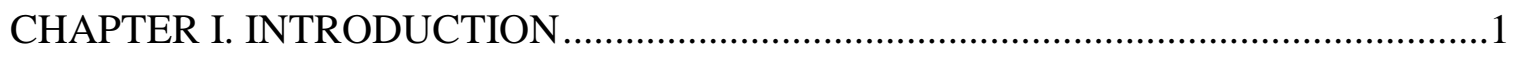

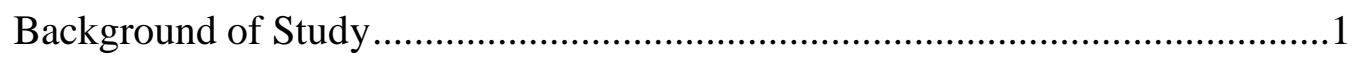

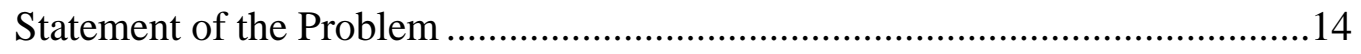

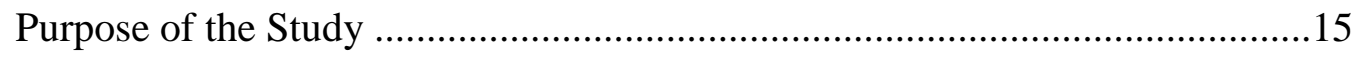

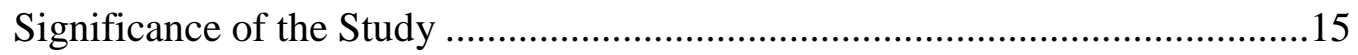

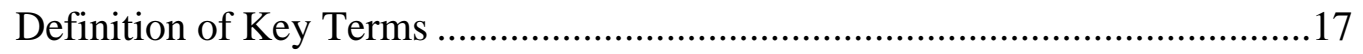

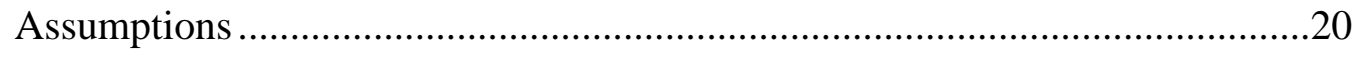

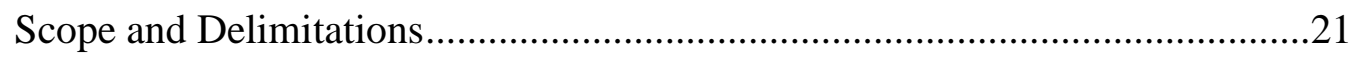

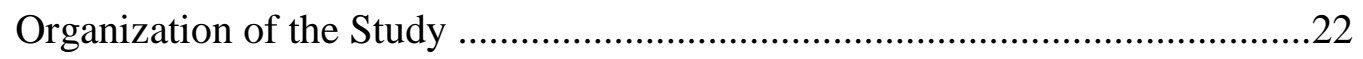

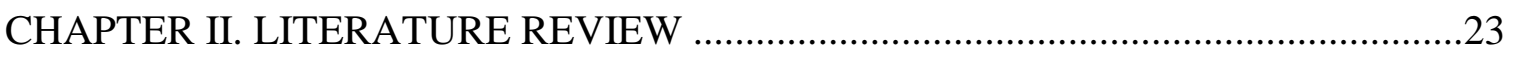

Career Transition \& Training ...............................................................23

Individual Characteristics that Impact the effectiveness of training .................24

Motivations to participate in training ........................................................26

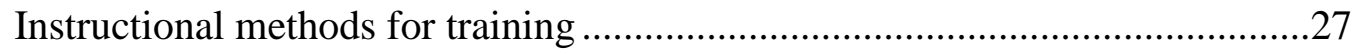

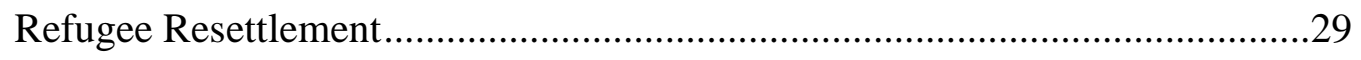

Factors affecting training participation and outcomes ...................................33

Characteristics of female refugees in the United States .................................34

Strategies used to increase refugee employability .....................................40

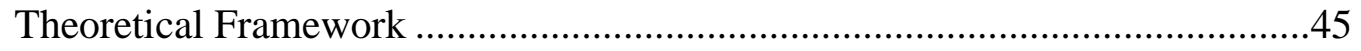


Motivation

Motivation for training .......................................................................4

Theories of Motivations ............................................................................48

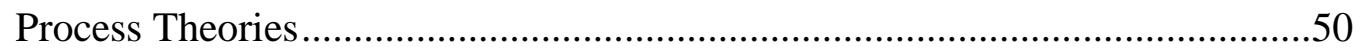

Previous applications of expectancy theory …...........................................53

Research Gap, Purpose of the Study and Research Questions..........................55

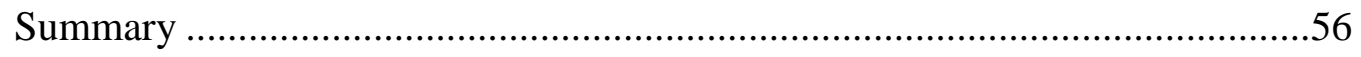

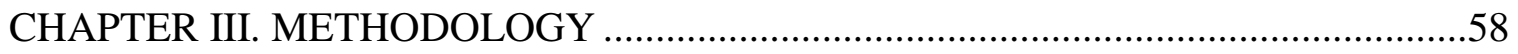

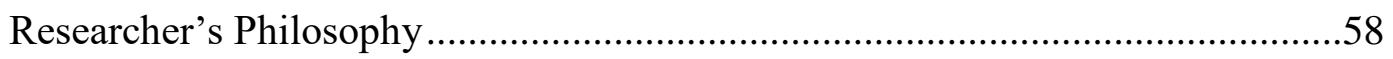

Research Methodology and Design Appropriateness .....................................60

Elaboration and rational for research design ...........................................64

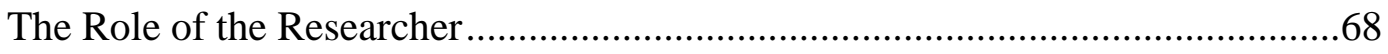

Research Questions ................................................................................... 72

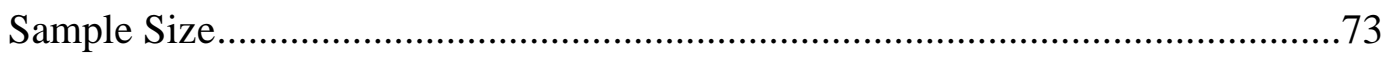

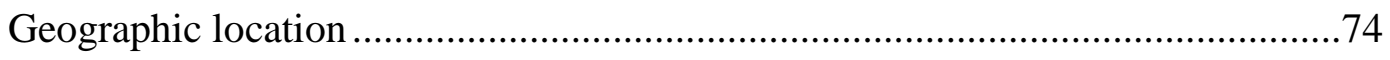

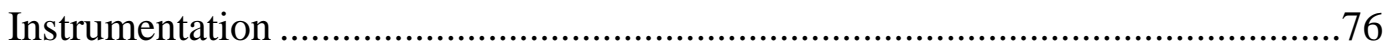

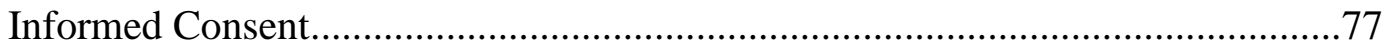

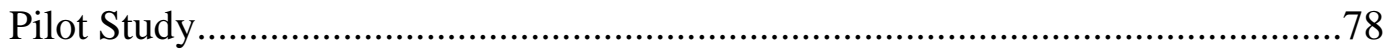

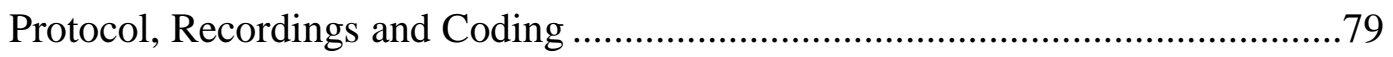

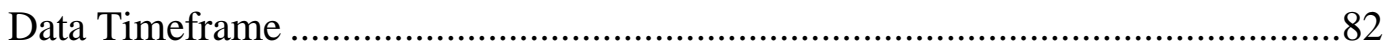

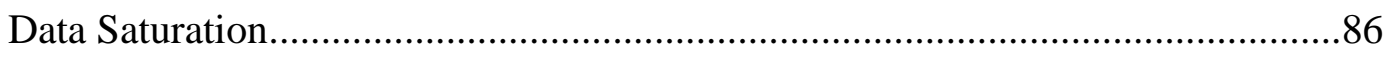

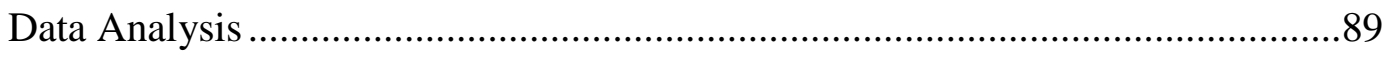

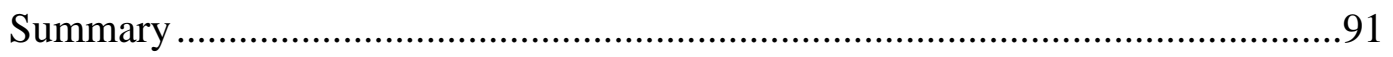

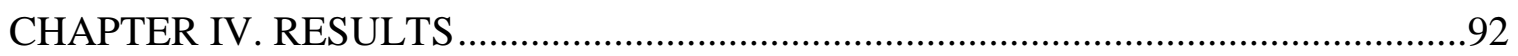

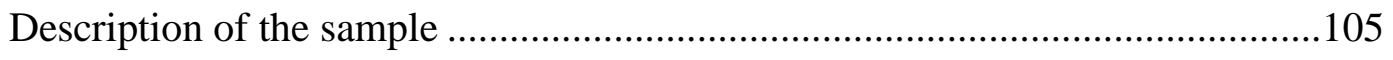


Thematic categories and interpretations 110

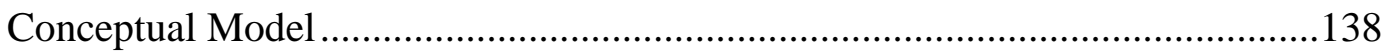

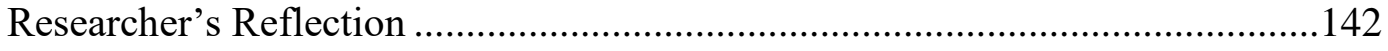

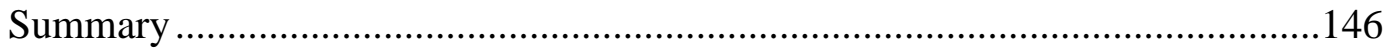

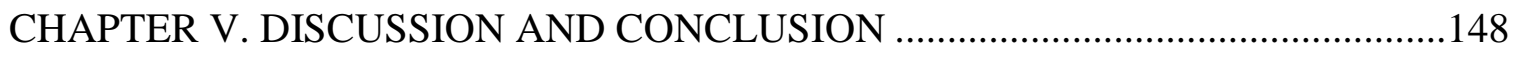

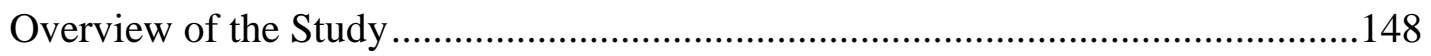

Contributions and Implications for Theory …............................................... 153

Contributions and Implications for Practice ......................................................155

Limitations and Recommendations for Future Study .....................................159

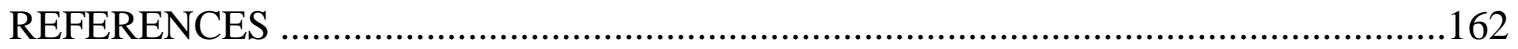

APPENDIX

A. EMAIL RECRUITMENT LETTER .................................................................. 187

B. CONSENT TO ACT AS HUMAN PARTICIPANT -REFUGEES ....................187

C. CONSENT TO ACT AS A HUMAN SUBJECT -LEADERS ............................191

D. INTERVIEW PROTOCOL FOR REFUGEE WOMEN ...................................194

E. INTERVIEW PROTOCOL FOR ORGANIZATIONAL LEADERS .................199

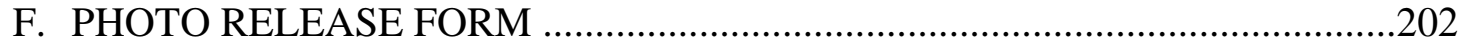

G. OBSERVATIONAL PROTOCOL .................................................................204

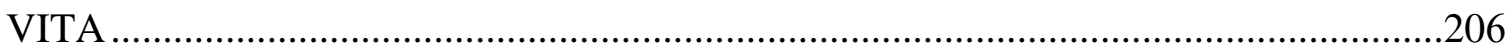




\section{LIST OF TABLES}

Table Page

1. Research questions and corresponding interview question examples...................73

2. Characteristics of textile and apparel NPOs' career training programs ..................93

3. Semi-structured interviews at Avenio's Training Program.................................98

4. Semi-structured interviews at Univo's Training Program ................................101

5. Semi-structured interviews at Leventis’ Training Program ...............................102

6. Semi-structured interviews at Voltan's Training Program .................................104

7. Semi-structured interviews at Salas' Training Program ...................................105

8. Demographic characteristics of trainees with refugee backgrounds ....................106

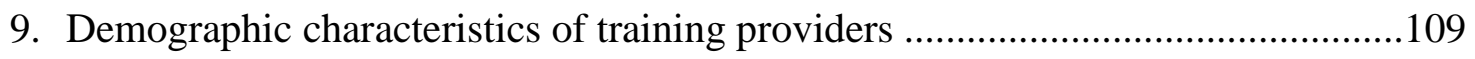




\section{LIST OF FIGURES}

Figure

Page

1. Embedded multiple case design

2. The application of embedded multiple case design ............................................64

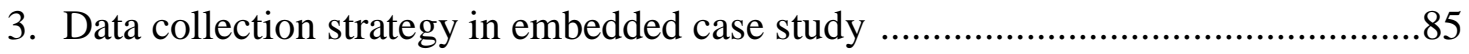

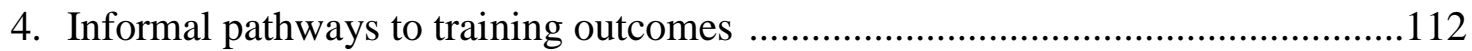

5. A visual representation of how conditional factors in trainees' lives facilitated

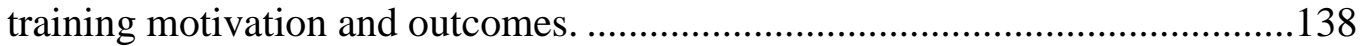




\section{LIST OF APPENDICES}

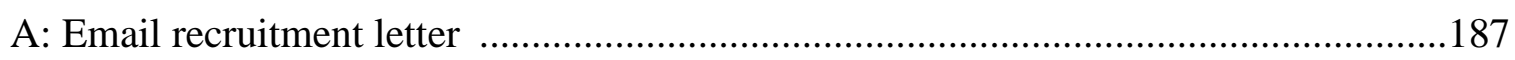

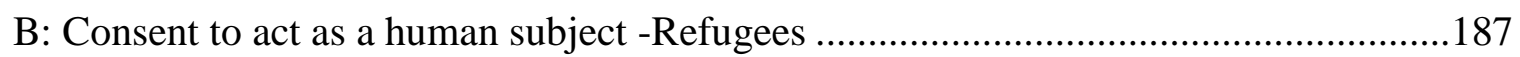

$\mathrm{C}$ : Consent to act as a human subject -Organizational leaders ....................................191

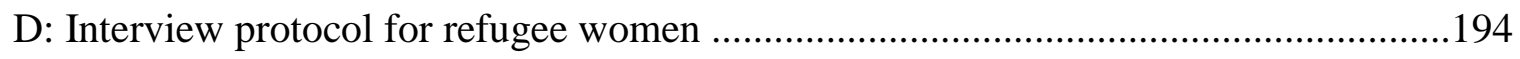

E: Interview protocol for organizational leaders ...............................................199

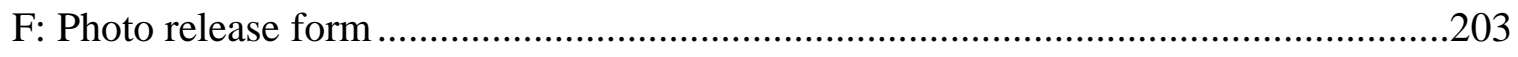

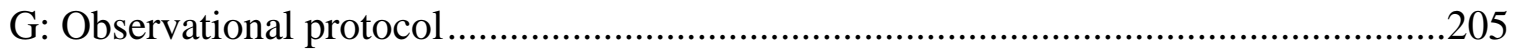




\title{
CASES OF RESILIENCY: EXPLORING EXPERIENCES OF REFUGEE WOMEN PARTICIPATING IN U.S. TEXTILE AND APPAREL NONPROFIT ORGANIZATIONS' SKILLS TRAINING PROGRAMS.
}

\author{
Angela Uriyo
}

Dr. Pamela Norum, Dissertation Supervisor

\begin{abstract}
Humanitarian crises around the world have forced thousands of refugees away from their native lands to neighboring countries on their own continent and as far as Europe and the United States, in search of safe harbor (UNHCR, 2017) and a better life (Igielnik \& Krogstad, 2017). Refugees resettled in the United States, need to become selfsufficient within 18 months of arrival (U.S. Department of State, 2017). With the re-birth of manufacturing in the northwest region of the United States, local textile and apparel nonprofit organizations set-up community-based sewing programs that provided skillsbuilding and job training opportunities to refugees resettled in the region that match the needs of the local labor market, which is essential to their economic integration (Workforce Training and Education Coordinating Board, 2008).

Several empirical studies have pointed out how motivation to participate in trainings impacts the effectiveness of training (Tannenbaum \& Yukl, 1992; Colquitt, LePine \& Noe, 2000). Literature in the career training, refugee resettlement, and clothing and textiles disciplines has not addressed what motivates women of refugee background to participate in career transitions and/or attain training outcomes. Further, it has not defined what the role of U.S. textile and apparel NPOs in the vocational rehabilitation of refugees resettled in the United States is.
\end{abstract}


Therefore, the aim of this study was to gain in-depth understanding of the career transition experiences of women with refugee backgrounds within the context of textile and apparel NPOs in the United States by exploring what motivated them to participate in the training programs and attain training outcomes. Using a multiple case study approach, participant observations, direct observations and in-depth interviews were conducted at five textile and apparel NPO training sites. Fourteen graduate and current trainees as well as 10 training providers in each textile and apparel NPO who were knowledgeable about the organization's structure, vision and mission were interviewed. The data analysis uncovered nine emergent themes that represented factors that facilitated or hindered the motivation of trainees to participate in career transition programs and attain training outcomes. 


\section{CHAPTER I: INTRODUCTION}

This chapter contains the following sections: (a) background of the study, (b) statement of the problem, (c) purpose of the study, (d) significance of the study, (e) definition of key terms, (f) assumptions, and (g) scope and delimitations.

\section{Background of the Study}

The United States is a nation with a long history in immigration. Immigration is the act of a person moving to a foreign country with the objective to reside there temporarily or permanently (Massey, 1995). Immigrants are people who, at birth, have no U.S. citizenship and include naturalized citizens, lawful permanent residents, temporary visa holders, asylum-seekers, refugees, and unauthorized individuals (Zong \& Batalova, 2017).

The first groups of immigrants to the United States were primarily western Europeans fleeing religious persecution (Massey, 1995). Along with the Native American Indians, the western European immigrants were the two ethnic groups of people initially on the land now considered the United States. They were joined by African Negro slaves imported for economic profit, as well as additional Europeans and Asian immigrants also in search of economic opportunities and religious freedom. Since then, people from a variety of ethnic groups around the world have become part of the American populace (Massey, 1995).

Individuals and people groups have crossed over land and sea to reach the United States for hundreds of years driven by extrinsic motivators that satisfy the most basic of human physiological and safety needs (Adler, 1977; Massey, 1995). According to Maslow's (1970) hierarchy of needs, physiological and safety needs include the need for 
food, water, shelter, physical safety, order, economic stability, and economic opportunities.

Many immigrants were looking for jobs in their new land driven by the hope of economic opportunities. By the turn of the 20th century, many immigrants found work in service sectors such as the hospitality and garment industries in cities all around America (Rischin, 1962). Jobs which required low technical skills such as production work in garment factories, and where the inability to speak English and understand the local culture had marginal impact on job performance, were highly sought after by immigrants (Rischin, 1962). Consequently, by 1914, the production of women's wear was the top industry in the state of New York (Rischin, 1962). Instances of immigrants using their pre-migration skills were also observed such as the matching of Jewish tailoring skills with the growing garment industry in New York (Bodnar, 1980). The U.S. garment industry provided jobs for immigrants as well as a pathway into a new culture, and meaningful economic opportunities.

Today, due to pervasive global conflicts, economic uncertainties, and environmental shocks, the face of immigration is rapidly changing. By the year 2015 , immigrants constituted approximately 14\% (43 million) of the 321 million people in the United States (Zong \& Batalova, 2017). A large percentage of these immigrants come from Mexico seeking economic opportunities. In fact, $27 \%$ of immigrants originate from Mexico, making Mexico the top source nation of people immigrating to the United States. India accounts for six percent of the U.S. immigrants, while China (including Hong Kong), and the Philippines rank third, each accounting for five percent of the U.S. immigrant population (Zong \& Batalova, 2017). 
United States immigration code defines a refugee as a foreigner of special humanitarian concern to the United States (U.S. Citizenship and Immigrant Services, 2015). A refugee is an individual who has been forced to leave his or her native country, is unable to return because of ongoing persecution due to race, religion, political opinions, nationality, and or membership to a social group that may lead to death (U.S. Department of Homeland Security, 2017). The United Nations High Commissioner for Refugees (UNHCR; The UN Refugee Agency, 2017) puts the total number of refugees worldwide at an all-time high of over twenty-two million people; and with the ongoing global conflicts, this number of refugees is expected to rise (2017a). According to the U.S. Department of State (2017), the number of refugees to be admitted to the United States annually from each region in the world is determined by the U.S. President and Congress. In 2015, 69,933 refugees arrived in the United States for resettlement (U.S. Department of State, 2017). Data from the U.S. State Department (2017) indicates that since 1975, more than three million refugees have been resettled in the United States. This quota is a great source of political tension and controversy between the United States and refugees' advocates because of the growing numbers of refugees who need resettlement but who have yet to be given that opportunity (Refugee Council USA, 2018). The refugee situation exerts significant pressure on the United States to accept and resettle refugees each year-a challenge the U.S. government is ill equipped to tackle.

Globally, humanitarian crises in the Middle East, Africa, South America, and Southeast Asia are changing the face of immigration as thousands of refugees are forced to leave their homelands in search of safe harbor motivated by the belief that they will be able to meet their physical, emotional, and social needs in neighboring countries 
throughout Europe and the United States (UNHCR, 2017). These ongoing waves of refugees to the United States continue to be influenced largely by refugees' expectations of a better life that is reinforced by recent history (Igielnik \& Krogstad, 2017). For example, during the 1990s, the United States successfully resettled many refugees who fled conflict in the former Soviet Union. Today, in order for refugees to be resettled in the United States, they must go through a process that includes: (a) referral to the U.S. Refugees Admissions Program for consideration; (b) multiple biographic and biometric security checks; (c) initial health assessments to ascertain if they are inadmissible to the United States on health-related grounds; and finally, (d) on admission, a final screening and assignment to a voluntary resettlement agency (VOLAGs) to resettle them (Pena, 1997). Most recently, the ongoing Syrian conflict has motivated many to flee their homes resulting in the resettlement of more Syrian refugees in the United States (Igielnik \& Krogstad, 2017). As global conflicts continue, the United States faces a moral and ethical dilemma as to whether they should accept more refugees over and above the annual quota established by the President and Congress (U.S. Department of State, 2017).

Regardless of what western country an immigrant chooses, the resettlement process is long and successful integration into the host country is difficult to guarantee (Refugee Council USA, 2018). Many host countries initially accept refugees but are unable to provide them with a permanent home and so, temporarily accommodate them in refugee camps with the mindset that the refugees will eventually return to their native countries. These camps or settlements offer limited educational and employment opportunities to the refugees that live in them (UNHCR, 2017). Many refugees are enticed by the promise of "the American Dream;" thus, they persevere through the 
process motivated by the expectation that once they arrive in the host country, and through hard work, they and their loved ones will soon be rewarded with the things they each define as pertinent to a better life (Sienkiewicz, Mauceri, Howell, \& Bibeau, 2013). Precedence reinforces this expectation and belief that if they can reach the United States, these refugees will be treated equitably like other refugees have been throughout history and, thus, they can expect to achieve physical, social, and economic stability (Adler, 1977). The reality is that the foreign policies of the United States and other western nations restrict the offer of permanent new homes to a limited number of refugees who cannot return safely to their own countries (UNHCR, 2017). In order to be resettled in the United States, a refugee must go through the process of applying and being accepted for resettlement through the UNHCR. The applications are then sent to the Department of Homeland Security's U.S. Citizenship and Immigration Services (USCIS). From there a determination is made as to whether the refugee applicant will be accepted in the country (U.S. Department of State, 2017). If the refugee applicant is accepted, a medical examination is performed, a U.S. location for resettlement is identified, and cultural orientation begins. After a year in the country, by law, refugees must apply for permanent residency. The process of resettlement can take up to 24 months (U.S. Department of State, 2017) and very few people grasp the full measure of the challenges refugees face when they flee their countries and even after they become permanent residents in the United States (Fangen, 2006).

The challenges experienced when refugees are resettled in the United States are driven by deficiencies in language, social networks, relevant and recognized formal education, technical skills, as well as limited understanding of, and sensitivity to, the new 
culture (Fangen, 2006). Many refugees struggle to make sense of their new environment (Sienkiewicz et al., 2013). Their struggle is exacerbated by the main tenet of U.S. refugee policy that states all refugees must become self-sufficient within 18 months of arrival to the United States (U.S. Department of State, 2017). To help address this deficit, nine nonprofit organizations (NPOs) involved in the U.S. refugee resettlement program continue the work to uncover what motivates refugees so they can be properly equipped to successfully resettle and be self-sufficient in this country (U.S. Department of Health \& Human Services, 2017). These organizations are called voluntary agencies (VOLAGs). They include Church World Service (CWS), Ethiopian Community Development Council (ECDU), Episcopal Migration Ministries (EMM), Hebrew Immigrant Aid Society (HIAS), International Rescue Committee (IRC), Lutheran Immigration and Refugee Service (LIRS), U.S. Committee for Refugees and Immigrants, U.S. Conference of Catholic Bishops/Migration and Refugee Services (USCCB), and the World Relief Corporation (WRC; U.S. Department of Health \& Human Services, 2017). These agencies partner with the U.S. Department of State through the Office of Refugee Resettlement (ORR) to provide resettlement services (Mott, 2010). The resettlement services help refugees learn English, obtain their Social Security cards, and secure employment (Mott, 2010). Despite the resettlement services available to refugees upon arrival to the United States, for many assimilation is a slow and arduous process (Tshabangu-Soko \& Caron, 2011). Adler (1977) also implied that some of the challenges refugees and immigrants alike go through are due to a state of impaired psychological functioning upon their arrival in the country of resettlement. It doesn't matter what level on Maslow's hierarchy of needs they had achieved pre-migration; the decision to flee 
one's native country introduces many negative factors that lead to a shift toward the bottom of the hierarchy making meeting physiological and security needs (something that was not even a consideration before) a high priority (Adler, 1977).

Assimilation that is an outcome of immigration occurs when refugees adopt the cultural traits of the dominant group, participate actively in the existing social institutions, and are accepted by the dominant group (Marger, 2006). Due to the ethnic, social, and religious diversity of the refugees currently being resettled in the United States, assimilation is increasingly difficult and no longer the inevitable outcome (Zhou, 1997). At the turn of the 20th century, European immigrants entering the United States had similar cultures and languages to the dominant group. Despite religious and ethnic differences, they were typically of Caucasian descent (Perez \& Hirschman, 2009). The Immigration and Nationality Act of 1965 abolished the quota system that was based on national origin, resulting in a significant number and rise in diversity of immigrants to the United States (Chishti, Hipsman, \& Ball, 2015). Some of the factors that play an important role in a refugee's ability to assimilate and thrive include access to economic opportunities, the opportunity for upward mobility, the strength of family ties in their native country, and the degree of cultural and religious similarities between the refugees and the dominant group (Zhou, 1997). Therefore, assimilation is not determined solely by the refugee but is largely influenced by efforts made by members of the host society (dominant group) to accept and integrate newcomers (Zhou, 1997).

As newcomers to the United States, refugees arrive with expectations of their new home country that are often idealistic (Sienkiewicz et al., 2013). This idealism is borne from stories of other immigrants who have resettled in the United States and have been 
able to achieve physical, social, and economic stability. The sense of expectancy is reinforced by the examples refugees cite within their extended families and communities and from biographies and news stories of other refugees who came to the United States with little to their name and, by applying themselves, achieved extraordinary success (Goldschein, 2012). This includes an individual like businessman Andrew Ly, one of the Vietnamese Boat People. The Vietnamese Boat People were economic and political refugees who fled Vietnam by boat and ship after the Vietnam War ended in 1975 (Goldschein, 2012). Ly was one of the Vietnamese Boat People who was resettled in the United States with the help of the U.S. Conference of Catholic Bishops/Migration and Refugee Services (USCCB) in the 1970s (Goldschein, 2012). Arriving with little to his name, and after a few years of working odd jobs, Ly learned the English language, pooled family resources together, and opened the Sugar Bowl Bakery. In 2012, his business was valued at $\$ 400$ million. Ly continues to be a visible beacon of success and was the recipient of a variety of awards that recognized his achievements and contributions to the United States (Goldschein, 2012).

Another notable example is Elie Wiesel. Born in Romania, author and activist Elie Wiesel was the only member his family to survive the Nazi concentration camps of Auschwitz-Birkenau (Goldschein, 2012). After World War II ended, Wiesel began writing, but it was not until he moved to the United States that he became published and one of the most prolific writers and public speakers on the Holocaust (Goldschein, 2012). Wiesel, like Ly, received much recognition for his work (Burke, 2016) and did much throughout his life to tell others, young and old alike, about the horrors of intolerance and to advocate for inclusion. 
The degree to which refugees experience some semblance of success that begins to mirror a pathway to the achievements of recent notable immigrants like Ly and Wiesel impacts how refugees settle into their new lives and integrate into society. For example, Fangen (2006) studied Somali refugees' ability to integrate into Norwegian society. Upon arrival in Norway, Somali refugees were enrolled in a Norwegian language course to help them communicate. Many Somalis were observant Muslims and thus required by their holy text (the Koran) to pray five times a day. Somali refugees expressed that the religious freedoms they had expected to find in Norway were being curtailed because they had to be in class during mandatory prayer times. They expressed a desire to stop attending the language course because it undermined their religious beliefs even though there was a monetary reward for course completion. These situations triggered feelings of humiliation among the refugees due to a mismatch in values and thus, expectations. The Somali refugees reported similar experiences in schools and places of employment where Norwegians failed to give them the freedom to pray during class or the workday, thus exemplifying how a mismatch between a refugee and a host country's expectations (value system) can impact a refugee's ability to successfully integrate into society. This is an example of expectancy theory in action- the Somalis had high levels of confidence that if they applied themselves to the course (instrumentality), they would gain language proficiency and receive the monetary reward (valence) (Vroom, 1964). Unfortunately, the Norwegians, though well intentioned, didn't do enough to understand an important extrinsic/hygiene factor-the time to pray, and to design a language program that accommodated this need. The value of the monetary reward was insignificant in 
overcoming intrinsic rewards derived from practicing religion and maintaining relationships within the Muslim community (Fangen, 2006).

In another study, Howard, Nazari, and De Leal (2006) interviewed Afghan refugees resettled in St. Louis, Missouri and found that upon arrival in the United States, these refugees expected to receive free healthcare similar to what is available in other countries such as Canada and the United Kingdom. Additionally, they hoped to quickly secure meaningful employment (Howard, Nazari, \& De Leal, 2006). The Afghan refugees quickly learned that in the United States, access to healthcare is possible if an individual has medical insurance (often offered as a benefit by employers) or the ability to pay out-of-pocket to see a medical practitioner. Some refugees also expected to receive disability benefits when they resettled to the United States because of pre-existing chronic conditions or due to advanced age (Howard et al., 2006). However, many did not qualify for benefits under the Americans with Disabilities Act (Howard et al., 2006). When it came to pre-migration expectations related to securing a meaningful job, refugees expected to quickly find employment in the area of their expertise. By finding fulfilling and well-paying jobs, refugees believed they would be better off financially and thus able to purchase a home and meet all the needs of their families (Howard et al., 2006). More often than not, refugees were disappointed with the reality of their professional lives. Most refugees were unable to find jobs or, at best, secured jobs for which they were overqualified and grossly underpaid. In reality, many ended up in cramped living quarters, underemployed, and challenged to support their families (Howard et al., 2006). 
Using data from the American Community Survey (ACS) between the years of 2009-2011, the U.S. Census Bureau (DeNavas-Walt \& Protor, 2014) approximated that $44 \%$ of refugees, and $43 \%$ of non-refugee immigrants in the United States were lowincome, earning an income that was $200 \%$ below the federal poverty level (Capps \& Newland, 2015). The federal poverty level (FPL) is an income measure provided each year by the U.S. Department of Health and Human Services to determine an individual's eligibility for specific state and federal benefits and programs (U.S. Department of Health \& Humans Services, 2018). However, the census data also showed that the share of lowincome refugees living well below the federal poverty line declined as the number of years living in the United States increased, reducing a refugee's reliance on federal programs (Capps \& Newland, 2015). These statistics not only reflect the economic hardship many refugees face, but the fact that their conditions can, and often improve over time (Becker \& Isaacs, 1996).

A significant factor contributing to successful integration and resettlement is the refugee's ability to secure meaningful employment that provides stability and a living wage (Olliff, 2010). This is why programs that help refugees secure fulfilling employment are so vital. Employment is particularly important to refugees as it socializes them to acceptable work practices, contributes to their cultural integration, links them to conventional institutions, and provides income that facilitates their economic selfsufficiency (Berger, 2004). Adult women with refugee backgrounds were the targeted participants of this research study because empirical research indicated that gender shapes the experiences of many refugees in America (Suarez-Orozco, 2013). Many obstacles to employment exist for women with refugee backgrounds, namely, education, 
English language proficiency and lack of support with childcare (Berger, 2004; Yashuko, 2006). Women with refugee backgrounds are twice more likely than their male counterparts to be widowed, divorced, or separated and more likely to bear the financial and caretaking responsibility for children (Suarez-Orozco, 2013). Without access to employment training and opportunities during the early resettlement phase, refugees lack the income and resources to be economically self-sufficient, which can increase their risk of social and labor exclusion.

There are four key factors associated with many refugees' inability to secure employment. These include: (a) lack of critical networks such as family and friends in employment, (b) limited vocational skills relevant to the labor market, (c) limited relevant work experience prior to arrival, (d) and lack of familiarity with the overall system (Victorian Settlement Planning Committee, 2008). Due to limited resources and overwhelming demand, resettlement agencies can only offer refugees limited support in the areas of employment and career/vocational counseling and continuing education that develops relevant vocational and language skills. As a result of this ongoing unmet need, organizations such as textile and apparel NPOs have stepped in to assist with settlement and integration efforts (Keiler, 2017; Saez, 2017; Bowen, 2016; Nielsen, 2015)

Textile and apparel NPOs are organizations within the U.S. apparel industry that are classified under the nonprofit classification scheme. These organizations are missioncentered (Boyd et al., 2009). Some NPOs have found success in recruiting, training, and hiring marginalized populations (e.g., high school dropouts, ex-offenders, recovering addicts, refugees) to address shortfalls in affordable staffing that enable the organizations to operate profitably in an increasingly global and competitive environment (Reddy, 
2014). Prior textiles and apparel researchers have addressed issues of social responsibility such as fair trade (Goworek, 2011; Littrell \& Dickson, 1997) and environmental sustainability (Goworek, 2011), but neglected the role of textile and apparel NPOs in the training of individuals with refugee backgrounds for the purpose of vocational rehabilitation.

Much of the literature on co-operatives, charities, and NPOs in the textile and apparel industry has been conducted outside the United States and has focused on the products made and processes used to market products to the United States or other developed countries (Hassanin, 2008; Page-Reeves, 1998; Littrell \& Dickson, 1997). To date, none of these studies have focused on what motivates marginalized trainees or workers, in particular those of refugee background, to successfully integrate into the U.S. apparel industry. Historically, employment in apparel production was one of the primary avenues for meaningful employment opportunity for immigrants (Goldin \& Sokoloff, 1982). Thus, the role of textile and apparel NPOs in the economic advancement of people with refugee backgrounds is relevant to today's discussion of refugee resettlement and their successful integration into U.S. communities.

In an effort to identify what contributes to the attainment of training outcomes for women of refugee backgrounds in the United States that could lead to the potential successful integration of these individuals into the U.S. workforce, this study explored the career transition experiences of women with refugee backgrounds in multiple textile and apparel NPOs in the United States to better understand how training shaped those experiences. The results pointed to ways training programs could be improved with the removal of identified barriers identified by the women interviewed. Removal of such 
barriers may lead to the attainment of training outcomes by more training program participants as obstacles are identified and removed.

\section{Statement of the Problem}

Economic integration is the process of obtaining work and becoming a part of the employed workforce in a particular society (Bulcha, 1988). To be considered economically integrated into a given society, individuals with refugee backgrounds need to have reached a level of self-sufficiency (Bulcha, 1988). They may have left meaningful employment in their native countries before being resettled in the United States. Though motivated to integrate and provide their families with economic stability, individuals of refugee backgrounds face a plethora of challenges. Some of the identified challenges include low motivation and self-efficacy, human agency, difficulty in identifying and taking advantage of professional development opportunities, and conflicting family needs (Maynard \& Ferdman, 2009). Motivation has been identified as an especially important component to the productivity of trainees and employees alike, and so, the retention and promotion of workers and trainees in an organization are dependent on motivation (Ankli \& Palliam, 2012).

Highly motivated trainees and workers report overall higher levels of satisfaction and self-esteem at work (Ankli \& Palliam, 2012). Many refugees are motivated to immigrate to the United States, expecting a better life where they believe they will experience physical, religious, political, and economic security (Howard et al., 2006). However, many of them arrive in the country with few relevant skills, credentials that are not recognized, and nominal social networks. Regardless of whether their skills and education match the needs of the U.S. labor market, it is imperative that refugees quickly 
secure meaningful and sustainable employment that leads to economic self-sufficiency as soon as possible (U.S. Department of Health \& Human Services, 2017).

\section{Purpose of the Study}

The purpose of this study was to gain in-depth understanding of the career transition experiences of women with refugee backgrounds within the context of textile and apparel NPOs in the United States by exploring what motivated them to participate in the training programs and attain training outcomes. An embedded multiple case study approach was used to study graduate and current trainees participating in textile and apparel NPOs' formal skills training programs; training providers in each NPO knowledgeable about the organization's structure, vision, and mission were also interviewed. The research sites were limited to textile and apparel NPOs located in a large urban area in a state in the Northwest region of the United States.

To accomplish this purpose, the research questions are:

Research Question 1: What facilitates the motivation of women trainees of refugee backgrounds to participate in career transition programs and attain training outcomes?

Research Question 2: What hinders the motivation of women trainees of refugee backgrounds to participate in career transition programs and not attain training outcomes?

In this study, a career transition was defined as participation in a formal training program (Liptak, 2008).

\section{Significance of the Study}

The findings of this study benefit the textile and apparel field in three distinct ways: (a) the findings generated new insights on barriers and disincentives that contribute 
to the body of knowledge around the needs and experiences of women trainees of refugee backgrounds participating in textile and apparel NPOs' training programs; (b) the findings contributed insight to textile and apparel NPOs that influence the recruitment strategies of future trainees that optimize expectancy, instrumentality and valence; and (c) the findings contributed to the study of textile and apparel NPOs in textile and apparel programs at colleges and universities as alternate business models that fulfil a unique need in the market.

The findings of this study benefit government agencies and humanitarian organizations that respond to global refugee crises in three distinct ways: (a) they justified the need for formal K-12 education and employment opportunities for refugees living in camps, (b) they validated the importance of English language classes for refugees living in camps, and (c) they defended the reason why there should be support for refugees participating in vocational training programs in camps, to enable them to find work opportunities, and change learned dependency behaviors that are a consequence of living in refugee camps and being dependent on rations. Exposure to work will help refugees develop views and habits about work and life that are more similar to ordinary life, and thus, when resettlement occurs, adjustment may not be as difficult.

The study derived a deeper understanding of congruency between motivations, expectancy, and outcomes experienced by women of refugee backgrounds participating in training programs in a textile and apparel NPO within the context of expectancy theory (Vroom, 1964). Recommendations were noted on how textile and apparel NPOs may optimize their programs and services to create symbiotic systems of rewards for their 
stakeholders (refugees, local business partners, and their own mission minded staff) and consequently positively influence the rate of successful integration of female refugees into the U.S. workforce and, ultimately, American society.

\section{Definition of Key Terms}

The definitions of key terms used throughout the text are provided below:

Assimilation: Assimilation is an immigration outcome that is the result of a complex process by which immigrants adopt the cultural traits of the dominant group, participate actively in the existing social institutions, and are accepted by the dominant group (Akresh, Massey, \& Frank, 2014).

Career Transition: Career transition occurs through participation in a formal training program (Liptak, 2008).

Expectancy: Expectancy is an individual's belief that an increase in work effort would be followed by an increase in their performance (Dinibutun, 2012).

Extrinsic motivation: Extrinsic motivation is defined as behaviors that are carried out because they lead to a separable outcome such as reward systems, grades, or payment (Ryan \& Deci, 2000).

Instrumentality: The perception by an individual that a given outcome of performance will result in an anticipated reward (Vroom, 1964).

Intrinsic motivation: Intrinsic motivation is defined as behaviors that are carried out of self-interest of enjoyment that can help sustain passion, creativity, and effort (Ryan \& Deci, 2000).

Integration: Integration is "a situation in which host and refugee communities are able to co-exist, sharing the same resources, both economic and social, with no 
greater mutual conflict than that which exists within the host community" (Harrell-Bond, 1986, p. 7).

Low-income: Low-income refers to individuals with an annual family income below 200\% of the federal poverty level (FPL) (Capps \& Newland, 2015).

Marginalization: Marginalization "typically involves some degree of exclusion from access to power and or resources. In being at the margins of society, those who are marginalized do not get to enjoy the full or typical benefits that those who are closer to the center tend to receive" (Maynard \& Ferdman, 2009, p. 25).

Motivation: Motivation is an internal state that serves to stimulate behavior and give it direction (P. Kleinginna \& Kleinginna, 1981).

Need: A need is a condition within an individual that makes given results appealing (Ramlall, 2004).

Pre-training Motivation: Pre-training motivation is the intended effort towards mastering the content of a training program (Cheng \& Ho, 2001).

Refugee: A refugee is an individual who has been forced to leave his or her native country and is unable to return because of persecution or fear of death because of race, religion, or political opinions, and officially resettled in the United States as a refugee (UNHCR, 2017).

Resettlement: With oversight by the UNHCR, refugees from a country of asylum are moved to another country that has given them admission, and where the refugees will eventually assume permanent residency (UNHCR, 2017).

Resiliency: Resiliency is linked to an individual's capability to overcome and cope with challenges and adversity (Wagnild \& Collins, 2009). 
Self-efficacy: Self-efficacy is an individual's belief or confidence in their ability to exercise some measure of control over their own motivation, behavior, and social environment in the face of stressful events to achieve a specific goal (Bandura, 1997, p 20).

Training: Training is the systematic development of the knowledge, skills, and expertise required by a person to effectively perform a given task or job (Patrick, 2000).

Textile and apparel NPO: The textile and apparel NPO is a philanthropic organization founded to serve an unmet need in communities through the provision of programming and services, such as training programs focused on the skill development of workers to produce textile-based products (Ingram, 2013).

Training outcomes: Training that is focused on the exposure, immersion or mastery of knowledge, skills and information that relates to a particular profession or industry (Charles, Bainbridge, \& Gilbert, 2004).

Training performance: Training performance as an outcome is the degree to which the individual believes that performing at a particular level will lead to the attainment of a desired result (Vroom, 1995).

Training providers: Training providers include the instructors, directors, program coordinators, teaching assistants, lead artisans, community outreach personnel, and founders in textile and apparel NPOs who support the delivery of training programs to women with refugee backgrounds.

Training Motivation: Training motivation is the direction, intensity, and persistence of learning-directed behavior in a training environment (Kanfer, 1991). 
Valence: The degree to which a person has a preference for a given outcome, or the anticipated satisfaction a person believes they will derive from a given outcome (Vroom, 1964).

Value: The actual satisfaction that a given outcome provides (Vroom, 1964).

\section{Assumptions}

For the purposes of this study, it was assumed that women trainees with refugee backgrounds willingly participated in the career transition program and, in doing so, were able to, and willing to, work on building or enhancing their sewing skills. It was also assumed that these participants provided truthful and insightful responses and did not feel threatened or intimidated into disclosing information during the interview process, regardless of whether their training experience was positive or negative. To enforce this assumption, participant privacy protection measures were used.

With regard to the career transition programs selected for this study, it was assumed that these programs were representative of other career transition programs offered to women of refugee backgrounds in terms of training outcomes that were referenced in the literature review (Renner \& Senft, 2013), and were organized in ways that provided trainees with learning opportunities that progressively enhanced their sewing skills. A careful review of the mission statements and the general program curriculum of each of the career transition programs represented in this study affirmed these assumptions.

It was also assumed that each training provider that instructed and or mentored the trainees supported their skill development and provided opportunities for trainees to apply their skills during the program and upon successful completion of it. Additionally, 
it was assumed that training providers valued and encouraged the applied use of sewing skills acquired throughout the course of the career transition program and thereafter.

\section{Scope and Delimitations}

One of the functions of NPOs is to support community building activities that nurture connections among people (Cameron, 2004). In this study, the textile and apparel NPOs in a large U.S urban area offered career transition programs that assisted people with refugee backgrounds to not only learn or build on their sewing skills, but to also start developing a social network that can lead to job opportunities and eventually better integration into the communities in which they live. The purpose of this study was to gain in-depth understanding of the career transition experiences of women with refugee backgrounds within the context of textile and apparel NPOs in the United States by exploring what motivated them to participate in the training programs and attain training outcomes. Current and graduate trainees of career transition programs offered by textile and apparel NPOs were chosen because this allowed for the study of what hinders and facilitates women's motivation to participate and attain training outcomes to be investigated from two perspectives, each one with valuable insights. The length of time individual trainees had spent in the career transition program or the time that lapsed since their successful completion of the program was not tracked.

This study had a sample of 24 participants comprised of graduate trainees, current trainees, and training providers affiliated with career transition programs in five textile and apparel NPOs. To constrain the scope of the research study, the research questions were framed by participants' depth of motivation and personal needs for training outcomes while training and after completion of the program. The study did not include 
former trainees of the career transition programs who had dropped out before completion because the focus was on participants who were making strides towards successfully achieving training outcomes. Each participant was interviewed about their individual career training experiences at their textile and apparel NPO. For participants who had already graduated from the training programs, the researcher visited the specific training sites and used an observational protocol to assess the environment in which the training had occurred, and interviewed the training providers, but without directly observing a training class in progress.

\section{Organization of the Study}

This dissertation is comprised of five chapters. Chapter I consists of the background of the study, statement of the problem, purpose of the study, significance of the study, definition of key terms, researcher assumptions, scope and delimitations, and organization of the study. Chapter II presents a literature reviews on career transition and training, refugee resettlement, strategies used to increase refugee employability, theoretical framework, and the research gap. Chapter III discusses the researcher's philosophy, research methodology and design appropriateness, elaboration and rational for research design, the role of the researcher, research questions, sample size, instrumentation, informed consent, protocol, recordings and coding, data collection timeframe, and analysis. Chapter IV presents a description of the sample, thematic categories and interpretations, a conceptual model, and researcher's reflection. In conclusion, Chapter V contains an overview of the study, contributions and implications for theory, as well as practice, and limitations and recommendations for future study. Following Chapter V are the References and Appendices. 


\section{CHAPTER II: LITERATURE REVIEW}

Chapter II contains the following sections: (a) Career transition and training, (b) refugee resettlement, (c) strategies used to increase refugee employability, (d) theoretical framework, and (e) research gap.

Using an inductive approach, the purpose of this study was to gain in-depth understanding of the career transition experiences of women with refugee backgrounds within the context of textile and apparel NPOs in the United States by exploring what motivated them to participate in the training programs and attain training outcomes. A multiple embedded case study approach was used to study the graduate and current trainees participating in textile and apparel NPO's formal skills training programs. Training providers in each NPO knowledgeable about the organization's structure, vision, and mission were also interviewed. To accomplish this purpose, the central research question was: What facilitates or hinders the motivation of women trainees of refugee backgrounds to participate in career transition programs and attain training outcomes? In this chapter, literature, concepts, and theories that are relevant to this study are presented.

\section{Career Transition and Training}

A transition is "an event or nonevent that results in a change in assumptions about oneself and the world and thus requires a corresponding change in one's behavior and relationships" (Schlossberg, 1981, p. 5). Transitions in values, personal philosophy, and personal appearance are part of the human experience. To attain certain goals, especially career goals, people often have to go through several transitions throughout their lifetime. Career transitions include events such as advancing from school to work, workplaces being downsized, changing jobs or career paths, or experiencing changes in required work skills and or schedules (Liptak, 2008). Regardless of how individuals prepare, some 
of these transitions are difficult to navigate due to numerous factors that an individual may or may not be able to control (Liptak, 2008). For the purpose of this study, career transitions pertained to the participation by women of refugee backgrounds in sewing skills training programs offered by a textile and apparel NPO with the expectation that completion of the training would lead to meaningful and sustainable employment and careers in the apparel industry.

The topic of training has been the subject of scholarly research for at least 30 years. Salas and Cannon-Bowers (2001) noted that over time, the focus of training research has shifted to concentrate on a systems approach as the concern became centered on the organizational context in which training occurs. Previous empirical studies regarding training revealed that certain pre-training conditions are as important to individual training performance and outcomes as those that occur during the training program, and once the training is completed (Shute \& Gawlick, 1995; Quinones, 1997). Researchers illustrated how pre-training activities influence the effectiveness of training (Alvarez, Salas, \& Garofano, 2004; Tannenbaum, Cannon-Bowers, \& Mathieu, 1993). Important factors include: (a) what a trainee brings to the training environment, (b) how engagement and participation is promoted in training activities, and (c) how training is delivered so as to maximize learning (Alvarez et al., 2004; Tannenbaum et al., 1993). Each one of these factors is further explained below.

Individual characteristics that impact the effectiveness of training. The importance of what a trainee brings to the training environment is discussion-worthy because individual characteristics such as intellect, self-efficacy, and goal orientation have been shown to impact the effectiveness of training (Alvarez et al., 2004; 
Tannenbaum et al., 1993). Those trainees who have better than average levels of intellect show a propensity to thrive within the training context (Ree, Carretta, \& Teachout, 1995). Ability directly impacts the attainment of job knowledge (Ree et al., 1995). When a trainee has a high level of intellect, self-efficacy and performance are elevated, ultimately helping with skill acquisition (Colquitt, LePine, \& Noe, 2000; Salas \& Cannon-Bowers, 2001; Warr \& Bunce, 1995). Salas and Cannon-Bowers (2001) suggested that more studies needed to focus on trainees who may demonstrate low intellect or low educational levels, so that effective training methods can be developed for this population. While cognitive ability may be an effective predictor of successful performance in training contexts, it does not automatically translate to job performance. Jobs may have requirements that require other abilities such as psychomotor skills such as dexterity, coordination, precision, and control for success (Salas \& Cannon-Bowers, 2001). A critical factor in determining whether cognitive ability or intellect will be important to job success is understanding the nature of the job (Salas \& Cannon-Bowers, 2001).

Another individual characteristic influencing the acquisition of training is selfefficacy. Self-efficacy was first defined in Bandura's (1986) social cognitive theory, and refers to an individual's ability to accomplish a task or control their behavior, motivations, or environment. Self-efficacy beliefs have been described as “people’s judgments of their capabilities to organize and execute courses of action required to attain a desired outcome" (Bandura, 1977a, p. 3). Personal self-efficacy beliefs are important to career development (Betz, Klein, \& Taylor, 1996). Researchers of training and education have irrevocably proven that a trainee with self-efficacy will have better learning and performance outcomes than one who does not (Phillips \& Gulley, 1997). The belief that 
one can perform specific tasks and behaviors has also been shown to influence other personal attributes, namely training and adjustment in newcomers, as well as job satisfaction (Saks, 1995).

Goal orientation is categorized as a mental framework that individuals use to interpret and behave during learning-oriented activities (Salas \& Cannon-Bowers, 2001). Two classes of goal orientation have been identified in the training literature: (a) mastery goal orientation, and (b) performance goal orientation. According to Dweck (1986), mastery goal orientation is when an individual seeks to become proficient by learning new skills and mastering new situations. Performance goal orientation occurs when an individual actively seeks out positive performance evaluations and avoids negative ones in order affirm their proficiency (Dweck, 1986). Both classes of goal orientation were relevant to this research study because each trainee in a career transition is motivated to participate in a training program with a goal in mind. Training providers expect trainees to aspire toward mastery of new skills learned and to actively seek out ways to affirm their newly acquired skills and knowledge, thus bringing alignment between trainee goals and training outcomes (Salas \& Cannon-Bowers, 2001).

Motivations to participate in training. How a trainee's engagement and participation is promoted during training activities will have a bearing on the effectiveness of training. Once in a training program, a trainee's motivation to learn and attend training will impact the trainee's skill acquisition, retention, and willingness to apply the newly acquired knowledge and skills in a future work situation (Salas \& Cannon-Bowers, 2001). Training motivation is defined as the direction or persistence in effort that a trainee applies to learning-oriented activities before, during, and after 
training (Tannenbaum \& Yukl, 1992). Colquitt, LePine, and Noe (2000) suggested that training is complex and subject to individual and situational characteristics. Individual characteristics include intelligence, self-efficacy, diligence, apprehension, and age. Situational characteristics refer directly to the training environment. Colquitt et al. found that older workers displayed lower motivation, learning, and post-training efficacy. Their findings showed a connection between age and motivation to learn that warrants more investigation, especially given that refugee women seeking skills training are typically mature women with low educational attainment and little experience with technologydriven instruction (Colquitt et al., 2000). New designs of training environments are needed to accommodate refugee women's needs. A deeper understanding of training motivation is important for learning and has direct implications for the design and delivery of training (Salas \& Cannon-Bowers, 2001).

Instructional methods for training. The way that training is delivered will determine if learning is maximized (Kirlik, Fisk, Walker, \& Rothrock, 1998). There are many different instructional methods used during training; the most effective ones take into consideration these four factors: (a) presentation of pertinent information, (b) demonstration of knowledge and skills that need to be learned, (c) creation of opportunities for skill practice, and (d) provision of feedback during and after the training exercises (Salas \& Cannon-Bowers, 2001). To begin with, trainees are given relevant information early in the training process to adequately prepare them for applicable training exercises (Johnston \& Cannon-Bowers, 1996). Then, a show of applicable knowledge and skills is made in small groups versus individually to reduce the amount of interaction anxiety and to support vicarious learning and peer-to-peer interaction among 
trainees (Arthur et al., 1997). Finally, trainees are given an opportunity to apply and practice their newly acquired skills in an environment that closely reflects a real work environment (Johnston \& Cannon-Bowers, 1996). Feedback from training providers is supplied throughout the training exercises as well as upon completion (Salas \& CannonBowers, 2001).

Post-training events are just as significant as those that transpire beforehand and throughout training, but much of the research on what happens after training has concentrated on training program evaluation (Salas \& Cannon-Bowers, 2001). The design and delivery of training programs were evaluated, and while results attested to the value of training, more research is needed on failed programs (Salas \& Cannon-Bowers, 2001). Post-training events such as the extent to which the knowledge and skills acquired in the training program are utilized in the workplace, are important to ascertain the effectiveness of training (Salas \& Cannon-Bowers, 2001).

Training mature adults is different from training their younger counterparts, yet younger adults have been the focus of studies examining traditional learning strategies in career transition programs (Taylor \& Giannantonio, 1990). Mature adults approach learning with their prior knowledge and experiences which are foundational to their learning experiences and act as reservoirs of information (Knowles, 1990). Knowles (1984) made other assumptions about the adult learners that included: (a) adult learners' readiness to learn is connected to the developmental task of their social roles, (b) an adult learner is more oriented to apply the knowledge and skills they have learned immediately versus postponing it, and (c) an adult leaner is internally motivated to learn. Knowledge and experience help the adult trainee understand what is being learned (Plimmer \& 
Schmidt, 2007). At first, adult trainees may feel like imposters as many of their assumptions, beliefs, and expectations are threatened (Brookfield, 1999). Findings from adult education studies report that many adult learners have a sense of limited opportunity as family obligations restrict their ability to participate in educational opportunities (Zunker, 1990). However, adult learning is gaining increasing importance primarily due to two current societal developments: the retraining of an aging workforce, and the training of resettled refugees without transferable job skills (Workforce Training \& Education Coordinating Board, 2008). This research was concerned with the latter of those developments; therefore, the remainder of this literature review will focus on the training of resettled refugees.

\section{Refugee Resettlement}

The arrival of large numbers of refugees across Europe and North America suggests a changing workforce in developed countries, underlining the topic of refugee resettlement (Champion, 2018). Work and English language proficiency are central to a successful resettlement experience; the earliest studies on refugee resettlement in the United States focused on the experiences of Cuban and Vietnamese refugee groups in career transition programs (Holloway, 1976; Portes, 1969). By all indications, these refugees experienced downward occupational mobility upon arrival in the United States, but the one factor that was pivotal to transitioning the refugee into mainstream society was employment (Stein, 1979; Taft, North, \& Ford, 1979). Through employment, refugees established a social network, expanded and practiced their English language skills, discovered and conformed to group norms that led to their social acceptance by the dominant group (Stein, 1979; Taft et al., 1979). More importantly, employment provided 
income that led to a key tenet of U.S. refugee resettlement policy -self-sufficiency. Early researchers of these groups acknowledged the importance of occupational assimilation to their successful integration; a refugee who was working and self-sufficient was likely to have fewer problems adjusting to their new life versus one who remained isolated at home and or relied on the social safety net for extended periods of time (Finnan, 1981; Portes, 1969). Similar findings were described by Ward, Bochner, and Furnham (2006) who pointed out that psychological symptoms, especially posttraumatic stress, are exacerbated by unemployment, and conversely end up diminishing a refugee's chances of finding work.

To support the resettlement of these new groups of refugees arriving on U.S. soil, President Kennedy initiated one of the first federal programs by way of federal aid in the form of student loans, and funding for English language programs, vocational training programs, and professional retraining programs (Holloway, 1976). Cubans fleeing Cuba for Florida after the rise of the Castro regime, unlike their WWII predecessors primarily from eastern Europe, were greater in number, well-educated, and had held professional occupations in Cuba (Holloway, 1976). However once in the United States, these refugees faced struggles common to newly resettled refugees: (a) lack of English language proficiency, (b) age, (c) non-transferable skills and credentials, and (d) a lack of income (Holloway, 1976). Yet many of them survived the adversity of their early resettlement experiences due to family support and an emphasis placed on education, work and achievement, and later moved on to satisfactory occupations (Portes, 1969). The federally-sponsored education and work programs helped many Cubans refugees successfully integrate into U.S. society (Holloway, 1976). Programs such as a three- 
month course developed to assist refugees who had been doctors in Cuba, focused on English language medical terminology and trained Cuban doctors in taking English language exams (Holloway, 1976). The intended program outcome was for Cuban doctors to return to their original occupations and not experience permanent downward occupational mobility. These courses were later expanded to accommodate other graduates from foreign medical schools; soon, Vietnamese refugees resettled in Florida also enrolled in them (Holloway, 1976).

Vietnamese refugees, like their Cuban counterparts, ultimately assimilated well to life in the United States (Portes, 1969). Both groups were originally political refugees from middle/upper class families in their native countries. The Vietnamese refugees were educated, although not in the professional fields that their Cuban counterparts were; theirs had been a rigorous high school education that incorporated basic electronics theory into math and science curriculum (Finnan, 1981). It is no wonder that many Vietnamese refugees gravitated toward a career transition program that would gain them entry into the burgeoning electronics industry in the 1970s and 1980s. Within the context of a six-month electronics technician training program, Finnan (1981) conducted an ethnography on the process of career training Vietnamese refugees underwent to enter new occupations in the electronics industry. The researcher embedded herself alongside the Vietnamese trainees, and through participant observations, interviews with trainees at the current training facility and other training facilities, and interviews with Vietnamese refugees working as technicians, discovered a strong support network among the trainees that motivated each trainee to participate in the training program. That decision was later reinforced by other Vietnamese refugees already employed in the U.S. electronics 
industry that helped trainees not only choose the training program to enroll in, but sometimes even find employment in the industry as well (Finnan, 1981). This finding supports the assertions that have been made by researchers on collectivism; people from collectivist societies such as the one the Vietnamese trainees were from, depend on the opinions and support from family and friends and get fulfillment from achieving group objectives (Martella \& Maass, 2000). The new occupations also helped refugees recapture their self-worth and focus on the present leaving their struggles to a distant memory.

The Cuban and Vietnamese refugee groups bore similar characteristics to each other; they were well-educated, middle/upper class and lacked English language proficiency on arrival to the United States, but were more likely to assimilate successfully than their working class counterparts (Portes, 1969). Although Finnan (1981) confirms that refugee groups with middle/upper class backgrounds have to adjust to a lower occupational status than they held in their home countries, the refugees appeared satisfied with their new occupations because within their ethnic communities, occupational roles were redefined and the occupational hierarchy was recreated differently than it was in their native countries (Finnan, 1981).

These findings highlight the barriers to integration faced by the early refugee groups on arrival in the United States. They also point to the important role the receiving community played in helping the newly resettled refugees integrate, particularly in offering vocational training and professional retraining programs (Portes, 1969; Holloway, 1976). It is evident that both groups of refugees suffered downward occupational mobility upon arrival in the United States (Finnan, 1981). However, once 
enrolled in these training programs, Cuban and Vietnamese refugees were able to learn the English language and train or retrain in the vocation or field of their choosing before securing satisfactory employment (Holloway, 1976). Another reported outcome of employment was that refugees experienced fewer mental health problems (Holloway, 1976). However, neither study focused particularly on women refugees' access to or experience with the training programs. Furthermore, neither of these studies explored any of the factors that may have hindered refugees in participating and attaining the training outcomes of the programs, such as collectivist attitudes that apparently alleviate the psychological consequences of being unemployed (Martella \& Maass, 2000), and decrease the willingness to relocate for a job (Otto \& Dalbert, 2012).

Subsequent empirical studies on the subject of training programs within the refugee resettlement domain focused squarely on English language skill acquisition (Alatis, 1979; Deem \& Marshall, 1980; Freire, 1990). Although that topic is outside the scope of this study, it is necessary to mention that findings from these studies indicated, among other things, that language proficiency, being well-educated, perceived social support, and being younger in age increases refugees' chances of occupational integration, demonstrating their importance to career development (Ward, Bochner, \& Furnham, 2006).

Factors affecting refugee training participation and outcomes. Refugees face many barriers when they endeavor to participate in training programs. Many of the individual characteristics mentioned in the training and adult education literature are pertinent to a discussion of training outcomes. Systemic and pervasive gender beliefs (Lipsky \& Nimol, 1993), ethnic and religious differences (Casimiro, Hancock, \& 
Northcote, 2007; Fozdar, 2012), language barriers (Bloch, 2004; Valtonen, 2004), discrimination (Correa-Velez, Gifford, \& Barnett, 2010), underemployment (Goodman, Vesely, Letiecq, \& Cleavland, 2017), and the lack of recognition by employers of preexisting academic and professional credentials and professional work experiences from their native countries (Kogan, 2003; Bakker, Dagevos \& Engbersen, 2016) are some of the issues refugees encounter when exploring employment opportunities. Finn (2010) also cited that in many instances, the trauma experienced by refugees through violence, assaults, and persecution, or threats of such adversities that forced them to flee their native countries, either interrupted their formal education or was the reason they never received one. Therefore, lack of, or very little, formal education is included in the factors that affect training outcomes (Finn, 2010). These factors have been found to have the undesirable effect of diminishing the rate and likelihood of successful assimilation (Bloch 2002; Colic-Peisker \& Tilbury, 2006; Tomlinson, 2010), and lead to downward occupational mobility of refugees in the country of resettlement (Krahn et al., 2000).

\section{Characteristics of female refugees in the United States}

Currently, 21.1 million immigrants live in the United States; refugee women make up 51\% of this foreign-born population (Migration Policy Institute, 2015). Gender shapes the experience of most immigrants who arrive in the United States. Women are particularly disadvantaged, with female immigrants being twice as likely as their male counterparts to be widowed, divorced, or separated from their spouses (Migration Policy Institute, 2015). Suarez-Orozco (2013) found that women are also more likely to bear the financial and caretaking burden for dependents (e.g., children and elderly parents) in their new countries and therefore, are especially vulnerable to systemic forms of employment 
marginalization and discrimination. Tomlinson (2010) reported similar findings in her study of refugee women from African, Eastern European, and Asian countries living in the United Kingdom. She learned that although the women had previously held diverse occupations in their own countries ranging from dress makers to engineers, they had been unsuccessful in reestablishing themselves in the United Kingdom (Tomlinson, 2010). One of the many factors contributing to this disparity was the women's sole responsibility for caring for dependents such as children and aging parents/relatives. The inability to secure safe, reliable, and affordable dependent care made it difficult for the women to participate in available training programs such as English language courses (Tomlinson, 2010). Watkins, Razee, and Richters (2012) conducted an ethnography of 10 Burmese women resettled in Australia. The researchers pointed to not only gendered, but also cultural and socio-political factors during the entire immigration process that constituted barriers to educational attainment and access to the labor market for the Burmese refugees (Watkins, Razee, \& Richters, 2012). Women and girls who were unable to receive formal education due to cultural norms in their native country were restricted to the home, and as a result, were pre-literate in their native language. Upon resettlement in Australia, the responsibilities of child rearing, caretaking of elderly parents and in-laws, and general household management was the sole responsibility of the women, leaving them with limited opportunities to take advantage of the free community-based English language classes offered in their host country (Watkins et al., 2012). Thus, as women remain in their traditional roles as caregivers, they suffer from social isolation (Bloch, Galvin, \& Harrell-Bond, 2000). Those Burmese women who enrolled in the classes expressed a need for more English language classes (Watkins et 
al., 2012). Due to cultural conditioning, the women did not openly voice their dissatisfaction or complain about unmet needs unless probed directly (Watkins et al., 2012). The resulting achievement gap in English language proficiency of refugee women versus refugee men was evident in Watkins et al.'s study. This study revealed the important role gender plays in the ability for refugee women to access learning opportunities that facilitate the integration process in their countries of resettlement, and perhaps the need for a more nuanced approach to developing educational programs that take into account differing cultural and gender norms. Without such opportunities, refugee men will continue to take advantage of training openings. Consequently, more research is needed on the experiences of women refugees in refugee resettlement and integration programs in the United States.

Poor knowledge of the language spoken in the country of resettlement and a lack of formal qualifications may be early predictors of unemployment among refugees (Kogan, 2003). English as a second language classes traditionally assist newly-arrived adult refugees acquire the language skills necessary to find employment (Finn, 2010). Much like the United States, Australia has one of the highest refugee resettlement rates worldwide (Nicholls, 1998). Resettlement issues faced by Muslim refugee women during their first five years in Australia are similar to those encountered by refugee women in western countries. Casimiro, Hancock, and Northcote (2007) surveyed Australian women of refugee backgrounds during the first five years in Australia to affirm the importance of strong English language proficiency in securing meaningful employment and identified differences in religious beliefs and practices between the refugees and the host country as a significant barrier. Differences in religious beliefs and 
practices (Muslim refugees vs. Judeo-Christian Australian natives) created a significant barrier to the refugees' ability to secure meaningful employment (Casimiro, Hancock, \& Northcote, 2007). In addition to comparing the experiences of two groups of refugees (skilled versus unskilled Muslims), Fozdar (2012) also compared the data from 150 refugees of working age from three geographical regions (Europe, Middle East and Africa). Included in the sample were skilled Muslims versus non-Muslims who were proficient in the English language, and were educated and or had employment training (Fozdar, 2012). Results indicated that over 62\% of refugees in the study reported experiencing difficulty finding work in Australia due to discrimination, consistent with the findings of prior research by Casimiro et al. (2007). Those employed reported that accent, name, English language proficiency, appearance and religious practice were continuing sources of discrimination (Fozdar, 2012). Fozdar also examined the effect of religion on other aspects of integration and found Muslims in the sample felt no more excluded than other non-Muslim refugees. While these findings contradict the conclusions of other researchers who reported that skilled Muslim refugees have greater difficulty than other groups of refugees adapting to 'western-style' culture, Fozdar's conclusions demonstrate the complex nature of the challenge refugees face-even for those who are proficient in the English language, have received a formal education, and have prior professional work experiences from their native countries.

Employment and underemployment of refugees upon resettlement. The successful integration of refugees into society continues to be a growing concern for any western country that is on the receiving end of the refugee influx (Smith, 1996; ColicPeisker, 2009). Reports of high incidences of poverty, underemployment, and 
unemployment which lead to increased dependency on the government are common (Colic-Peisker \& Tilbury, 2006; Colic-Peisker, 2009). This has resulted in the creation of marginalized populations susceptible to exploitation, crime, and increased incidences of disease such as mental health issues and poor performance of refugee children in public schools, among other problems (Bloch, 2004). The 2015 Bureau of Labor Statistics report showed that foreign-born workers were more likely to be employed in the service sector versus their native-born counterparts. Service sector employment, which often leads to underemployment of refugees, includes jobs in production, transportation, construction, and maintenance occupations (Bureau of Labor Statistics, 2015). Underemployment typically results in refugees relying on government welfare benefits for many years (Casimiro et al., 2007). Possession of formal education and professional credentials and years of work experience from one's native country provides refugee women limited advantage in securing meaningful employment once resettled (Berger, 2004; Yashuko, 2006). Krahn et al. (2000) explored the issue of access to high-status occupations in the Canadian economy by surveying 525 refugees who previously held professional or managerial positions in their native country. The researchers discovered that compared to natives, the refugee population was slightly less educated, on average, with a higher proportion of the refugee participants lacking university credentials (Krahn et al., 2000). There were no significant differences in educational attainment among the sexes. The findings from the survey indicated that newly resettled refugees were more likely than other Canadians to be unemployed, and if employed they were more likely to have temporary or part-time jobs. Refugees who had previously held professional and or managerial positions in their native country reported difficulty in reentering their 
previous professions. At the time when this survey was conducted, only $25 \%$ of the refugees were employed in comparable positions (Krahn et al., 2000). Colic-Peisker and Tilbury (2006) also examined these issues of unemployment and underemployment in 150 refugees from Eastern Europe, Africa and the Middle East living in Australia. The researchers concluded that unemployment and or under-employment among refugees was brought about by factors that included an employer's lack of recognition of a refugee's formal academic and professional qualifications and work experiences from their native countries (Colic-Peisker \& Tilbury, 2006). As a result, refugee employment was concentrated in the secondary labor market that is typically comprised of low-status and poorly-compensated jobs that native-born Australian adults tended to avoid (ColicPeisker \& Tilbury, 2006). Tomlinson (2010) reported similar findings in the United Kingdom. When refugees arrive in the United Kingdom, they receive work authorization and assistance finding a job as quickly as possible. Regardless of pre-existing, postsecondary certifications, professional skills, and years of experiences working in the countries of their birth, many refugees start off working low skill, low pay jobs designed for unskilled workers (Tomlinson, 2010). Pietka-Nykaza (2015) also cited 'acceptance' and 'compromise' as coping strategies sometimes used by refugee physicians and teachers when working to re-enter their professions upon arrival in the United Kingdom. The other coping strategies identified were ambivalence and or withdrawal (PietkaNykaza, 2015).

These four studies confirm that refugees, upon arrival in their countries of resettlement, gravitate towards work in which they have previous experience (ColicPeisker \& Tilbury, 2006; Krahn et al., 2000; Pietka-Nykaza, 2015; Tomlinson, 2010). 
However, downward occupational mobility still persists, similar to the findings of early studies of Cuban and Vietnamese refugees in the United States (Finnan, 1981; Holloway, 1976; Portes, 1969). Employers acted as gatekeepers of white collar, professional and managerial jobs in all instances (Colic-Peisker \& Tilbury, 2006; Finnan, 1981; Holloway, 1976; Krahn et al., 2000; Pietka-Nykaza, 2015; Tomlinson, 2010). Regardless of a refugee's credentials and work experience, their status as refugees appears to preclude them from entry into the host country's workforce. However, what none of these studies accomplished was an examination of the cases from the employers' perspectives to understand what drives their actions toward exclusion (Colic-Peisker \& Tilbury, 2006; Finnan, 1981; Holloway, 1976; Krahn et al., 2000; Pietka-Nykaza, 2015; Tomlinson, 2010). It is important in a study examining a phenomenon central to refugees to gain, to some degree, the perspectives of all the actors involved for a better understanding of the outcomes. With such a focus on research on the experiences of refugees entering white collar professions, more studies are needed to explore the trend cited by the 2015 Bureau of Labor Statistics report that refugees are choosing to enter service sector employment.

\section{Strategies Used to Increase Refugee Employability}

The risk of unemployment is higher among refugees than their non-refugee counterparts (Renner \& Senft, 2013). In response to the growing numbers of refugees dependent on social welfare in Austria, Renner and Senft (2013) examined possible predictors of continued unemployment among refugees. They discovered refugees who completed training courses, had previous work experience in Austria, and had lived in Austria for two years had increased chances of employment versus those who had not completed training courses nor had previous work experience in Austria (Renner \& Senft, 
2013). These outcomes may be a consequence of the training courses taken but may also reflect a more motivated training participant from the outset. The researchers recommended that the vocational training of refugees should commence soon after arrival in Austria (Renner \& Senft, 2013). This theme was reflected in another empirical study that showcased how academic and or vocational training can serve as a means for obtaining stable, skilled entry-level employment with decent pay for refugees (Rupasingha, Ilvento, \& Freshwater, 2000). However, current training programs usually want participants to meet a set of minimum requirements, make extensive time commitments, and are usually not equipped to address limited English language proficient students (van Kooy, 2016). A proven alternative to the approach current programs have taken is the use of short-term vocational training designed to meet the refugees' need for appropriate education and work orientation while satisfying the demand of the local skilled-labor market (Rupasingha et al., 2000). The argument for refugees' participation in training programs is laid out in both studies, and while Rupasingha et al. (2000) addressed entry-level employment as the consequence of training, Renner and Senft (2013) highlighted the increased probability of finding work for disadvantaged refugees such as older, less educated refugees with poor language skills in Austria upon completion of training.

Levels, Dronkers, and Jencks (2014) investigated the observed skill disparities between adult immigrants and nonimmigrants. The researchers found of the 100,000 adults surveyed in 18 western countries, in almost all countries adult immigrants were less numerically skilled than non-immigrants, but the extent of the skills gap varied strongly across immigrants from different countries (Levels, Dronkers, \& Jencks, 2014). 
Differences were attributed to the immigrant population's demographics and socioeconomic characteristics as well as their previous employment history and countries of birth. The smallest skills gaps were evident in former socialist countries and the United Kingdom. In Cyprus and Canada, no skills gap between first generation migrants and natives existed, while in Ireland, first generation migrants were slightly more proficient than natives. In Cyprus, Ireland, Italy, and the United States, insignificant skills disparities were observed between natives and second-generation migrants, while in Canada, second generation immigrants outperformed native Canadians. The researchers concluded that skills gaps are smaller in more religiously diverse countries, in countries where immigrants are more strongly socially integrated, where labor markets are less protected, and where education is more strongly tailored to meet the education needs of immigrants (Levels et al., 2014). The findings are relevant to this study, as some trainees may have fled from countries where a women social status is tied to her role in the home, and therefore her access to educational opportunities may be lower than that of men, translating into women of refugee backgrounds that exhibit a greater skills gap and lower levels of labor market participation than that of women in the native population (Walter, 1981).

While it is important to know the job skills that refugees have, those skills they lack, and those they need when they first arrive in the United States, what is even more important is discovering the factors that enhance their ability to gain new and relevant skills for the U.S. labor markets. In 2013, Sulaiman-Hill and Thompson assessed the general self-efficacy-an individual's belief or confidence in their ability to exercise some measure of control over one's own motivation, behavior, and social environment in the 
face of stressful events to achieve a specific goal -of 186 Afghan and Kurdish refugees who were re-settled in New Zealand and Australia. The researchers found a positive relationship between general self-efficacy, mental health, and personal wellbeing (Sulaiman-Hill \& Thompson, 2013). The researchers thus recommended the use of successful role models to promote self-efficacy, increase motivation for learning, and help newly arrived refugees view resettlement as a positive challenge rather than an adversity. Sulaiman-Hill and Thompson's findings raise questions of whether graduate trainees serve as role models to current trainees or help motivate current trainees to complete their career transitions.

Historically, the United States chose to resettle blocks of refugees from a single region in the world into a given state or city (Refugees Community Plan, 2014). This resettlement allowed for service systems to secure interpreters, hire cultural liaisons, and make appropriate adjustments for the new population groups arriving in that locale. Resettlement practices such as these are no longer feasible because of the growing diversity in language and culture of refugees being resettled in the United States. Furthermore, in the 1970s, a refugee arriving in the United States had up to three years to achieve economic independence. Currently, refugees arriving in the United States are expected to attain the same economic independence within six months (Refugee Community Plan, 2014). If refugees are unable to accomplish this they are forced to enter the government welfare system which is ill equipped to meet their unique needs.

Community-based training programs. The need for economic independence has led refugees to increasingly rely on community-based programs where refugees, through their participation in the program, can acquire skills that lead to meaningful 
employment, and reduced reliance on government assistance (Finn, 2010; van Kooy, 2016). Community-based programs may be accredited or non-accredited. However, accredited programs are typically home/distance based learning offered by formal institutes of education (Buckingham, Marandet, Smith, Wainwright, \& Diosi, 2006). The focus of this research is non-credited programs, specifically sewing skills training programs offered by textile and apparel NPOs. Textile and apparel NPOs are organizations within the U.S. apparel industry which are classified under the nonprofit classification scheme (Cameron, 2004). Nonprofit organizations are neither business nor government organizations, but privately held organizations in various sectors governed by a voluntary board of directors (Cameron, 2004). These organizations exist for the good of the public and play a variety of roles in society that range from providing public services and advocating social concerns, to playing expressive roles in supporting creative and cultural activities. Still, some NPOs serve community building functions, while others act as nurturers of important national values (Cameron, 2004). As the visibility of NPOs grows in society, driven by their sheer number (11 million), the number of people they employ (7 percent of the U.S. workforce), and the 5.7 million people who serve as volunteers for these organizations, their role in society is becoming more important (Cameron, 2004).

Many community-based programs consider that the advantages of non-accredited training courses far outweigh those of accredited ones, primarily because they can draw non-traditional learners into adult education by providing a relaxed, safe and stress-free setting, where activities that are of interest to learners are taught (Buckingham et al., 2006). This is especially important as women trainees of refugee backgrounds may 
struggle with English language proficiency which can compound the stress trainees feel when starting a new training program, leading to negative training outcomes. Nonaccredited training programs also have the flexibility to be tailored to the specific needs of learners, therefore enabling progress at their own pace (Buckingham et al., 2006). In addition, they are usually available at a local and familiar venue as many training providers aim to run their programs in outreach centers (Buckingham et al., 2006). Prior textiles and apparel researchers have addressed issues of social responsibility such as fair trade (Goworek, 2011; Littrell \& Dickson, 1997) and environmental sustainability (Goworek, 2011) but neglected the role of textile and apparel NPOs in the resettlement and integration of refugees in the countries where they have been resettled.

\section{Theoretical Framework}

Throughout the literature, past researchers observed that adult learning motivation may well be a prerequisite for adult learning to take place (Knowles, 1990; Courtney, 1992). In other words, adult learning motivation may be the key to being successful in training programs. To encourage learning among mature adults, greater understanding is needed about the motivational factors that influence the decision to access or not access a learning opportunity. Theories from the adult education research have been utilized to explain women with refugee backgrounds' motivation to learn or take part in educational opportunities (Courtney, 1992). The motivational theories discussed in this literature review provided the theoretical lens to the questions on the impetus that drives women of refugee backgrounds to participate in career transitions purposefully setup by training providers in textile and apparel NPOs to achieve a set of outcomes. A general discussion 
of motivation and the two categories of theories that fall within its sphere, content and process theories are presented.

Motivation. When a person is motivated it means they are moved to do something (Ryan \& Deci, 2000). P. Kleinginna and Kleinginna (1981) described motivation as an internal state that serves to stimulate behavior and give it direction. This is critical to most aspects of life, especially in the area of employment and learning environments such as training. There are different orientations and levels of motivation (Ryan \& Deci, 2000) which are affected by numerous contextual factors (Cohen, 1990). In the textile and apparel industry, employee motivation is important to an organization's ability to effectively fulfil its mission.

In an organizational context, an organization's competitive edge is achieved through people (Pfeffer, 1994). Thus, the skills and performance of employees is important. Organizations attract talent by offering different types of benefits to employment that may come in the form of financial compensation, a healthy and safe working environment, achievement awards, work-life balance, professional development, mentorship, challenging work, medical benefits, educational programs, and retirement packages. Different types of benefits serve as sources of motivation in attracting, engaging, and retaining employees for a given organization. Winn (2013) indicated that employee behaviors and attitudes are affected by the extent to which they perceive that these different types of benefits offer a unique set of experiences and rewards. Although the most common type of benefit is gleaned from economic incentives, there are many intangible factors that motivate employees (Shore, Tashcian, \& Jourdan, 2006). Whether 
the benefit is tangible or intangible, it should increase the employee's sense of motivation (Shore et al., 2006).

Motivation for Training. Within the context of training, motivation to learn can be defined as a specific desire on the part of the trainee to learn the content of the training program (Noe \& Schmitt, 1986). Contextual factors such as supervisor's support and job attendance requirements can influence employee motivation. Cohen (1990) examined employees' motivation with regard to the pre-training environment at five organizations in the Midwest. The research focused on how supportive supervisors were, and whether employees felt training was voluntary or mandatory. Findings from the study showed support for Cohen's initial hypothesis that employees are motivated to participate in training if their supervisors are supportive and if they believe attendance at the training as voluntary versus mandatory (Cohen, 1990). When supervisors encouraged trainees to engage in training by asking questions, demonstrating understanding of the material, and encouraging application of learning to the workplace, all of which communicate supervisors' support, the likelihood of employee participation and degree of employee motivation increased. When supervisors were believed to be supportive, employees also believed that the skills and training they acquired during training would improve on-thejob performance, performance review which would lead to commensurate increases in salary and other benefits. Cohen theorized this was due to the fact that participants who lacked a college degree had less confidence in their own ability to learn and thus, feared the potential repercussions of poor performance in training programs. In these cases, the pre-training environment where perceptions of supervisory support and attendance norms are developed is a major factor influencing motivation. This study consisted of women 
of refugee background who may have lacked a college degree; thus, these two contextual factors may have impacted their pre-training and training experiences. In addition, the finding in Cohen's study that supervisors' support had a positive impact on the trainee's motivation to participate in the training program, demonstrated the importance of including training providers in this study as well, to understand their perspectives and experiences with women trainees of refugee backgrounds.

Motivation is an important factor in achieving training outcomes (Chen, Gully, \& Eden, 2004). Moreover, interview questions were designed that addressed participants' educational achievements, as well as past work experiences, to ascertain their psychomotor skill abilities that enhanced their ability to successfully complete the training program.

Theories of motivation. The motivational theory discussed in this literature review provided the conceptual lens to the questions on the impetus that drives participation or prohibits participation of women of refugee backgrounds in career training programs. The training programs were purposefully setup by training providers in textile and apparel NPOs to achieve a set of outcomes. Expectancy theory was the process theory of motivation used to identify the relationships among these factors (Vroom, 1964). This theory aligned with the purpose of this study which was to gain indepth understanding of the career transition experiences of women with refugee backgrounds within the context of textile and apparel NPOs in the United States by exploring what motivated them to participate in the training programs and attain training outcomes. 
Motives are the drivers of behavior and performance which contribute to humans' individualistic nature (Dinibutun, 2012). People have preferences among outcomes. For example, while one person may be driven by the need to feel secure, another may be driven by the need for public recognition. In organizations where productivity is largely dependent on employee performance, managers are interested in motivating their employees, especially since higher motivation has been linked with higher performance (Colquitt \& Simmering, 1998). Motivation is usually studied through an understanding of what people feel and how they think, which is also referred to as internal cognitive processes (Dinibutun, 2012). The cognitive theories of motivation are divided into content theories and process theories. Content and process theories seek to uncover underlying drivers of motivation. Content theory focuses on what motivates people in terms of identifying their needs while process theories address how people are motivated to meet their needs (Dinibutun, 2012). Content theories attempt to explain what constitute needs, drivers, or triggers of human behavior. These theories are concerned with identifying people's needs and their relative strengths, and the goals they pursue in order to satisfy the needs (e.g., Maslow's hierarchy of needs, Herzberg's two-factor theory). On the other hand, process theories attempt to identify the relationships (the how) among a set of dynamic variables, which make up motivation, and the actions required to influence behavior and actions. These theories focus on how an individual's conduct originates, is directed, and sustained. Process theories place emphasis on the actual process of motivation, and they provide a further contribution to the understanding of the complex nature of work motivation. Process theories focus on the psychological processes which affect motivation, with regard to the expectations, goals, and perceptions 
of equity (Dinibutun, 2012). Expectancy theory, a process theory, provided the theoretical perspective for this study. Therefore, process theories, and in particular expectancy theory will be discussed in the following sections.

Process theories. Applying expectancy theory (Vroom, 1964) to understanding how refugees were motivated to participate in career transitions assisted in understanding how training shaped their experiences. Victor Vroom, an expert in organizational behavior in the field of management, introduced the expectancy theory of motivation in his 1964 seminal work Work and Motivation (Victor H. Vroom, 2018). Expectancy theory is a cognitive process theory of motivation concerned with the cognitive antecedents to motivation and the way these antecedents relate to each other (Vroom, 1964). That is, expectancy theory is centered on the idea that people believe there are relationships between the effort they put forth, the performance they achieve from that effort, and the rewards they receive from their effort and performance (Colquitt et al., 2000). Simply put, people are motivated if they believe that applying more effort will lead to better performance and better performance will lead to desired rewards (Baumhof, Decker, Roder, \& Menrad, 2017). Victor Vroom (1964) was the first to develop a theory of motivation which applied to the workplace. This theory was later expanded and refined by Porter and Lawler (1968) and others, namely Pinder (1987). Expectancy theory (Vroom, 1964) is based on four assumptions. The first assumption is that people join organizations with expectations about their needs, motivations, and past experiences. These expectations influence how individuals react to the organization. A second assumption is that an individual's behavior is a result of conscious choice. That is, people are free to choose those behaviors suggested by their own expectancy calculations. A 
third assumption is that people want different things from the organization (e.g., good salary, job security, advancement, and meaningful work). A fourth assumption is that people will choose among alternatives so as to optimize personal outcomes (Vroom, 1964). Expectancy theory based on these assumptions has three key elements: expectancy, instrumentality, and valence (Vroom, 1964). A person is motivated to the degree that he or she believes that: (a) effort will lead to acceptable performance (expectancy), (b) performance will be rewarded (instrumentality), and (c) the value of the rewards is highly positive; this is also referred to as valence (Vroom, 1964).

Vroom (1964) suggested that motivation, expectancy, instrumentality, and valence are related to one another by the equation, and all three factors drive motivational force which drives behavior:

\section{Motivational force $=$ Expectancy $\mathbf{x}$ Instrumentality $\mathbf{x}$ Valence}

Each one of these factors has been given a value: expectancy ( 0 to 1$)$, instrumentality ( 0 to 1 ), and valence (-1 to 1 ). The multiplier effect in the equation means that greater motivational force will result when expectancy, instrumentality, and valence are all high versus when one or more of these factors are low (Vroom, 1964). The multiplier assumption of the theory also implies that if any one of the three factors is non-existent, the overall motivational force is zero (Vroom, 1964). If valence falls to less than 0 , then the motivational force will direct behaviors in the individual towards avoiding the reward or outcome (Lloyd \& Mertens, 2018).

Expectancy is a person's belief or anticipation that an increase in job-related effort will result in an increase in the given level of performance (Colquitt et al., 2000). According to Vroom (1964), a person's motivation will vary between a level 0 (no 
expectation) and a level 1 (full expectation) related to their belief that their efforts will accomplish a definite outcome. Instrumentality is an individual's confidence that an increase in a given performance will lead to various desired or anticipated rewards or outcomes (Colquitt et al., 2000). Vroom (1964) placed instrumentality on a range between a 0 (no expectation of desired outcome) and a 1(a reasonable probability exists of the conveyance of outcomes perceived). The third factor, valence, works on the premise that the reward or outcome is valued or preferred by the trainee over other rewards and outcomes, thus propelling the trainee to go after it (Colquitt et al., 2000). Vroom (1964) assigned this third factor in the motivational force equation, values between -1 to 1 . Thus, valence can be positive (whereby a trainee desires attaining the reward) or negative (whereby the trainee avoids attaining the reward). Therefore, salary increases, promotion, peer acceptance, recognition by supervisors, or any other intrinsic or extrinsic reward might have more or less value to individual employees or trainee. The value assigned to a training outcome is therefore dependent on its match to a trainee's depth of desire for that reward (training outcome).

Theoretically, a desired outcome has valence because it is related to an employee's needs, or in the case of this research study, a trainee's needs. Depending on other factors, a trainee's needs will dictate to some degree how desirable the training outcome is and may affect the level of motivation to attaining it.

Vroom's (1964) expectancy theory differs from the content theories of Maslow, Alderfer, Herzberg, and McClelland in that Vroom's expectancy theory does not provide specific suggestions on what motivates members of an organization or, for that matter, trainees in a training program. Instead, Vroom's theory provides a process of cognitive 
variables that reflects individual differences in work motivation. In this theory, employees or trainees do not act simply because of strong internal drives, unmet needs, or the application of rewards. Instead, they are rational people whose beliefs, perceptions, and probability estimates influence their behavior.

\section{Previous applications of expectancy theory}

Many empirical researchers have used Vroom's (1964) expectancy theory. In industry, Hayton and Cholakova (2012) used expectancy theory to understand employee motivation, while Candela, Gutierrez, \& Keating (2015) utilized the theory to understand career progression in the health sector. In the governmental sector, Park \& Kim's (2017) study of the federal employee workforce was conducted using the expectancy theory as the conceptual lens. In studies that were more closely related to the current research, Schepman and Richmond (2003) focused on links between expectancy theory and learned helplessness, and performance rating and pay. Helplessness occurs when people don't see a predictable relationship between their actions and the outcomes of those actions; they learn to believe that they have no control over those outcomes (Schepman \& Richmond, 2003). The researchers looked at one of the key conditions of expectancy theory, expectation. Employees should believe that they have some control over rewards or outcomes that are a direct result of their own actions. Schepman and Richmond study highlighted the fact that, employee helplessness may be a result of a perceived shortage of individual skills rather than feelings of low-expectancy.

Smith and Rupp (2004) looked at performance rating, pay scale, and motivation. Employees' motivation and morale improve when they are a part of the decision-making processes (Smith \& Rupp, 2004). This is borne out of a sense of ownership that comes 
about as the result of participating in the decision-making, ultimately leading to further engagement by the employee. Employees work harder and smarter when they are given more control over their work, and when they are encouraged to develop their skills and abilities (Pfeffer \& Veiga, 1999; Smith \& Rupp, 2004).

Champion (2008) conducted a study seeking the existence of a relationship between expectancy and improved performance. The researcher predicted that the use of expectancy theory, using expectancy, instrumentality, and valence as part of a pretraining module would have a positive effect of increasing employees' engagement in their work and its outcome on the division in general (Champion, 2008). However, the results were inconclusive. The researcher determined that the application of the theory could be used in other training initiatives to create a positive impact on the training results (Champion, 2008).

This theory suggested that trainees, like employees, have preferences among the different outcomes or rewards that can result from participation in training. Trainees also have expectations regarding the likelihood that effort invested in training will result in mastery of training content (i.e., expectancy). Expectancy theory can be useful for predicting behavior when it is under the employees' control, the work environment provides conditional rewards, and behavior-outcome relationships are clear (Mitchell, 1982). Because these conditions are usually met in a training context (e.g., attending training is under the employee's control and is purported to result in positive outcomes), this theory has frequently been used to understand training motivation (e.g., Mathieu \& Martineau, 1997). 
Mathieu and Martineau's (1997) model of training motivation suggested that individual and situational characteristics impact training outcomes by affecting expectancy theory variables and training motivation. In terms of individual characteristics, Mathieu et al. (1992) suggested that achievement motivation was related to training outcomes through the mechanism of self-efficacy. Colquitt and Simmering (1998) presented a model in which conscientiousness related to training outcomes by affecting expectancy and valence. In terms of situational characteristics, Quinones (1995) posited that aspects of the training assignment related to training outcomes through the mechanism of self-efficacy.

This research used a qualitative approach because very little was known about the topic, and an exploratory approach was appropriate to uncover the factors that facilitated or hindered motivation in trainees of refugee backgrounds to participate in training and attain training outcomes (Yin, 2012).

\section{Research Gap}

Some refugees arrive in the United States with few skills, credentials that are not recognized, and few established social networks (Holloway, 1976; Kogan, 2003; Berger, 2004). Nonetheless, it is imperative that they find employment soon after arrival, regardless of whether their skills and education match up to the needs of the U.S. labor market. There is a lack of literature on the topic of the training programs in U.S.-based textile and apparel NPOs, how these organizations provide training opportunities that build skills or lead to the skills acquisition by refugees, and what motivates trainees with refugee backgrounds to participate in these career transition programs and attain training outcomes. 
The purpose of this study was to gain in-depth understanding of the career transition experiences of women with refugee backgrounds within the context of textile and apparel NPOs in the United States by exploring what motivated them to participate in the training programs and attain training outcomes. A multiple case study approach was used to study graduate and current trainees of formal skills training programs; training providers from each textile and apparel NPO were interviewed on their organization's structure, vision and mission. The research sites were limited to textile and apparel NPOs located in a large urban area in a state located in the northwest region of the U.S.

To accomplish this purpose, the research questions are:

Research Question 1: What facilitates the motivation of women trainees of refugee backgrounds to participate in career transition programs and attain training outcomes?

Research Question 2: What hinders the motivation of women trainees of refugee backgrounds to participate in career transition programs and attain training outcomes?

Interviews and direct and participant observations were the primary methods of data collection. This qualitative research study helps fill the gap in the literature on what motivates women of refugee backgrounds to participate in career transition programs offered by U.S.-based textile and apparel NPOs.

\section{Summary}

Based on the review of literature presented, expectancy theory provided the theoretical perspective to the questions on the impetus that drives participation or prohibits participation of women of refugee backgrounds in career training programs. This study proposed a multiple case study approach to explore the career training 
experiences of women trainees with refugee backgrounds. Training providers in the textile and apparel NPOs were also participants in this study. 


\section{CHAPTER III: METHODOLOGY}

Chapter III contains the following sections (a) researcher's philosophy, (b) research methodology and design appropriateness, (c) Elaboration and rational for research design, (d) the role of the researcher, (e) research questions, (f) sample size, (g) geographic location, (h) instrumentation, (i) data collection timeframe, (j) data analysis, and (k) summary.

\section{Researcher's Philosophy}

A researcher's epistemological perspective underlies and permeates every phase of the research process (Merriam, 1998). Some qualitative research studies are founded on the constructivist philosophical assumption that individuals interacting in the society construct the reality within which they live in (Merriam, 1998). This means that researchers that subscribe to this assumption are interested in understanding how people make sense of their lived experiences in this world (Yanzan, 2015). Each researcher brings in their own construction of reality to the research setting which interacts with the research participants' construction of the phenomenon being studied (Merriam, 1998). The final product of this type of study is yet another interpretation by the researcher of others' views filtered through their own experiences of the world (Merriam, 1998).

As an emerging researcher in the clothing and textiles discipline, epistemologically I position myself closely to the constructivist ideology. I believe knowledge is created in the minds of people through social interaction and may manifest through individuals' social practices. In essence, social reality is constructed by individuals and exists within the realm of the mind.

The epistemological lens of this qualitative multiple case study is based on the philosophical premise of constructivism and interpretivism. Researchers that subscribe to 
the ontological philosophy that truth is relative and dependent on an individual's outlook are constructivists. Constructivism is centered on the idea that reality is socially constructed (Searle, 1995); meaning that human beings create meaning. Crabtree and Miller (1999), stated that a key advantage of this approach is the close cooperation between the participant and the researcher, while enabling research participants to tell their stories. It is through this method that research participants' views or realities are brought into focus, enabling the researcher to better understand participants' actions (Lather, 1992; Robottom \& Hart, 1993).

The qualitative characteristic of the study approached the phenomenon of career transition experiences of women of refugee backgrounds through the interpretation of their real-life experiences in training programs offered by textile and apparel NPOs. The use of the interpretative lens allowed for a deeper understanding of the research into the phenomenon and for the discovery of issues that existed within it (Leedy \& Ormond, 2010). As the researcher spent time in the field, became the data collection instrument, and collaborated in the natural setting of the research participants, the researcher gained insights and firsthand information of the phenomenon. Through the constructivist perspective, current and graduate female trainees with refugee backgrounds and their training providers detailed their experiences through semi-structured interviews (Creswell, 2007). The natural world (career transition program) of the research participants provided the setting for all participant observations and shared experiences.

Ontologically, I believe multiple realties exist that are shaped within the context of the textile and apparel NPO's training program. These truths may evolve and change with each participant's time and experience within the training program. This was evident 
through the elements of this qualitative inquiry (Stake, 1995), where the reality of the perception of a participant's reality was through their own interpretation. This is because

reality is socially constructed and people create meanings of their experiences. Therefore, this qualitative multiple case study described the career training program that two groups of research participants had experienced -current and graduate trainees. Training providers were also included in the study to provide context-related information on the career transition programs as well as to confirm the information garnered from interviews with trainees, thereby minimizing investigator bias. Using semi-structured interviews, the research participants revealed their subjective career transition experiences. The semistructured interviews with trainees and training providers, allowed for knowledge exchange of what was known and what the researcher sought to understand as reality. Therefore, the qualitative multiple case study was selected as the design for this research study. However, the researcher adopted a flexible research design approach for this study to allow for the natural research design for the study to emerge during data collection.

\section{Research Methodology and Design Appropriateness}

Elaboration and rational for research methodology. The purpose of this study was to gain in-depth understanding of the career transition experiences of women with refugee backgrounds within the context of textile and apparel NPOs in the United States by exploring what motivated them to participate in the training programs and attain training outcomes. In this study, a career transition occurs through participation in a formal training program (Liptak, 2008).

A case study approach was used because it usually involves the comprehensive exploration of complex and dynamic physical and social phenomena, using a variety of 
sources of information, yet retains a holistic and real-world perspective. (Yin, 2015;

Baxter \& Jack, 2008). A case may include a person, organization, phenomena, event, media, language, community, artifact, and / or cultural product (Baxter \& Jack, 2008). The case study methodology sets itself apart from other approaches in two ways. First, it is centered on the comprehensive study of complex social units. Second, it maintains the cohesiveness of the social unit throughout the research process (Roller \& Lavrakas, 2015). Yin (2014) states that a case study approach is appropriate when: (a) the study seeks to answer "why" and "how" questions; (b) participant behavior cannot be manipulated; (c) context is important to the phenomenon being studied; (d) there is no clear boundary between the phenomenon and the context; (e) there are more variables of interest than data points - the data point being the "case" being explored; and (f) there are multiple sources of evidence. So the difference between the case study approach and other common qualitative approaches is the contemporary context, positioned within boundaries, and the multiple perspectives that underlie case study research as a method that supports the inquiry of complexity (Taylor, 2013).

The methods used to direct this qualitative multiple case study were typical of case study methodology (Stake, 2006). The study utilized an embedded multiple case study design (See Figure 1). According to Yin (2012), multiple case design may involve two cases or more that intentionally try to assess the conditions under which the similar findings might be replicated. On the other hand, the multiple cases might include cases that are intentionally contrasting. Multiple case study design allowed the investigator to explore career transitions though the use of a replication strategy, carried out in two separate stages: (1) literal replication stage and, (2) theoretical replication stage (Yin, 
1994). In the literal replication stage, current trainees were chosen to obtain similar results, while in the theoretical replication stage, graduate trainees were chosen to explore and confirm or refute the patterns identified in the primary cases. According to Eisenhardt (1989), in this type of case study, if all or most of the cases provide similar results, then there can be substantial support for the development of a preliminary theory that describes career transitions. On the basis of analytic claims, the preliminary theory on career transitions may be used to inform what facilitates or hinders motivation in learning situations of adults with low educational attainment or with backgrounds of trauma. However, the development of a preliminary theory was beyond the scope of this study. Instead a conceptual model explaining the conditional factors that facilitated training behavior and outcomes among the trainees was developed.

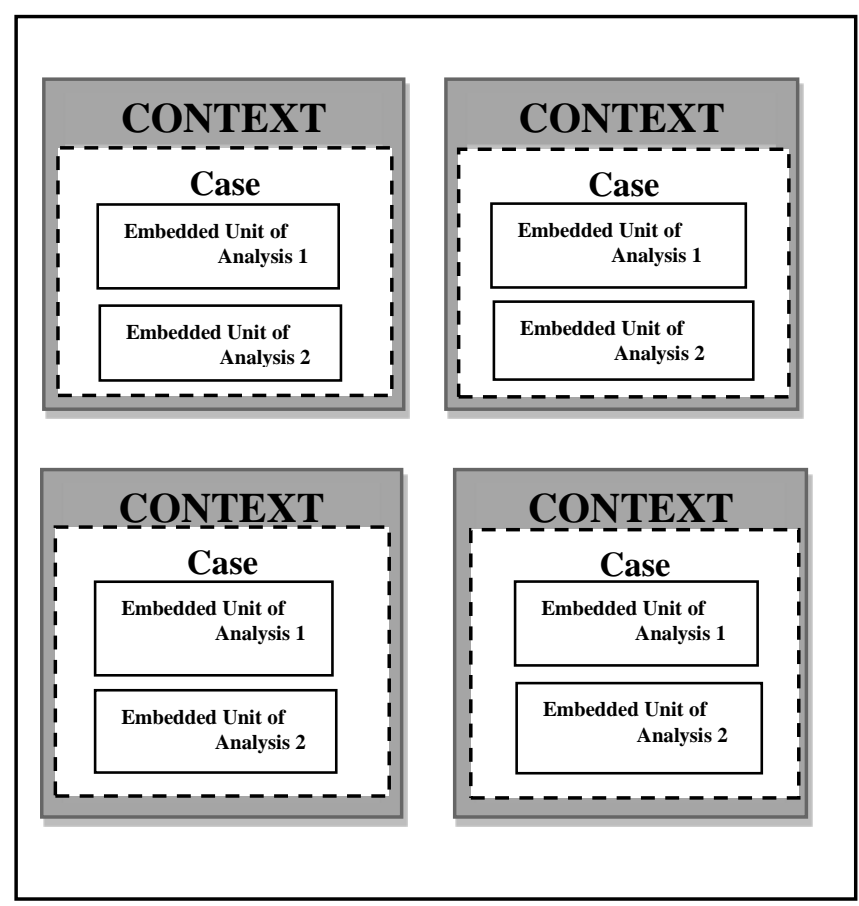

Adapted from: COSMOS Corporation

Figure 1. Embedded multiple case design (COSMOS Corp., 1989) 
This type of methodology assumed a broader study of the skills training programs in textile and apparel NPOs within which the career transition phenomenon is embedded (See Figure 2). This broader method addressed the overall nature of career transition programs in five textile and apparel NPOs, and explored how different factors influenced motivation to participate in career transitions and, ultimately, the attainment of training outcomes. Within this context, multiple case studies provided a particularly helpful way to address key career transition issues by capitalizing on the holistic approach to studying a phenomenon and the use of replication logic (Yin, 2003) to generate a credible conceptual model.

Case studies allow for a range of information that applies to a phenomenon to be taken into consideration all at once. Since a case is by definition, a single unit, findings cannot be generalized to statistically represent other entities. This, however, should not be treated as a shortcoming, because the logic of embedded case studies and the epistemological underpinning upon which they are grounded differ from those of statistical methods (Yin, 2003). The logic of embedded case study analysis follows Yin's (2003) rationale that inferences from multiple case studies reflect a "replication logic." Replication logic generalizes to theory rather than to a population. That is, analysis of multiple cases allows for the generation of a theory, or in this case a conceptual model, consistent with the case data. Such theories or conceptual models, based on the embedded case study analysis, cannot be automatically generalized to a population based on this approach. 


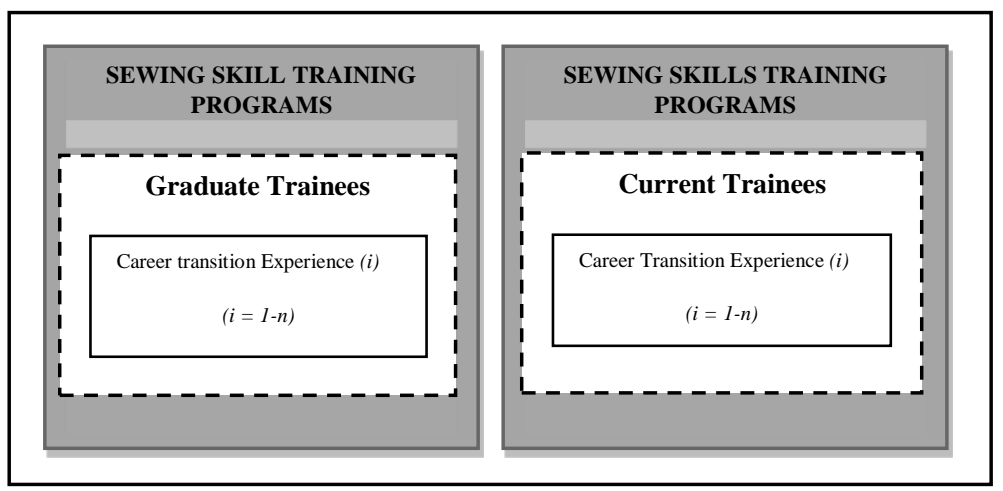

Adapted from: COSMOS Corporation

Figure 2. The application of embedded multiple case design

(COSMOS Corp., 1989).

\section{Elaboration and rational for research design}

The embedded multiple case study method required several phases. The first phase involved selecting the criteria for which the textile and apparel NPOs would be chosen. This was finalized before data collection ensued. The textile and apparel NPOs were all located in a large city in the western region of the U.S. The location was selected based on the current re-vitalization of the sewn products industry in the area by local businesses (Rayna, personal communication, November 4, 2016). The researcher's experience with an apparel industry expert involved with training programs in the area and the availability of participants with the personal career transition experiences to answer the interview questions were also important determining factors. There were four criteria used to select the textile and apparel NPOs, each organization needed to:

(a) Be a 501(c)(3) NPO

(b) Offer a sewing skills training program targeted to women with refugee backgrounds within the last three months. Several textile and apparel NPOs were agreed to be a part of the study, but due to a lack of funding they had not offered training for over three months from the time data collection occurred. 
(c) Have training providers with firsthand knowledge of their training programs and who would be willing to participate in the study and help with recruitment of program trainees and graduates

(d) Utilize Avenio's project-based sewing curriculum, whereby each additional project increased complexity and skill advancement. Avenio's sewing curriculum was shared among training providers and utilized to varying degrees within the individual training programs.

When identifying and recruiting research participants, the researcher did so with the research purpose and research question in mind (Leavy, 2017). The sampling focused on the career transition experiences of women with refugee backgrounds in multiple textile and apparel NPOs in the United States, and, their training providers were also interviewed. Purposive sampling techniques were utilized to select potential participants for in-depth study, so that the best data was collected. According to Patton (2015), researchers can get insight that addresses the research purpose and questions by interviewing appropriate participants. These participants consisted of individuals that the researcher gathered information on their career transition experiences, which was the phenomenon central to this research study. For purposive sampling to be possible, the researcher found several training providers who served as interviewees, as well as key informants in the field by helping to identify potential participants.

A combination of two sampling strategies was used for this study, maximum variation (heterogeneity) sampling and snowball sampling. Maximum variation sampling occurs when a researcher chooses a wide variety of participants to make up their research sample in order to understand how different groups of people view a specific 
phenomenon (Roy, Zvonkovic, Goldberg, Sharp, \& LaRossa, 2015). In this research study, a heterogeneous sample was deemed appropriate, because, by speaking with the training providers and women with refugee backgrounds who were still in training or had completed their training, multiple perspectives on career transition experiences would emerge confirming or disconfirming the evidence needed to answer the over-arching research questions of the study.

The second sampling strategy used was snowball sampling. Snowball sampling is the process whereby the researcher seeks information from key informants or other research participants about other potential participants that leads to the recruitment of other research participants (Patton, 2015). In this study, select research participants who were identified by training providers were directly asked at the completion of their interviews to recommend other participants. Direct requests for recommendations of potential participants were also made to training providers by the researcher during participant observations.

To understand the career transition phenomenon, different types of participants (i.e. trainees and training providers), from different textile and apparel NPO training programs, and who participated in the training programs at different times (i.e. graduate trainees and current trainees) were selected. Homogenous sampling was used to select appropriate sub-units (participants) for each case represented. Participants selected using homogenous sampling aligned with the researcher's aim to gain in-depth understanding into each case's career transition experience because the selected participants were likely to have comparable results (Patton, 1990; Yin, 2012). The researcher checked each 
potential participant against the inclusion criteria generated in relation to the purpose of the study.

Data quality can be improved by increasing richness and depth when the researcher makes appropriate decisions about the units of observation and the units of analysis of the study (Roy, Zvonkovic, Goldberg, Sharp \& LaRossa, 2015). The unit of observation, which is also known as the unit of measure, is defined as the "who" or "what" for which data are collected (Sedgewick, 2014). The unit of analysis (case) is defined as the "who" or "what" for which data are analyzed and conclusions drawn (Sedgewick, 2014). In this research study that explored the career transition experiences of female trainees with refugee backgrounds in multiple textile and apparel NPOs and their training providers, the unit of observation was the individual graduate and current trainees and their training providers. The unit of analysis was the individual career transition experience narrated by each trainee.

Yin (2003) and Stake (1995) recommend that a case study have boundaries to avoid it becoming outsized. According to Creswell (2003), a case can be bound by time and place, Stake (2008) suggests that the same can be done through time and activity, while Miles and Huberman (1994) posit that the same can be achieved by definition and context. In this exploratory study involving women trainees of refugee backgrounds and their training providers, describing their career transition experiences, established boundaries would need to include a definition of what a career transition program was and which women trainees of refugee backgrounds and training providers would be sampled. This helped retain the focus in the study and avoided the study becoming unwieldy. The researcher also specified the types of organizations these women had to be 
receiving the career transition training from. Thus, the binding of the case was achieved by definition and context (Miles \& Huberman, 1994). It was important to bind the case to establish what was studied and what was not, which was also indication of the breadth and depth to which the study went (Baxter \& Jack, 2008).

This research design enabled the researcher to interpret and understand the experiences of the research participants through the interpretive lens. In essence, through the lens of qualitative design, the interpretation and understanding, what was revealed were answers to both of the study's research questions; what facilitates or hinders the motivation of female trainees of refugee backgrounds to participate and/or attain training outcomes.

\section{The role of the researcher}

In qualitative research, the researcher is considered the instrument, "meaning that the qualitative researcher wields substantial control in the design content, the outcomes, and the interpretations of the research." (Roller \& Lavrakas, 2015, p. 207). In this study, the researcher was the data collection instrument when data was collected through interviews, documents and artifacts, and observations. The researcher's responsibilities included: (a) the recruitment of participants, (b) the creation of interview and observational protocols, (c) the interviewing and analysis of the data collected through interviews, (d) the recording of observations in the field, (e) the answering of the study's research question from data analysis results, and (f) the finalization of the research study's conclusion and recommendations. The research study was conducted on female trainees of refugee background and training providers in textile and apparel NPOs. 
The researcher's affiliation with the topic, participants, and research area was from past personal friendships with refugees in the community and church she attended, and a previous research study she conducted on a small sewing skills program offered to recently resettled refugees by a faith-based organization in her community. Having several friends with a refugee background, the researcher observed how they struggled with being preliterate, not possessing English language proficiency, having few marketable jobs skills, and yet needing to support large families, created barriers to them accessing vocational training opportunities. Many of these friends often expressed frustration, but also had the optimism to keep forging ahead. The desire to bring to the forefront the experiences of these women prompted me to investigate the problems more in-depth.

For this research study, the researcher was only acquainted with one of the study participants - Rayna, who served as a gatekeeper to several of the textile and apparel NPOs that the researcher conducted her study on. The only sewing skills training program that the researcher was familiar with was the one offered by a faith-based NPO in her community, whose participants had been the subjects of an earlier study. Therefore, the researcher's understanding of the topic came from her personal friendships, the literature review, and her previous study on sewing skills programs in the United States.

Protocol. Prior to collecting data, the researcher conducted a self-reflection to document biases and expectations regarding the potential findings of the study. Biases and expectations related to: (a) real and or perceived difficulty in accessing the participants needed for the study, (b) the implicit and or explicit expectation of 
participation by training providers and their influence on how learnings are communicated, (c) the English language proficiency of refugee trainees and providers and their ability to understand the questions, and (d) the ability to quickly establish rapport with the participants because the researcher did not have any prior contact with the participants before arriving at the data collection sites. The researcher also worked to reduce bias when collecting data and during analysis, to ensure the integrity of the information gathered. Data gathered from the interviews were utilized in support of themes and conclusions produced in the study. The researcher worked to maintain a friendly, but neutral and objective demeanor with participants during the interviews. Stuckey (2013) stated that a researcher's biases can be reduced if his or her personal views are not expressed in response to a participant's comments during the interview.

The researcher kept a reflexive journal chronicling the impact of her presence on the environment being studied and also on the participants being studied and observed. A potential negative consequence of the researcher being in the natural environment in which the central phenomenon was occurring was the potential of changing the dynamics of the environment, and, thereby, negatively affecting the quality of the research design (Roller \& Lavrakas, 2015). Furthermore, the researcher engaged with the training providers after the conclusion of data collection activities and asked them to describe any observable changes to their training environment that had occurred.

To establish rapport with current trainees affiliated with each organization, the training providers gave the researcher the opportunity to attend ongoing training sessions before commencing with the semi-structured interviews. The intent was to allow training providers and current trainees to become acquainted with the researcher first. The 
researcher also shared a brief presentation about African textiles as a way of connecting on a personal level with training participants and used this platform to segue into a short overview of the research study. To access women graduate trainees of refugee backgrounds who had graduated from the training programs, the researcher relied on training providers to not only recommend other program graduates, but also formally introduce the study and the researcher to these program graduates before she established first contact.

Triangulation. Descriptive coding of these qualitative data, assisted in the documentation of the types of tangible goods that the graduate and current trainees in the training programs made, manipulated, and experienced during their career transition processes. The use of multiple sources of data that is characteristic of case study research made descriptive coding ideal for the research design utilized in this study, because the coding method could be applied to interview transcripts, field notes, journals, documents, correspondence, physical artifacts, and video (Saldaña, 2014). Yin (2013) stated that methodological triangulation, which is the use of multiple sources of evidence, helps reduce bias, and in doing so may increase credibility and improve the trustworthiness of the study. The interview transcripts were analyzed, while documents such as observational protocols and field notes helped provide context and corroborate interviews.

The open-ended nature of the semi-structured interview protocol allowed the researcher to clarify participants' responses through further discussion, thereby reducing the researcher bias even more (Cowan, 2014). The use of member checking to also clarify and correct statements that participants made during the course of their interviews, to 
avoid distorted meanings helped reduce researcher bias in the study and was done as the interview unfolded.

The researcher used multiple sources of data to explore the participants' experiences in career transition programs, but the interviews were used as the primary source of data while the other sources of data provided context and corroborated interviews. All research participants were interviewed using a semi-structured interview protocol, which was developed after a review of the literature. The methodology and methods used in this study promoted the in-depth exploration of the phenomenon by using different perspectives to reveal the many facets of career transition experiences so it could be better understood.

\section{Research Questions}

The research questions developed for this study (see Table 1) were used to assist in scaling the scope of the study further (Yin, 2014). 
Table 1.

Research Questions and Corresponding Interview Question examples

\begin{tabular}{|c|c|c|}
\hline $\begin{array}{l}\text { Research Question } \\
\text { (RQ) }\end{array}$ & $\begin{array}{l}\text { Examples of Interview } \\
\text { Questions to address } \\
\text { research question (current } \\
\text { trainees) }\end{array}$ & $\begin{array}{l}\text { Examples of Interview } \\
\text { Questions to Address } \\
\text { research question } \\
\text { (graduate trainees) }\end{array}$ \\
\hline $\begin{array}{l}\text { RQ1: What facilitates } \\
\text { the motivation of } \\
\text { women trainees of } \\
\text { refugee backgrounds } \\
\text { to participate in career } \\
\text { transition programs } \\
\text { and attain training } \\
\text { outcomes? }\end{array}$ & $\begin{array}{l}\text { What are some of things you } \\
\text { believe completing this } \\
\text { training program will allow } \\
\text { you to do for you yourself } \\
\text { and/or your family? }\end{array}$ & $\begin{array}{l}\text { What made you believe } \\
\text { that you had the ability to } \\
\text { learn the skills and would } \\
\text { complete the program? } \\
\text { What kept you } \\
\text { participating in the } \\
\text { program until completion? }\end{array}$ \\
\hline $\begin{array}{l}\text { RQ2: What hinders } \\
\text { the motivation of } \\
\text { women trainees of } \\
\text { refugee backgrounds } \\
\text { to participate in career } \\
\text { transition programs } \\
\text { and attain training } \\
\text { outcomes? }\end{array}$ & $\begin{array}{l}\text { What are some of the } \\
\text { challenges you face while } \\
\text { participating in the training } \\
\text { program? }\end{array}$ & $\begin{array}{l}\text { What are some of the } \\
\text { challenges you faced and } \\
\text { had to overcome to } \\
\text { complete the training } \\
\text { program? }\end{array}$ \\
\hline
\end{tabular}

\section{Sample Size}

Guidelines persist in the literature about appropriate sample sizes for qualitative studies. When it comes to multiple case study design, qualitative methodologist Creswell (2002) recommended three to five cases as an appropriate sample size, while Yin (1994) advised that six to ten sub-units per case in an embedded multiple-case study were adequate. However, given the variance in the quantity and quality of data collected in qualitative multiple case studies, these guidelines may be misleading and impede the purpose of the research study (LaRossa, 2012). For example, Knappert, Kornau and 
Figengul (2017) used a qualitative inductive approach to study what shapes the inclusionexclusion experiences of refugees at work of twenty male and female refugees who were part of a critical case study, while, Abkhezr, McMahon, Glasheen and Campbell (2017) used the narratives of three refugee women living in Australia to understand the career development processes they had undergone and in order to suggest new strategies of combatting the challenges they faced in finding appropriate work. Both studies used the multiple case study approach with special attention paid to understanding the lived experiences of refugees in the context of work. However, we cannot fully appreciate the contribution each one of these studies makes based just on their given sample size because the main goal of all qualitative research is to provide insight on a specific phenomenon; making generalizations from qualitative multiple case studies are secondary goals. Ultimately, the sample size was determined by the number of units/subunits needed to reach saturation. The final sample size for this research study was: seven current trainees, seven graduate trainees and ten training providers.

\section{Geographic Location}

The state of Washington, located in the U.S. Northwest is ranked seventh among

U.S. states in refugee resettlement, with its capital city being the most popular destination (U.S. Department of State, 2017). The U.S. Department of State (2017) estimates that of the total number of refugees resettled nationwide since 2003, the aforementioned U.S. state accounts for 4.3 percent, or 32,898 of them. The majority of the refugees being resettled in the state come from Muslim-majority countries, such as Somalia and Iraq (U.S. Department of State, 2017). It is the responsibility of the five voluntary refugee resettlement agencies (VOLAGS) in the state of Washington to assist refugees in finding 
affordable housing, enrolling their children in school, applying for benefits, and looking for job training and work opportunities (U.S. Department of State, 2017).

Access to Sample. One of the challenges of case study methodology research is gaining access to research participants, especially from a traditionally hard-to-access population like refugees. Of the many recruitment methods researchers have proposed, the one deemed most effective is through personal contacts. In 2014, three years before this research was conducted, the researcher met Rayna -a board member at a textile and apparel NPO, located in the same large city in the state of Washington, and began the process of establishing rapport. Over the course of two years rapport was established around a common interest to help women with refugee backgrounds successfully integrate into their new communities. By the time the concept of this study was introduced, Rayna urged the researcher to consider the textile and apparel NPO (Avenio) where she served as board member as one of the data collection sites used in the study. With the help of Rayna, sewing skills training programs offered by several other textile and apparel NPOs based in that city were evaluated and the ones that met the four parameters outlined by the researcher were selected and introduced to the research study with the hope that their interest in the study would translate into a willingness to participate and actively recruit potential research participants (see Table 5).

The next phase of this study involved inviting five of the selected textile and apparel NPOs to participate in the research study (See Table 5) and through which all research participants for the study were recruited. The data collection sites were purposefully selected because they provided the researcher access to key research participants who were either graduate or current trainees of the career training programs, 
the artifacts and documents that would illustrate the processes and tools used for career training by trainees, and provide answers to the research questions (Miles \& Huberman, 1994). The selected participants needed to be hospitable to the research study so that they could provide answers to the research questions. In this study, data collection sites were pre-approved for the researcher to conduct her study by key-stakeholders in each organization. This allowed the researcher to gain access to participants from each organization with the help of each organization's leadership and generate interest in participation in the research study among graduate and current trainees and their trainers.

\section{Instrumentation}

This multiple case study used past and present training program contexts to understand the similarities and differences of career transition experiences of graduate and current female trainees with refugee backgrounds. To ensure construct validity, the researcher began by using relevant literature to operationalize the key concepts in the study into a set of measures, to minimize researcher bias. The questions on the interview protocol for graduate and current female trainees with refugee backgrounds were developed via a literature review on the topics of career counseling, motivation, expectation, refugee integration and personal agency to answer the research question and research sub-questions in this study. The current research study was exploratory in nature and sought to understand a participant's experience while making a career transition. A semi-structured question format was required in order to elicit rich data or thick description from participants that would reveal how they created reality and regarded their career transition experiences (Yin, 2012). Questions were also developed using the literature on motivation and refugee integration to address intrinsic and extrinsic 
motivators, and personal re offered to trainees of refugee background by the textile and apparel NPOs. These questions were semi-structured in nature to elicit rich data. Both interview protocols began with a series of screening and demographic questions before initiating the questions that addressed participants' personal experiences in career transitions program. All semi-structured interviews were audio-recorded and transcribed by the researcher following the interview.

\section{Informed Consent}

The researcher was responsible for ensuring that the study met the three principles for academic research (beneficence, the respect of an individual's rights and dignity, and justice) stated in the Belmont Report (U. S. Department of Health \& Human Services, 1979). This was done through attaining informed consent from each participant once each individual understood the purpose of the study and what participation in the study entailed. All participants in the study were at least 18 years old, women with refugee backgrounds participating or had participated in a career transition program, or training providers for these women, and English-speaking.

The researcher proceeded with the recruitment of training providers only after obtaining the University of Missouri's IRB approval. Then, the researcher proceeded to send an email to Rayna (board member in one of the five organizations, who had helped introduce the researcher's study to the leaders of the textile and apparel NPOs and gain approval) attaching a formal letter of introduction to the researcher and the study. The letter served as a formal invitation and request for permission to collect data on-site. The letter stated the purpose of the study as well as method for data collection, how confidentiality would be addressed, and the storage of collected data. Once the researcher 
received an approval via email, this signaled that entry to the data collection site to conduct direct observations, semi-structured interviews, as well as examine physical artifacts was granted to the researcher. This formal approval also signaled that training providers would assist the researcher with the recruitment of graduate and current female trainees with refugee backgrounds. Every participant that was interviewed as a part of this study was given the validated IRB approval form, and the option not to participate. Those choosing to proceed completed and signed the informed consent documents in accordance with the University of Missouri's IRB requirements for adult participants in research studies. There were no incentives available in return for their participation in the research study. The researcher provided participants with her contact information so that they could contact her should any questions come up once she had left the location or completed the interview process.

Due to the nature of this research study, during data collection and analysis, the researcher protected the anonymity of the NPOs and participants by disassociating names from responses throughout the study. Pseudonyms were used in place of actual participant names and NPOs' names to maintain confidentiality. Data records will be retained by the researcher for five years and then disposed in an approved manner.

\section{Pilot Study}

A pilot study was conducted to validate the questions in the interview protocol by people of similar backgrounds as the trainees of refugee background. The researcher could not validate the interview protocol for training providers on actual training providers in sewing programs so a graduate student in the department of rural sociology was used as a substitute. Two women of refugee background who had a fair command of 
the English language and had once participated in a local sewing program participated in the pilot study. The results of the pilot study were twofold. First, there was a refinement of the questions on both interview protocols in the clarity and reduction in the questions asked. So, for example, several of the questions on the trainees' original interview protocol asked about educational background and training background were combined into one question that served to encompass both; "Tell me about your schooling." Other changes on both protocols addressed the order in which interview questions were asked, and, so, re-ordering occurred to help with a more natural progression of the discussion during the interviews. Secondly, the data from the pilot study helped the researcher ascertain whether the data collected answered the research questions. This led to deletion of some questions on the interview protocols, as they were deemed irrelevant to the research questions. A few questions were added based on feedback received from participants.

\section{Protocol, Recordings and Coding}

For women trainees of refugee backgrounds, the interview protocols were developed with questions focused on the: (a) individual's general background and resettlement experiences, (b) schooling and career history, and (c) expectations and experiences while in the career transition program offered by the textile and apparel NPO. For training providers, the interview protocol concentrated on their: (a) general background, (b) fashion-related education and experiences, (c) experiences working with trainees of refugee backgrounds, (d) current roles in the training program or textile and apparel NPO, and (e) observations about factors that promote successful and unsuccessful career transitions of trainees of refugee backgrounds. An interview protocol was used in 
each participant interview in this study. However, the researcher allowed for flexibility and adjusted and added interview questions as the interviewing progressed. For example, early on in the interviewing process when the researcher learned through interviews with several training providers that multiple instructors were employed in instructing trainees, a question on the trainee's interview protocol that addressed the use of multiple instructors in the training program was added.

Twenty-four of the twenty-seven interviews were recorded on a digital recorder once participants provided Informed Consent to participate in the study and manually transcribed by the researcher. Three participants were not included in the study because they were either the wrong gender or had never held refugee status in the United States. All graduate and current trainees in this research study were women with refugee backgrounds. One interview was conducted in Swahili because of the trainee's lack of English language proficiency. The interview was later translated into English and then transcribed by the researcher before being checked for accuracy against the original interview.

Throughout the transcription and analysis of the interviews, the researcher was engaged in analytic memoing. Miles, Huberman and Saldaña (2014) describe analytic memoing as the preliminary attempts by the researcher to transform data into "higher level analytical meanings" (p. 95). This was done by the researcher writing down or tape recording her initial impressions upon reflecting on the career training experiences that participants discussed in their interviews, and how they addressed the research questions. 
Below are examples of two memos that were recorded on February 23, 2018 (11:17pm)

and April 10, 2018 (10:51pm) respectively.

\section{PATTERN: PAST IS PROLOGUE}

"An interesting recurrence in the interviews with both graduates and current trainees, is the women's interest in entrepreneurship and starting their own businesses. These are personal goals that don't necessarily run counter to the training outcomes of the career training programs, but are complimentary. Many trainees were small business owners prior to resettling in the United States, and are likely drawing from those experiences. They cited that a motivation to participate in the career training programs was to learn the sewing standards for garments in the U.S. apparel industry. This along with the flexibility inherent in business ownership versus working on the factory floor was attractive to participants. However, the lack of small business development know-how left their aspirations as hobbies. This may be an area that textile and apparel NPOs could venture into, because after-all small businesses are the bedrock of the U.S. economy."

\section{PATTERN: REMITTENCES}

"One of the recurring motivators for finding work or working that is mentioned by trainees, is having the ability to send money to relatives that are still in their native countries or living in refugee camps. The trainees felt that they have been given an awesome opportunity in being resettled in the United States, and so almost appear to feel obliged to help those relatives that were left behind. Their unwavering focus on work, actually stagnated their assimilation into this society. They become workhorses. They abandoned the one thing that could propel them to the next level ESL classes. Some of them, ten years after being resettled in the United States, are so proud of all that their families (overseas) have been able to accomplish with their aid but have personally made very little progress in their own lives, and still rely on the state safety net to survive."

Descriptive coding was selected as the coding method because it is the best

method to generate the types of answers needed based on the research questions asked.

Descriptive coding is also sometimes referred to as topic coding. This coding strategy

essentially encapsulates in a word or brief phrase the basic topic of a passage of

qualitative data. It is important to make the distinction that the topic is what is written or 
discussed in the data while the content is the substance of the message. The words or short phrases then become the codes that are later developed in categories and then themes. The use of multiple sources of data that is characteristic of case study research makes descriptive coding ideal for the research design utilized in this study because the coding method can be applied to interview transcripts, field notes, journals, documents, correspondence, physical artifacts, and video (Saldaña, 2014). Interview transcripts were analyzed, and documents such as observational protocols and field notes were used to corroborate the interviews. Field notes and observational protocols document the important physical artifacts and the physical environment of the training world of participants in the study. Thus, everything from the maintenance of the training classroom to the curriculum used in the training program is inference laden. The use of descriptive coding of the qualitative data assists in the documentation of the types of experiences that the graduate and current trainees have during their career transition processes.

\section{Data Collection Timeframe}

Data through direct and participant observations, semi-structured interviews and physical artifacts were collected over a three week period. Archival and documentary data were collected three months before site visits were made and up to six weeks after the completion of the site visits because formal IRB approval to conduct research using adult human subjects through the University of Missouri was not needed. Therefore, research data included a review of company websites, training curriculum (where available), interviews with seven graduate trainees and seven current female trainees with refugee backgrounds, 10 training providers, and the researcher's direct observations to 
enable a holistic understanding of the phenomenon being studied. After each interview with a current trainee, a brief analysis was conducted with the data to check for consistency with the aims of literal replication -did each subsequent interview confirm the evidence gathered in the majority of the earlier interviews (Yin, 1994)? This lead to the interview protocol being slightly revised at this point to reflect more targeted questions. When the researcher analyzed the fifth interview, she sensed that there was no new insight gained. Two more interviews were conducted with current trainees to ascertain that saturation had been attained. When no new information surfaced from these additional interviews, data collection stopped.

The next group of interviews was with graduate trainees. The data from these interviews was used to investigate any graduate-specific factors that hindered or facilitated motivation to participate and complete the training programs, and to confirm or disprove the patterns from earlier interviews with current trainees (replication logic). Similar to the first group of trainees, brief analyses were conducted on data from each interview before proceeding to the next one. When the fifth interview with a graduate trainee did not yield any new information, data collection only proceeded for another two cases. Data collection ceased after the seventh case when the researcher sensed that no new information was being provided through the interviews.

Training providers were interviewed at their convenience, this lasted throughout the three week period. A diagram of the process used in the study of the career transition phenomenon is illustrated in Figure 3. The data were compiled during analysis to strengthen findings as various sources were pooled to promote a better understanding of 
the career transition experiences of women trainees with refugee backgrounds in textile and apparel NPOs and what facilitates or hinders their attainment of training outcomes. 


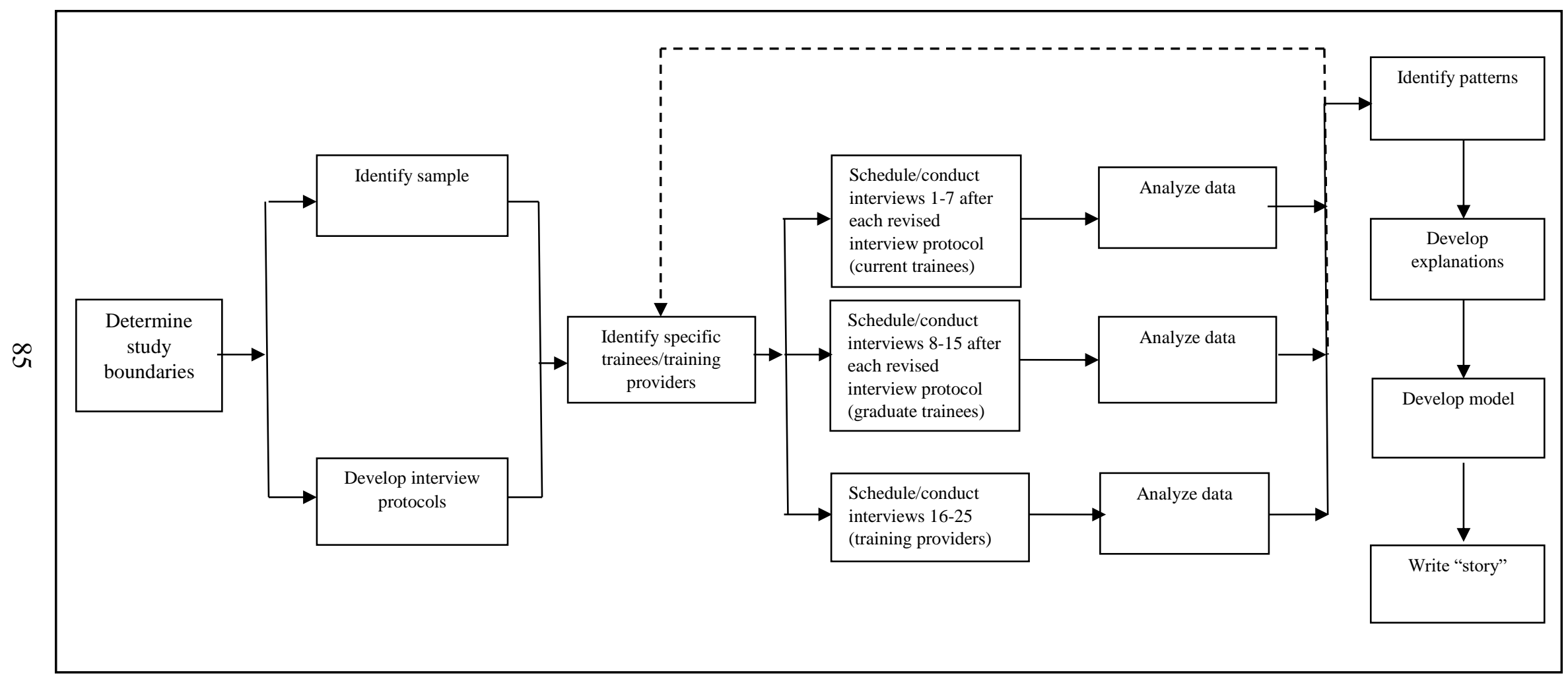

Source: Adapted from Yin, R.K (1994) Case Study Research

Figure 3. Data collection strategy in embedded case study. 


\section{Data Saturation}

Data saturation may be associated with the stage when further collection of data provides little in terms of information or insights in a qualitative research study (Roy et

al., 2015). The concept of data saturation is dependent on the nature of the data source as well as the research question. There is a higher likelihood of reaching data saturation if the data collection is purposeful. The more precise a question, the quicker it tends to reach data saturation. In this study data saturation was ascertained differently with each of the groups sampled. In the case of current trainees, data were briefly analyzed after each interview to check for confirmation of evidence collected from previous interviews. After the fifth interview, the researcher sensed that saturation had been reached, but proceeded to conduct two more interviews with current trainees to make sure that no new information surfaced. When there was no evidence of new information, the researcher concluded interviewing current trainees. However, in the case of graduate trainees, the researcher's aim was to identify any graduate-specific factors that hindered or facilitated motivation to participate and complete the training programs, and to confirm or disprove the patterns from earlier interviews with current trainees (replication logic). After completion of the fifth interview with graduate trainees, it was determined that no new evidence had been obtained. Data collection continued with two more graduate trainees to ascertain that no new information was being uncovered. Training providers were interviewed to provide contextual information about the training programs. The researcher did not reach a sense of saturation with this group of participants until the eighth participant, suggesting the heterogeneity of the group may have contributed to number of interviews it took to reach saturation. Two more interviews were conducted 
after that point to ensure that additional research participants yielded no new insights. Saturation occurred sooner among the cases of trainees with refugee backgrounds than it did among the training providers.

\section{Auditability}

Auditability in qualitative studies is demonstrated when another researcher can clearly follow the decision trail of the researcher and arrive at a similar or comparable conclusion (Creswell, 2009). Therefore, it is important that researchers document both their methods and provide details to all the steps taken (Yin, 2003). Several of the procedures that were used in this study to provide an audit trail included (a) review of interview transcripts to ensure they were free of error after transcription, and (b) codes were diligently compared during the coding process to confirm that definitions of codes remained consistent throughout the process of analysis, (c) keeping a field journal that records the analysis and interpretation done.

\section{Trustworthiness}

Trustworthiness means that the researcher uses prescribed procedures to check for the accuracy of the findings in the study. The trustworthiness of the findings is ascertained from the researcher's, the participant's, and/or the reader's perspectives (Creswell, 2009). In this study, several strategies were used to promote trustworthiness, they included: (a) triangulation of data sources to build a coherent justification of themes; (b) member checking to determine the accuracy of data, themes, interpretations and conclusions by participants (Lincoln \& Guba, 1985; Merriam, 1998); (c) use of rich, thick descriptions to report and convey findings in a more realistic manner; (d) clarification of 
researcher biases throughout the study; and (e) looking for negative cases to provide additional support for the analysts conclusions.

Trustworthiness is also interpreted as the extent to which an account accurately represents the social phenomenon to which it exemplifies (Hammersley, 1992). Constructivists contest the presence of a single truth that exists independent of the researcher and the sample under study. So in essence, researchers cannot ever talk about whether things are accurately represented or not. But rather what needs to be asked is; Do we trust the person who is doing the research to arrive at findings that are useful, insightful etc. In other words, is the account of their (the research participants) career transition experiences accurate?

\section{Transferability}

Qualitative researchers usually do not generalize findings from qualitative inquiry to those outside of the people, sites, or places being studied (Creswell, 2009). "Particularity rather than generalizability is the hallmark of qualitative research." (Creswell, 2009, p. 193). Qualitative research is valued for its ability to produce description and themes developed in the context of a specific site (Creswell, 2009). However, according to Yin (2009) one of the strengths inherent to multiple cases studies is replication logic. In case study research, when researchers replicate a finding through replication in multiple cases, with each case carefully selected because it either predicts similar or conflicting results, the resulting evidence provides for a broader application (Yin, 2009). 


\section{Data Analysis}

An inductive approach was used to analyze the data because of the qualitative nature of the study, using an emergent framework to group data and then look for relationships. The nature of the central research question and the related sub-research questions and the answers sought also influenced the choice in coding strategies made. According to Trede and Higgins (2009), "Research questions embed the values, world view and direction of an inquiry. They also are influential in determining what type of knowledge is going to be generated.” (p. 18). Epistemological research questions address theories of knowing and understanding of the phenomenon of interest which in this case is career transition experiences. These kinds of research questions typically advocate the exploration of participant experiences and perceptions found within the data (Saldaña, 2014).

For each case in this study, data were analyzed at both the individual level and the group level and a within-case analysis ensued. Upon completion of that, a cross-case analysis was conducted between graduate trainees and current trainees and the researcher compared results among the cases. There was no cross-case analysis conducted across the training providers who were interviewed for this study. Data from training providers were analyzed to better inform the context of the cases -skills training programs. This first round of analysis took place at the sub-unit level of cases to reflect the embedded nature of the graduate and trainee cases. The second-round analysis was completed in order to conduct a cross-case comparison between the groups.

The embedded nature of this multiple case study revealed the nested nature of the career transition experiences of women with refugee backgrounds nested within the 
context of the textile and apparel NPO's skills training program. Qualitative data were used to explore each on these levels, and to provide thick description and understanding.

Data analysis began at the single case level for each sub-unit in the embedded case study. Thus, for this research study, qualitative data were first analyzed within each case and at each level (career transition experience and graduate trainee/current trainee) rather than generally across cases (Yin, 1989). A first round of open-coding was used before a second round of more focused purposive coding method was used to analyze the data in this study. As the process continued, each new interview was compared to the previous one for confirming or disconfirming evidence. Codes, categories and then themes were built from the raw data. This process was iterative and included input from participants at given stages (Creswell, 2009). As the interview process proceeded patterns emerged, allowing the researcher to form an early interpretation of how motivation influenced training behavior during the career transition experiences.

The researcher proceeded with data analysis with knowledge of what the literature acknowledged should be present in the data, based on the researcher's past experience with research on career transitions, and a series of analytic memos created from the interview process. Consistent with qualitative inquiry using coding strategies, transcribed interview data were coded and categorized into themes (Saldaña, 2014). This was done by identifying specific phrases in the interview transcripts that were applicable to the research questions that later formed the initial descriptive codes in the analysis. The preliminary themes were reviewed by a methodology expert, who recommended the combination of some of the themes into a larger theme. Once that was done, a codebook was drafted that included the title of each theme, its sub-themes, a description, and low 
inference descriptors. The same process of data analysis was followed for interview transcripts from all three groups of participants in this research study.

\section{Summary}

In summary, this research study explored the career transition experiences of women trainees with refugee backgrounds in textile and apparel NPOs in the United States, describing what facilitates or hinders the motivation of women trainees of refugee backgrounds to participate in career transition programs and attain training outcomes. An embedded multiple case study design was used to provide a detailed description of the trainees' career transition experiences, the implications of which will provide information to future training providers that deliver training programs to people with refugee background. Multiple perspectives were explored through in-depth interviews with training providers, and direct observations by the researcher within the environments that the phenomenon being studied was occurring, offered a deep understanding of not only the training programs and their participants, but also of each organization's vision and mission. 


\section{CHAPTER IV: RESULTS}

Chapter IV presents the (a) description of the sample, (b) thematic categories and interpretations, (c) conceptual model, (d) researcher's reflection, and (e) summary.

The purpose of this study was to gain in-depth understanding of the career transition experiences of women with refugee backgrounds within the context of textile and apparel NPOs in the United States by exploring what motivated them to participate in the training programs and attain training outcomes. Qualitative data were collected from five NPO career transition environments, providing a deeper understanding of the context in which the training occurred. At the individual level, semi-structured interviews were used to gain insight about individual's experiences while participating in the career training programs. A narrative giving the general overview of the results follows, with tables summarizing the characteristics of each textile and apparel NPO's training program, as well as demographic information of each case, and the training providers provided. 
Table 2 .

Characteristics of textile and apparel NPOs' career training programs

\begin{tabular}{|c|c|c|c|c|c|}
\hline $\begin{array}{l}\text { Name of } \\
\text { NPO/train } \\
\text { ing } \\
\text { program } \\
\text { (year } \\
\text { founded) }\end{array}$ & $\begin{array}{l}\text { Avenio/Professional } \\
\text { Industrial Sewing } \\
\text { Program (2013) }\end{array}$ & $\begin{array}{l}\text { Voltan/ Sewing } \\
\text { Class (2017) }\end{array}$ & $\begin{array}{l}\text { Leventis/Sewing Class } \\
\text { (2016) }\end{array}$ & $\begin{array}{l}\text { Univo/ Industrial } \\
\text { Sewing Class (2014) }\end{array}$ & $\begin{array}{l}\text { Salas/Women's } \\
\text { Sewing Class (2015) }\end{array}$ \\
\hline Goal & $\begin{array}{l}\text { - Provide job } \\
\text { readiness classes } \\
\text { - Teach industrial } \\
\text { sewing machine } \\
\text { operation for } \\
\text { apparel industry } \\
\text { production work } \\
\text { - Provide leadership } \\
\text { classes } \\
\text { - Help trainees know } \\
\text { their rights as } \\
\text { workers } \\
\text { - Qualify trainees for } \\
\text { Apprenticeship } \\
\text { Program that's } \\
\text { authorized by US } \\
\text { Department of } \\
\text { Labor }\end{array}$ & $\begin{array}{l}\text { - Encourage women } \\
\text { with refugee } \\
\text { backgrounds to } \\
\text { build friendships } \\
\text { in their } \\
\text { community and } \\
\text { avoid isolation } \\
\text { - Teach sewing } \\
\text { skills and general } \\
\text { sewing machine } \\
\text { operation apparel } \\
\text { industry work } \\
\text { - Provide time for } \\
\text { English language } \\
\text { practice }\end{array}$ & $\begin{array}{l}\text { - Teach artisans } \\
\text { repurposing and } \\
\text { alteration skills } \\
\text { - Teach how to repair, } \\
\text { re-fit \& repurpose } \\
\text { common household } \\
\text { textile goods and } \\
\text { clothing } \\
\text { - Demonstrate how to } \\
\text { work with customers } \\
\text { - Support local } \\
\text { businesses/ artisans } \\
\text { by providing skilled } \\
\text { affordable labor } \\
\text { - Support goal to } \\
\text { educate public on } \\
\text { sustainable practices } \\
\text { (keep textiles out of } \\
\text { landfills) }\end{array}$ & $\begin{array}{l}\text { - Provide trainees } \\
\text { with hands-on } \\
\text { sewing training } \\
\text { - Develop trainee } \\
\text { confidence in } \\
\text { speaking English } \\
\text { - Provide trainees } \\
\text { with exposure to a } \\
\text { workplace setting } \\
\text { - Complete } \\
\text { assignments that } \\
\text { assist trainees } \\
\text { build a portfolio } \\
\text { that demonstrates } \\
\text { their skills to } \\
\text { potential } \\
\text { employers }\end{array}$ & $\begin{array}{l}\text { - Encourage women } \\
\text { with refugee } \\
\text { backgrounds to } \\
\text { participate in } \\
\text { classes to improve } \\
\text { mental health, avoid } \\
\text { isolation, and } \\
\text { engage in } \\
\text { community relation } \\
\text { building } \\
\text { - Teach trainees } \\
\text { introductory to } \\
\text { intermediate level } \\
\text { sewing skills }\end{array}$ \\
\hline
\end{tabular}




\begin{tabular}{|c|c|c|c|c|c|}
\hline $\begin{array}{l}\text { Name of } \\
\text { NPO/train } \\
\text { ing } \\
\text { program } \\
\text { (year } \\
\text { founded) }\end{array}$ & $\begin{array}{l}\text { Avenio/Professional } \\
\text { Industrial Sewing } \\
\text { Program (2013) }\end{array}$ & $\begin{array}{l}\text { Voltan/ Sewing } \\
\text { Class (2017) }\end{array}$ & $\begin{array}{l}\text { Leventis/Sewing Class } \\
\text { (2016) }\end{array}$ & $\begin{array}{l}\text { Univo/ Industrial } \\
\text { Sewing Class (2014) }\end{array}$ & $\begin{array}{l}\text { Salas/Women's } \\
\text { Sewing Class (2015) }\end{array}$ \\
\hline $\begin{array}{l}\text { Target } \\
\text { group }\end{array}$ & $\begin{array}{l}\text { Men and women } \\
\text { with refugee and } \\
\text { immigrant } \\
\text { backgrounds }\end{array}$ & $\begin{array}{l}\text { Eight Afghani } \\
\text { women with } \\
\text { refugee } \\
\text { backgrounds }\end{array}$ & Public housing residents & $\begin{array}{l}\text { Public housing } \\
\text { residents }\end{array}$ & $\begin{array}{l}\text { Somali women with } \\
\text { refugee backgrounds } \\
\text { living in the } \\
\text { community near Salas }\end{array}$ \\
\hline $\begin{array}{l}\text { Program } \\
\text { Duration }\end{array}$ & $\begin{array}{l}\text { - } 8 \text { weeks (offered } \\
\text { every quarter) } \\
\text { - Structured program } \\
\text { - } 12 \text { hours a week }\end{array}$ & $\begin{array}{l}\text { - } 6 \text { weeks } \\
\text { - } \text { Structured } \\
\text { program } \\
\text { - } 6 \text { hours a week }\end{array}$ & $\begin{array}{l}\text { - } 9 \text { months } \\
\text { - } \text { Self-directed } \\
\text { program } \\
\text { - } 3 \text { hours a week }\end{array}$ & 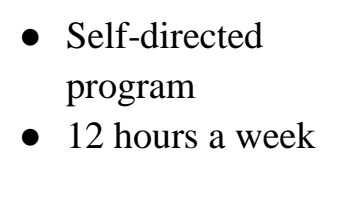 & $\begin{array}{l}\text { - } \text { Self-directed } \\
\text { program } \\
\text { - } 4 \text { hours a week }\end{array}$ \\
\hline $\begin{array}{l}\text { Proof of } \\
\text { Training }\end{array}$ & $\begin{array}{l}\text { - Certificate of } \\
\text { completion }\end{array}$ & - N/A & $\begin{array}{l}\text { - Certificate of } \\
\text { completion }\end{array}$ & - N/A & - N/A \\
\hline $\begin{array}{l}\text { English } \\
\text { Language } \\
\text { Learning }\end{array}$ & - Yes & - Yes & - No & - No & - No \\
\hline
\end{tabular}




\begin{tabular}{|c|c|c|c|c|c|}
\hline $\begin{array}{l}\text { Name of } \\
\text { NPO/train } \\
\text { ing } \\
\text { program } \\
\text { (year } \\
\text { founded) }\end{array}$ & $\begin{array}{l}\text { Avenio/Professional } \\
\text { Industrial Sewing } \\
\text { Program (2013) }\end{array}$ & $\begin{array}{l}\text { Voltan/ Sewing } \\
\text { Class (2017) }\end{array}$ & $\begin{array}{l}\text { Leventis/Sewing Class } \\
\text { (2016) }\end{array}$ & $\begin{array}{l}\text { Univo/ Industrial } \\
\text { Sewing Class (2014) }\end{array}$ & $\begin{array}{l}\text { Salas/Women's } \\
\text { Sewing Class (2015) }\end{array}$ \\
\hline $\begin{array}{l}\text { Number } \\
\text { Volunteers } \\
\text { per class } \\
\text { (not } \\
\text { including } \\
\text { the } \\
\text { instructor) }\end{array}$ & - None & - Four & - One & - One & - Two-four \\
\hline Partners & $\begin{array}{l}\text { - Fair Work Center } \\
\text { - The Maker } \\
\text { Coalition } \\
\text { - US Department of } \\
\text { Labor } \\
\text { - Goodwill Industries }\end{array}$ & - N/A & $\begin{array}{l}\text { - Seattle Housing } \\
\text { Authority } \\
\text { - The Maker Coalition } \\
\text { - US Department of } \\
\text { Labor }\end{array}$ & - N/A & - N/A \\
\hline
\end{tabular}

Note. ${ }^{1}$ All career training programs were in operation within 3 months of data collection.

Sources: This table emerged from the interview data in the study. 
What follows is a descriptive summary of each of the five data collection sites, the main characteristics of the career training program offered, and the women trainees and training providers that participated in this research study. All the names of organizations and women who were part of this study were replaced with pseudo names to maintain confidentiality. From this point forward, all information presented in tables and figures emerged from the data collected in this research study.

\section{Avenio}

Avenio, was a textile and apparel NPO founded by two immigrant women in 2009 (Claire, personal communication, November 9, 2017). Their mission was to provide training to apparel product developers as a way of empowerment (Claire, personal communication, November 9, 2017). Avenio provided training to refugees and immigrants from all over the world and worked to help program graduates secure local, fair paying jobs to apply their newly acquired knowledge and skills to. Many of the refugees Avenio trains had previous sewing experience and seek careers that will use these sewing skills.

The organization provided training activities that sought to prepare refugees for employment with local mainstream employers in the apparel industry. Avenio worked in close partnership with local businesses and other NPOs to design and fund the programming it offered to its trainees. Avenio also relied on donations and grants to fund its activities (Claire, personal communication, November 9, 2017). The organization offered a variety of training programs customized to equip adult learners and non-native English speakers with the skills local businesses needed to operate profitably and sustainably so as to strengthen the local economy and create a thriving community. These 
included industrial sewing programs, internships, pop-up events, upscaling and repair workshops, and freelance studio. Avenio's physical location was in an industrial business park.

Avenio was an active community-based organization with a bevy of programs focused on empowering people of immigrant and refugee backgrounds through skill development. However, by the time the researcher visited the organization, the career transition program that focused on sewing skills was suspended for three months due to a lack of funding. As such, there were no current trainees. However, artifact examination was conducted in the studio space where the training program took place. Artifacts provided a general view of the training program's activities (Yin, 2014), and were listed on the observational protocol. In this study, artifacts included production equipment, training facilities, posters and materials physically displayed throughout the studio space. All were inventoried on an observational protocol worksheet.

Using the interview protocols developed, seven research participants were interviewed that were affiliated with this textile and apparel NPO. Training providers were served as interviewees, and as key informants in recommending potential participants among their training program graduates. Training providers recommended potential programs graduates by sending an email out to the group and briefly described the study. Those graduates that responded to the email and indicated interest in the study were forwarded to the researcher, along with an informal introduction. From that point on the researcher was responsible for follow-up and setting appointment times with each graduate. Not all program graduates who expressed interest in participating in the study could participate because they didn't all meet the criteria set forth for all study 
participants. Table 3 details the role and location chosen by the participant for the scheduled semi-structured interviews.

Table 3.

Semi-structured interviews at Avenio's Training Program

\begin{tabular}{lll}
\hline Participant & Role in NPO & Interview Location \\
\hline Rayna & Board Director & Phone \\
Claire & Co-founder & Phone \\
Beth & Instructor & Avenio \\
Anita & Graduate Trainee & Phone \\
Jennifer & Graduate Trainee & Phone \\
Ruth & Graduate Trainee & Local Community College \\
Rose & Graduate Trainee & Phone
\end{tabular}

Note. ${ }^{1}$ All participants' names are pseudo names.

\section{Univo}

Univo offered a sewing skills training program that qualified as a source of research participants for this study. This program was a collaboration with Avenio. The career transition program was created with the purpose of offering creative clothing repair and alteration workshops to more advanced sewers as a way to expand their sewing skills to include professional techniques for re-imagining clothing. Once trainees had attended and achieved proficiency in operating a sewing machine, they had the ability to take rotating workshops on repair, alterations, and upcycling. During each workshop instructors demonstrated professional sewing techniques and trainees had the opportunity to bring garments from home to share with the class and re-purpose them. They were then taught how to alter the clothing as well as creative, durable ways to repair and reconstruct clothing to extend its use. Univo supported the city's public utilities' goal to educate the 
public on sustainable practices by keeping textiles out of landfills (Carly, personal communication, November 10, 2017). Training sessions were held once a week for a period of three hours.

Once a month, training providers from this program also directed pop-up sewing events in various locations within public housing properties in the city limits, as a form of community-outreach as well as a way to demonstrate to trainees how to give back to their community. The purpose of these pop-up sewing events was to promote slow fashion (Eve, personal communication, November 10, 2017). They were held in communities where the training providers saw a need for the skills, and sewing machines and other sewing materials were brought to the events, and sewing instruction and assistance was provided to event attendants by training providers and trainees from Univo (Carly, personal communication, November 10, 2017).

The researcher used participant observations, in-depth semi-structured interviews, and examined documents and physical artifacts as data sourced from Univo's career transition program. Most of the data collection took place in Univo's community room which is in a public housing residential building. Data collection occurred during the program's weekly three hour session for participant trainees. The facility was a multipurpose room that had tables and chairs that could be placed in any desired configuration, with a kitchenette off to one side of the room. Training providers arrived early and the researcher introduced herself and proceeded to assist them with removing ten singer home sewing machines, materials and notions from the nearby storage cage and rolling them to the multipurpose classroom. Participant observations were made for the duration of the three hour class. 
Instructors introduced the researcher as well as the purpose of the researcher's presence in the classroom to the trainees and then the researcher was allowed to walk around the classroom observing the trainees as they worked, asking them questions, as well as answering questions related to their projects. During this time, the researcher filled out a detailed observational protocol worksheet for the site, being careful to record note-worthy behaviors, and artifacts of interest. The researcher was also given the chance to present her own work on African textiles to the group, so as to quickly "fit in" to the environment.

Subsequently, both the training providers and the researcher invited individual trainees to participate in the research study. Those that were interested gave their names and phone numbers to the researcher so an interview time could be arranged at a mutually convenient time. Training providers also recommended additional participants to the researcher, which the researcher approached at the end of the training session. At the conclusion of the weekly program, in-depth interviews were conducted with all training providers that were on-site. Follow-up calls to the selected trainees yielded additional interview data. Participant interviews lasted approximately an hour and were conversational in nature. Table 4 details the roles and scheduled location of the interview chosen by the participant for the semi-structured interview.

Training providers furnished documentary information in the form of program flyers, training curriculum and an example of a weekly reflection template to the researcher, for research purposes. 
Table 4.

Semi-structured interviews at Univo's Training Program

\begin{tabular}{lll}
\hline Participant & Role in NPO & Interview location \\
\hline Eve & Community Outreach/Artisan & Univo's community room \\
Raven & Lead Artisan & Univo's community room \\
Carly & Program Coordinator & Univo's community room \\
May & Current Trainee & Phone \\
Justine & Current Trainee & Phone \\
Kelly & Current Trainee & Phone \\
Roxanne & Current Trainee & Phone \\
\hline
\end{tabular}

Note. ${ }^{1}$ All participants' names are pseudo names.

\section{Leventis}

Leventis' sewing skill's training program was taught at its community center which was located in the heart of the city's downtown area. The program was held in a large multi-purpose room with a storage room where all sewing-related machinery, material and notions were kept in-between classes. Although this career transition program was called an industrial sewing class, the organization's website featured images of women sewing on singer home sewing machines. Students in the class were encouraged to practice English by communicating with each other in English. Only residents of Univo's public housing community were permitted to participate in the classes.

During the site visit, Rita, the substitute instructor introduced the researcher at the end of the class and therefore the researcher was only able to conduct direct observations during the length of the three hour class and note observations on an observational protocol worksheet. Direct observations are made of the spaces where the training 
program occurs as well as where work tasks are undertaken, and the researcher can record information as it occurs. In this instance, the researcher although assuming a nonparticipant role, looked for certain behaviors that provided insight to the research questions. Non-participant observations gave the researcher first-hand experience with each participant and enabled the researcher to observe anything that was unique and unusual taking place (Creswell, 2009). Interviews with three current program trainees were conducted during the course of the class. Class attendance was low. On Rita's recommendation, the researcher reached out to two absentee trainees because they frequently attended class, but was unable to set up an interview with either one. Table 5 details the roles and the locations chosen by the participants for the semi-structured interview.

Table 5.

Semi-structured interviews at Leventis’ Training Program

\begin{tabular}{lll}
\hline Participant & Role in NPO & Scheduled interview location \\
\hline Tammy & Teaching Assistant & Leventis Community Center \\
Erin & Graduate Trainee & Leventis Community Center \\
Rebecca & Current Trainee & Leventis Community Center
\end{tabular}

Note. ${ }^{1}$ All participants' names are pseudo names.

\section{Voltan}

Founded in the late 1970s, Voltan had grown to become the largest voluntary resettlement agency (VOLAG) in the western part of the state of Washington, resettling over 1100 refugees into Seattle in 2016 (Haley, personal communication, November 9, 2017). Its mission was to create opportunities and partnerships with the local church in assisting with the settlement of refugees and immigrants from over 50 countries to the 
area (Haley, personal communication, November 9, 2017). Voltan’s many programs provided much of the traditional support typically offered to refugees. Such as, refugee resettlement services to provide the basic needs of refugee families as they start a new life in the area, on-site English language classes for refugees to acquire important language skills, and immigrant legal services to assist former refugees navigate the legal processes to obtain citizenship (Haley, personal communication, November 9, 2017).

Voltan had developed innovative programs related to employment and resiliency that help refugees beyond the initial resettlement process. One such program was the sewing skill training program targeted to Asian women with refugee backgrounds. The six week program met for six hours each week at a local church. The program focused on providing a space where women could build friendships with other women in their community, learn how to sew, and practice their English-speaking skills. Childcare was provided to the trainees' children in a room right next to the sewing classroom. Semistructured interviews were conducted with four participants at Voltan's main offices prior to a tour of the classroom facility at the nearby church. Although, the researcher planned to conduct all interviews in English, one of the graduate trainees interviewed spoke little conversational English and so, the second graduate trainee provided translation during the interview. The translator had helped the U.S. military with translation in her country of origin, and so she was able to understand the researcher's questions and translate them to the second interviewee, as well as translate the second interviewee's answers for the researcher. Table 6 details the role and the scheduled location chosen by the participant for the semi-structured interview. 
At the time of the researcher's visit, one training session had just ended and Voltan had not started a new one yet. The researcher documented what filled the classroom space in detail using an observational protocol, during the tour of the church. Artifacts included apparel production equipment, furniture, class hand-outs, posters, and sewing notions and materials.

Table 6.

Semi-structured interviews at Voltan's Training Program

\begin{tabular}{lll}
\hline Participant & Role in NPO & Interview location \\
\hline Haley & Program Coordinator & Voltan's offices \\
Danielle & Program Coordinator & Voltan's offices \\
Lauren & Graduate trainee & Voltan's offices \\
Judy & Graduate trainee & Voltan's offices
\end{tabular}

Note. ${ }^{1}$ All participants' names are pseudo names.

\section{Salas}

Salas' sewing skills training program was a project initiated by a local refugee safety task force with the purpose of reducing isolation and empowering East African women of refugee backgrounds, by promoting resiliency through community engagement and education (Rita, personal communication, November 11, 2017). The task force was formed when the results of a focus group with East African women of refugee backgrounds in the community indicated that the women wanted to learn how to sew. So the task force raised funds to purchase sewing equipment and found volunteers to provide sewing instruction. The purpose of this 10 -week training program was to provide the trainees with the skills needed to pave the way to a career in sewing. 
Participant observations were made throughout one four hour training session, and the researcher took descriptive notes such as the type of individual observed, observed behaviors, reflexive notes such as the researcher's feelings or impressions, and demographic notes that addressed the date, time and place the observation occurred in. During this training session the training provider introduced the researcher at the end, and so the researcher had to take the initiative during the session to introduce herself and the reason she was there to the trainees in order to build rapport. At the recommendation of the training provider four trainees were invited to participate in the study at the end of the training session, but the researcher only successfully followed-though with two of them. Semi-structured interviews were conducted on site and over the phone. Table 7 details the role and the scheduled location chosen by the participant for the semi-structured interview.

Table 7.

Semi-structured interviews at Salas' Training Program

\begin{tabular}{lll}
\hline Participant & Role & Scheduled interview location \\
\hline Rita & Instructor & Local Community Center \\
Natali & Current Trainee & Local Community Center \\
Emma & Current Trainee & Local Community Center
\end{tabular}

Note. ${ }^{1}$ All participants' names are pseudo names.

\section{Description of the Sample}

The sample was comprised of 24 in-depth interviews with women consisting of:

(a) seven current trainees, (b) seven graduate trainees, and (c) 10 training providers. 
Table 8 presents the demographic characteristics of the current and graduate women trainees with refugee backgrounds.

Table 8 .

Demographic Characteristics of Trainees with Refugee Backgrounds

\begin{tabular}{clr}
\hline Characteristic & Current Trainees & Gra \\
\hline Age & & 3 \\
$18-27$ & 2 & 2 \\
$28-37$ & 0 & 1 \\
$38-47$ & 2 & 1 \\
$48-57$ & 1 & 0 \\
$58-67$ & 2 &
\end{tabular}

Graduate Trainees

3

2

1

1

Race

Black or African American

Asian

Native Hawaiian or Pacific Islander

6

0

1

Marital Status

Single

Married

Divorced

Widower

Engaged

2

2

3

1

1

0

1

5

1

Trainee with Children under the age of 7 years

Yes

2

No

Country of Origin (COO)

Afghanistan

Burma

Cambodia

Democratic Republic of Congo

Eritrea

Ethiopia

Philippines

Somalia

0

0

0

0

1

1

1

4

1

0

Apparel-related Experience in $\mathrm{COO}$

Design/Sewing

Retail

No Experience

3

1

3

6

0

1

Number of languages spoken (excluding

English)

$1-2$

3-4

5-6

3

3

1

3

2

2

Highest level of formal educational earned 


\begin{tabular}{lll}
\hline Characteristic & Current Trainees & Graduate Trainees \\
\hline None & 1 & 0 \\
Vocational certificate & 0 & 0 \\
Some High School & 1 & 1 \\
High School Diploma & 2 & 4 \\
Associates degree & 1 & 2 \\
Bachelor's degree & 1 & 0 \\
English on arrival to the United States & & \\
Yes & 2 & 2 \\
No & 5 & 5 \\
& & \\
Current Occupation & & 1 \\
Homemaker & 5 & 1 \\
Student & 1 & 1 \\
Embroidery artisan & 0 & 1 \\
Bundle work & 0 & 1 \\
Custom garment orders & 0 & 1 \\
Food Service & 0 & 0 \\
Daycare worker & 1 & 1 \\
Unemployed & 0 & \\
& & 4 \\
Years lived in United States & & 1 \\
$1-5$ & 1 & 1 \\
$6-10$ & 3 & 0 \\
$11-15$ & 1 & \\
$16-20$ & 1 & \\
$21+$ & 1 & \\
& & \\
\hline
\end{tabular}

Demographics of Current Trainees. The current trainees were aged between 25 years and 65 years, and had been living in the United States from one year to 22 years. Three out of seven participants were married, and the remaining four women were either single, divorced or widowed. Most of the trainees (five out of seven) were homemakers, one was a student and the other worked at a daycare center. Only two participants interviewed in this group had children under the age of seven. Educational background of the participants was varied, with only one participant not having a formal education, and all others having at least some high school education. The highest level of education achieved among this group of participants was a Bachelor's degree. Although only two out of seven trainees spoke English on arrival to the United States, their ability to learn 
languages other than their native languages was evident by the number of languages they could speak. Somalia was the source country with the highest number of trainees interviewed, followed by Eritrea, Ethiopia, and the Philippines. Four out of seven trainees had apparel-related experience prior to resettling in the U.S. Apparel-related work experience comprised of designing, sewing, and selling garments and textiles, or the ownership of a clothing retail shop.

Demographics of Graduate Trainees. Graduate trainees were, on the whole, younger than current trainees, with the majority of trainees (six out seven) in their twenties and thirties and the oldest graduate trainee being 51 years old. Graduate trainees also reported living in the United States an average of five fewer years than current trainees. Although the majority (six out seven) of the graduate trainees were refugees from Asian countries, there was greater diversity in their countries of origin in comparison to their counterparts. Graduate trainees were natives of five Asian countries (Afghanistan, Burma, Cambodia, The Philippines, and Vietnam) and one African country (Democratic Republic of Congo).

There were fewer married women among the group of graduate trainees with a total of four married women out of seven, with the remaining women being single, divorced, or engaged. One of the graduate trainees was a homemaker. Three of the graduate trainees interviewed worked in the apparel industry either on contract work (bundle work) or as artisans. The rest of the graduate trainees were either in school, employed in food service, or unemployed. Educational background of the participants was varied, with only one participant not having a formal education, and all others having at least some high school. The highest level of education achieved among this group of 
participants was a Bachelor's degree. Apparel-related experience was more prevalent among graduate trainees than it was among the group of current trainees. With six out of seven graduate trainees citing fashion-related experience from formal training, sewing for clients, and managing retail shops, during their interviews. Numbers of participants with children under the age of seven years were the same in both groups of trainees, as was the number of participants who spoke English on arrival to the United States.

Demographics of Training Providers. Table 9 presents the demographic characteristics of the training providers. All 10 training providers worked in areas of the textile and apparel NPOs in capacities that either provided trainees instruction or support in the way of job leads, or understood the career transition programs that refugees participated in.

Table 9.

Demographic Characteristics of Training Providers

\begin{tabular}{ll}
\hline Characteristic & $\mathrm{n}$ \\
\hline Country of Origin (COO) & 10 \\
$\quad$ USA & \\
Gender & 10 \\
$\quad$ Female & \\
Race & 6 \\
$\quad$ Caucasian & 4 \\
$\quad$ Asian & \\
Number of languages spoken (excluding English) & 3 \\
1 & 7 \\
2 & \\
Formal Apparel-related education earned & \\
$\quad$ None & 4 \\
Vocational certificate & 2 \\
Associates degree & 2 \\
Bachelor's degree & 1 \\
Master's degree & 1 \\
& \\
Apparel-related Training/Experience & 6 \\
Design/Sewing & 4 \\
$\quad$ No Experience &
\end{tabular}




\begin{tabular}{ll}
\hline Characteristic & $\mathrm{n}$ \\
Employment Status & \\
$\quad$ Volunteer & 4 \\
$\quad$ Paid & 6 \\
& \\
Number of years with current textile and apparel NPO & \\
$>1$ & 1 \\
$1-3$ & 7 \\
$4-5$ & 2
\end{tabular}

All ten training providers were American women, with six out of ten of them identifying as Caucasian while the remaining four were Asian. Spanish and English, Vietnamese and English, or Korean and English were the language pairings spoken by seven of the ten training providers. Only three of them spoke just the English language. Most of the training providers had formal apparel-related education and experience that they used in different capacities in their roles within the textile and apparel NPOs, as either paid workers or volunteers. Evidently, the training providers that held paid positions within the textile and apparel NPOs exhibited more interest in the future success of the trainees in their programs, versus those training providers that were volunteers. An example of the way the training providers' used their apparel-related experience was, in helping trainees find apparel production work with local apparel manufactures. Some training providers also assisted trainees in negotiating their contracts for bundle work. Seven of the ten training providers had spent one to three years in their current roles; only one participant revealed that she been working for less than a year in the textile and apparel NPO.

\section{Thematic Categories and Interpretations}

Transcripts from all interviews conducted with trainees and training providers were analyzed. The goal of the qualitative data analysis was to reduce data without losing 
its meaning. During this process it was also important to establish the connection between data collected and how they addressed the research questions, so that research findings are reflective of the data collected in the study. The analysis of the transcribed interviews yielded nine themes. The first theme described how women trainees of refugee backgrounds navigated the five different career training programs and what their outcomes were. This first theme addressed both research questions. This theme was labelled informal pathways of training programs. The next four themes addressed research questions one -how motivation was facilitated among the trainees in the training program. These themes were: (a) building foundations for a new life, (b) creating a sense of community, (c) restructuring family and friend relationships to navigate a new world, and (d) translating skills and knowledge into a new world. The last four themes addressed research question two -how motivation was hindered among the trainees in the training programs. These themes were: (a) physical constraints and language barriers, (b) childcare, (c) unmet expectations, and (d) learned dependency.

\section{Informal pathways of training programs}

The five career training programs were each established with formal training outcomes. Avenio, Leventis and Univo established their training programs with the goal of providing job readiness training for sewing machine operator work in the apparel industry. Volton and Salas training programs focused their training goals on creating community among women of specific ethnic backgrounds; encouraging them out of isolation into a environment whereby they can build friendships with other women, while learning how to sew. After the analysis of interviews with the participants in this study, an informal network between the textile and apparel NPOs emerged. Figure 4 provides a 
visual illustration of the informal pathways to training outcomes as described by participants. This model emerged from the data in the study.

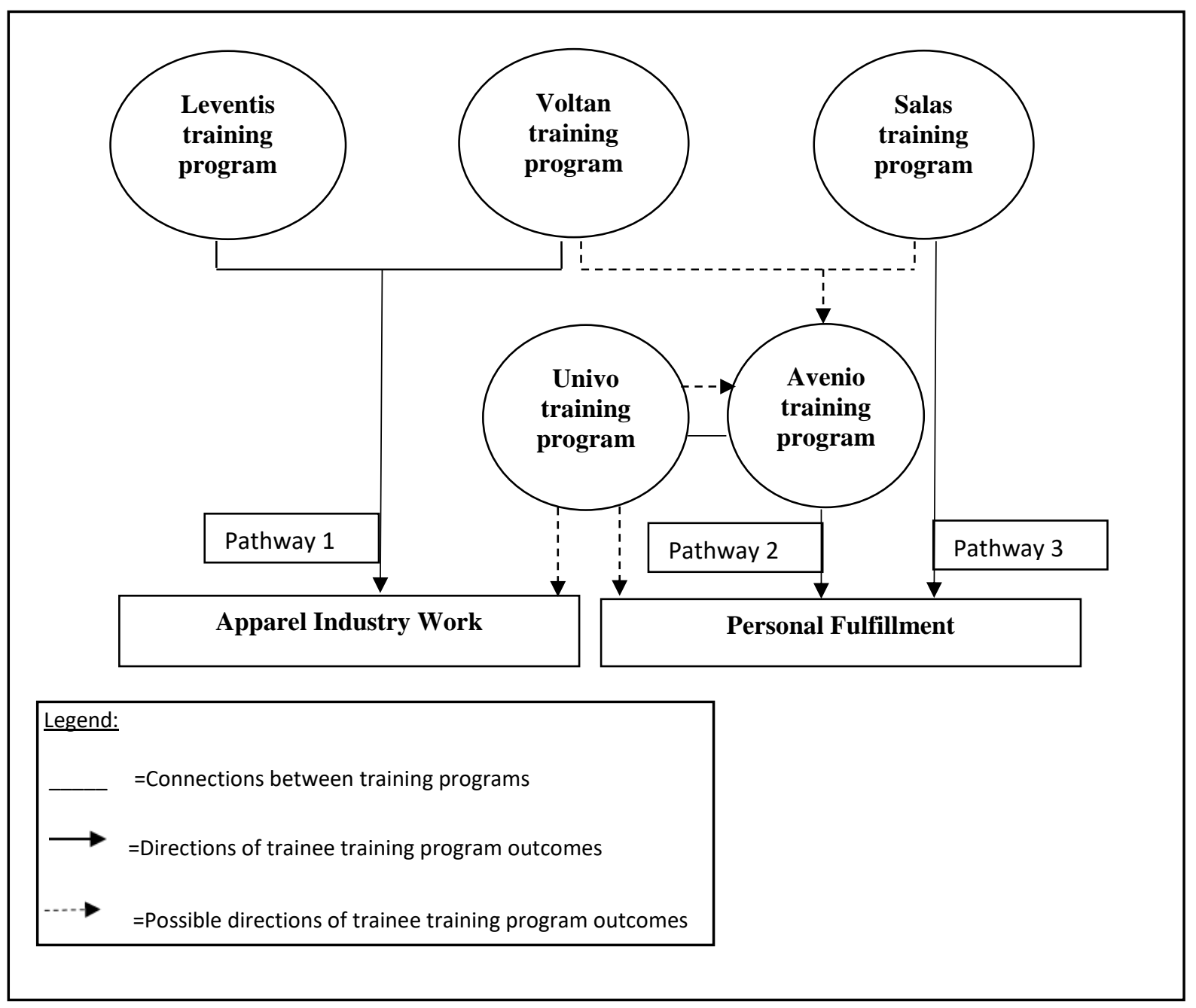

Figure 4. Informal pathways to training outcomes

Pathway 1: Training lead to apparel-related jobs. The training goals of both Leventis and Voltan training program were for their trainees to acquire the appropriate apparel construction skills so that they could work in the apparel industry. Therefore, program trainees and graduates from these two programs were focused on becoming proficient at sewing to receive contracts to do bundle work and custom work. Three out of the four trainees interviewed were graduates from these two programs and indicated 
that they were already recipients of such contracts. The fourth trainee was a current trainee but already anticipating that her future work would be in the apparel industry.

Training providers in these two training programs were very instrumental in assisting graduate trainees: (a) negotiate their work contracts, (b) buy their first home sewing machine, and (c) if graduates had a contract to do bundle work, they would help them secure a loan to buy their first industrial sewing machine. However, to be able to be considered for bundle work and other custom work trainees needed to have displayed mastery of sewing skills at the completion of their training program. The need to secure a loan in order to by the appropriate industrial sewing machine, created a barrier to access work opportunities that graduate trainees had to overcome. For some graduates this barrier proved insurmountable and one that they had not considered prior to enrolling in the training program. Upon reflection, Ruth a graduate trainee from Avenio's training program, articulated this as one of the reasons why she couldn't secure contracts for bundle work despite showing great promise during training.

Ruth|Avenio|Graduate trainee: It did not work because in order to work at home I need to have the industrial machine. This is the first thing I didn't think about that. Yeah, I didn't think about that one.

Pathway 2: Training lead to advanced training with Avenio. At the center of this informal network created by all the training programs cited was Avenio's training program and curriculum. Avenio’s program goal was to have all its graduate secure apparel industry jobs. All training programs in this study utilized Avenio's curriculum to some degree. Rita, an instructor in the Salas training program echoed this fact during her interview.

Rita|Salas|Training provider: I never completed an apparel design program or anything like that. I've just been a home sewer, but I have 
learned from Rayna, with her curriculum. So I just took her curriculum in here and that's been just a fabulous collaboration. Rayna and I have had. And so I try to, um, find women who I think have this skill and have the desire and then I, I, after I've worked with them, then I refer them to Rayna. Um, and I teach to the curriculum but what I've done is I've modified it to, because sometimes a, well, Rayna will have these, um, these samples cut and it takes time to cut all those samples. And put them all together. So when I'm teaching a skill like doing a neck line, sometimes I'll break it down even further.

Avenio's training program was considered more advanced by other training providers in this network because its instruction was structured, and centered on machinery and production flow methods more representative of those found in industry production work. To begin with, the inventory list of Avenio's equipment on the researcher's observational protocol consisted of industrial sewing machines and industrial sergers only, while inventories of the sewing equipment available in the other training programs featured only home sewing machines and sergers. Avenio’s instructors incorporated group exercises into the training program that mimicked the production of garments on a factory floor. Avenio also scheduled field trips for each training group to apparel manufacturing factories so that students started to connect their training to real-world applications. None of the other training programs provided such opportunities. Rayna, an Avenio training provider, pointed out the dual purposes of this practice.

Rayna|Avenio|Training provider: Early in the program we arrange factory visits for our students. This is a double-edge sword, because for trainees this is the first time they have set foot in a factory -and they are shocked! [...] This is not what they expected. This practice has served as a way to weed out of the program, those who are not genuinely interested or do not believe that they have what it takes to eventually work in apparel manufacturing.

Factory visits were not the only way Avenio training providers helped trainees familiarize with their future work environment. During regular training sessions, Avenio training 
providers used a white board to incorporate specific English language vocabulary related to the day's lessons in order to familiarize trainees with industry jargon.

However, of the four Avenio graduates interviewed, none had secured apparel industry work and were using the skills they had learned to sew for their family and friends. Interestingly, three of the four current trainees at Univo, indicated that once their year-long training at Univo was completed they would seek out more advanced sewing training through Avenio.

Pathway 3: Training lead to personal fulfillment. Acquiring skills to fulfill personal goals was a reason some trainees participated in sewing training programs. One out of the four trainees interviewed at Univo indicated that she was participating in the training so that she could sew for her family, and "save" the family some of the cost associated with taking clothes to a seamstress for repair or alteration. The two trainees from Salas also expressed similar sentiments. The structure of Salas' and Univo's training programs were informally organized, the programs were self-directed like the others in this study, and training outcomes were less obvious to trainees. The approach used by training providers was less focused on developing proper industry production techniques and more aimed at trainees acquiring general apparel assembly techniques. Project work also varied widely and trainees were allowed to work on personal projects, as there weren't any clearly established weekly objectives. According to Rita, the lead instructor at Salas, graduate trainees who "showed skill proficiency and who had the desire [to advance their sewing skills]" were referred to Avenio's training program by her. 
Univo's training program was the youngest program out of the five sited in this study. It had been in session for three months when these interviews and observations were conducted. No graduates had been produced by this program yet, thus in Figure 4 there are three possible directions that program graduates could follow: (a) be recommended for advanced training in Avenio's training program, (b) use the skills they had already acquired and find a job in the apparel industry, or (c) turn the skills they had acquired into a hobby by sewing for family and friends.

\section{Building Foundations for a New Life}

The pursuit of a career transition for the women with refugee backgrounds required the ability to wade through information on other training opportunities and hone in on a training program that addressed their unique needs. Sometimes, their social workers played a part in their decision-making process by working to match a woman's interests with an appropriate training opportunity. The needs of the women with refugee backgrounds centered on: (a) the location of the training program being in an easily accessible place relative to their homes, and (b) the childcare needs for their young children being resolved before they would contemplate participating in a training program. This is clear in a statement made by Justine, a 38 year old mother of four, "We are just close by, you know. I can leave the kids and go back and check on them." By participating in a training program, participants were building foundations for their new lives in the United States. They described their participation as a way to "benefit," "be proud of myself," "get better," and "improve my knowledge" through the development of their sewing skills. This process was often driven by the realization that applying their learned skills in a formal or informal work capacity could result in success. Their 
definitions of success sometimes matched those of the training program goals and other times they didn't. Success was described by trainees as "going to the factories or working from home," "the job," "I can fix things and I can sew," "get some knowledge," "if neighbors want me to sew I can do that," and "I can teach others." Growing selfconfident as their skills advanced, drove the self-efficacy in trainees to levels where they believed they could succeed in whatever direction they wished to go. Buckingham et al., (2006) found that mothers of low educational attainment were more confident to pursue other things that they might want after completing their training program. This may explain what happened in the case of these trainees too, because the majority of them had high school diplomas with only a couple of trainees having completed an Associate's or Bachelor's degree.

Roxanne, a 49 year old single mom participating in Univo's training program had a different motive to participating in the training program. As a victim of domestic abuse, it was evident that her being subjected to that trauma for over a decade had crushed her self-esteem and self-image. She admitted that she was "severely depressed," and that "I want to get out and do things, but my body won't move." She used adjectives such as "horrible," "ugly," "so big," and "difficult" to describe her body. However, as she continued to describe her progress in the class with excitement, glimpses of her formerself occasionally reappeared. Community-based interventions for refugee children who have experienced trauma, in the form of creative expression (e.g. drama, art, and creative play), have improved the overall mental health outcomes of children (Miller \& Billings, 1994; Tol, Komproe, Susanty, Jordans, Macy, \& de Jong, 2008). Thus, the signs of change in Roxanne's outlook may be a benefit of her participation in the sewing program 
that is a creative outlet. The excitement she showed in what she had learned and achieved through the program was illustrated when she discussed the skills she had already acquired and put to use.

Roxanne|Univo|Current trainee: I didn't know anything so already I learned crochet, macrame, and knitting. I have already a lot things I made with crochet like bags, scarves, potholders, blankets. So I make it [...] I look at the teacher how she do it and then I do it. I'm, I'm very, very fast learner. So I just look at it and I know it. If I don't know it I ask the teacher. Yeah. But I'm, I'm very, like, I'm very excited about how good some, some of the students they are so good. They already know how to sew. They are so good. When I look at them I say 'ohh I wish I could do that like that'. When I saw your, your clothes, I thought oh that's nice, but I can't do that yet.

In addition, being surrounded with other trainees who were more skilled sewers from the outset challenged her to rise to their level. Bandura (1997) cited that vicarious experience is learning by observing someone similar to you be successful or fail. This premise, coupled with the individual successfully accomplishing the skill increases his or her self-efficacy. For Roxanne, her success in learning how to sew contributed not only to her sense of self-efficacy but also a reconnection with her former self.

Skills training was undertaken by women of refugee backgrounds motivated to improve their lives and those of their children, family and/or community members. Many women in the training program decided to access training in a skill which they could then use to provide needed services to a "private" circle of family and friends. This was seen as a cost-saving strategy for themselves as well as family and friends. For example, Natalie a current trainee at Salas explained through a translator the cost-saving benefits of being in the training program.

Natalie|Salas|Current trainee: The benefits are that she learned how to sew her cultural dresses (dira) when she go to wedding. She said that usually she had to go to a tailor which costs money. And also we wear the underskirt too that goes with the dress, she also learn how to do [sew] it. 
She learned how to put the elastic too. Last thing is she is trying to make her dresses on her own.

Evidently through the training program Natalie developed not only a cost-saving strategy to avoiding expensive alteration costs, but also self-confidence. According to Bandura (1982) feelings of self-confidence underlie self-efficacy, which were motivated by Natalie's own personal goal of making her own garments. Expectancy (a factor in the expectancy theory equation) is shaped by self-efficacy, and every trainee had different expectations of what they felt capable of achieving (Colquitt, LePine \& Noe, 2000). Natalie and many of the trainees interviewed in this study indicated that if they kept attending their training classes it would lead to them acquiring the necessary sewing skills to either sew or repair garments from home, or become formally employed in the apparel industry.

Working from home using the sewing skills they had developed was very valuable to graduate trainees, who cited the flexibility it afforded them to spend more time with their families while they worked on bundle work or contract work; they were also able to improve their English language learning by watching television while they worked, and family members also chipped in and helped with the work. Ruth, a graduate trainee from Avenio's training program alluded to this in her interview.

Ruth|Avenio|Graduate trainee: I thought about some people who can get something to work at home. I think that's really nice. I don't [won't] have to go out and work because my mom is older so I have more time to be with her [if I am] working from home. She might can help to put small things [together].

Maintaining a strong presence in the home was also important for the mothers in Buckingham et al.,'s (2006) study. They valued skills that could more easily be plied 
from the home, where paid work could conveniently be scheduled in with childcare and other domestic arrangements were attractive.

\section{Creating a Sense of Community in a New World}

Having a collective space that was a safe, shared space that not only provided training but also cultivated solidarity and fellowship was important to trainees. Due to the circumstances associated with fleeing one's country due to war, fear of persecution and violence, many refugees had experienced interrupted education or no formal education in their home country or in the refugee camps where they lived prior to resettling in the United States (Finn, 2010). The provision of a comfortable, safe, and pressure-free learning environment was a feature that drew many non-traditional learners to join the training programs. This is important because the spaces in which training occurs can have a significant impact on trainee's response to what is being taught: either by encouraging women to take advantage of what is offered, or discouraging them from participating (Rouiller \& Goldstein, 1993; Holton, Bates, \& Ruona, 2000).

Stepping stone. For many of the trainees the program was a stepping stone from the home into the world of work, and they welcomed the flexible structure of the training programs and the support of training providers. To begin with, trainees cited practical reasons like, "it's safe," and "the timing works well." Also, the flexible characteristic of

many of the training programs' curricula accommodated the unique needs of trainees, and allowed for self-directed progress. Adult learners tend to be more self-directed than their younger counterparts (Knowles, Holton, \& Swanson, 2005). Similar findings were cited by Buckingham et al. (2006) and Raemdonck (2006), whose results indicated that self- 
directed learning gave training programs some flexibility to be tailored to the needs of the learners, and thus influenced the process of learning significantly.

Role of Training Providers. Training providers were instrumental in creating a welcoming environment for trainees as indicated by Kelly, "But after I go to a class, I think they, they the teachers actually the teachers, helped me by showing me to do stuff." Training providers played an important role in supporting the trainees during training and even after completion. Training providers not only provided informational support but also appraisal support or "encouragement" by: (a) advising trainees on how to apply the information and skills they had learned in class, and (b) showing appraisal for student progress that was a result of the training (Nijman, 2004). It was important for trainees to have a positive learning experience during the training program because a person's past educational experiences can predict the likelihood of participation in future educational experiences (OECD, 2005). So having a positive learning experience, especially as a first attempt at career transition may determine trainees' participation in future training programs. Some of the current trainees indicated that they were interested in participating in more advanced training programs upon completion of their current training.

A sense of community was further established within the training programs in the following ways: (a) solidarity and fellowship was cultivated around common interests in learning how to sew, (b) a shared past identity as refugees, and (c) sometimes even belonging to the same cultural or ethnic group was helpful. Four out five of the training programs were opened only to residents of specific residential communities. These programs were usually hosted in a familiar location on within the residential communities. So trainees in the programs not only lived within the same residential 
communities, had similar backgrounds and shared experiences of past identities as refugees, but also were within close proximity to the training spaces. Salas and Univo trainees, Justine, Roxanne, Natalie and Emma, acknowledged feeling more "comfortable" training in a familiar environment, and surrounded by women from similar circumstances versus having to negotiate a variety of learners.

Direct observations made during Salas and Univo training sessions, revealed that the majority of the current trainees in both programs were observant Muslim women, proven by their donning of the hijab. Training providers realizing that their prayer times coincided with the training sessions, had provided them prayer mats that were kept tucked away in nondescript places in the classrooms. At Salas, a Muslim trainee was observed pulling out a prayer mat, spreading it out, and then proceeding to perform her prayers -undisturbed by the activities around her. This accommodation, albeit seemingly insignificant, granted to the Muslim trainees showed a respect and sensitivity to the trainees' needs that the Somali refugees in Fangen's (2006) study in Sweden did not experience. Salas and Univo training programs wanted their trainees to keep attending their programs but did not want to undermine their religious beliefs. Therefore, they created accommodations for observant Muslim trainees' need to pray and by doing so further promoted feelings of community among program trainees, and commitment to the program. The findings of Fangen's study demonstrated how a misalignment between the expectations of a host country and refugees contributed to refugees' failure to complete a language course that ultimately impacted the ability for them to successfully integrate into Swedish society. 
In this study training programs were also regarded by some trainees as "getaways" from their home environment, and places where they could make friends and feel they belonged to a group. Natalie an elderly Somali woman revealed that she overcame feelings of isolation in her daily life by "getting out of the lonely house and coming here [to the training program and] chatting with all the young ladies and chatting with teachers and being encouraged by the teachers and just generally feeling energized and chatting with people." The need for fellowship with people of shared experiences may stem from the collectivist societies that Natalie and the rest of the trainees interviewed in this study originated from. Gambrel and Cianci (2003) point out that the most basic need of collectivist societies is the need to belong. Natalie's continued participation in the training program after two years may be partially motivated by the need to feel like she belonged to a group. Yet for other trainees like Roxanne, escape from a negative home environment was motivation enough to keep participating in the training program. Buckingham et al. (2006) cited that "getting out of the house" was the

primary motivation for all women with children under the age of five years in their study. This helped reduce feelings of isolation in the women, and improved their overall wellbeing. Illustrating that for some trainees with children, training was not undertaken to fulfil any immediate employment goals. Instead, the training course transformed the women participants by increasing their self-confidence and self-esteem which later enabled them to become more effective in other areas of their lives, one of which was finding meaningful work.

\section{Restructuring Family and Friends Relationships to Navigate a New World}


The primary role of every mentor identified through the interviews was to provide guidance and support to the trainees. Trainees were dependent on their informal network of family, friends, and training providers to assist them navigate the new world they were living in as well as the career transition process. Support was evident in the following forms: (a) spousal and family support, other times (b) through an informal peer network, and finally (c) as encouragement from community members. These different sources of support motivated a trainee to participate in a training program, and choose to later apply what they learned in practical terms once the program was completed (Kilpatrick \& Abbott-Chapman, 2002).

As stated earlier, family support was important to trainees staying motivated to participate and complete training programs. For married trainees, spousal support was pivotal to them accessing the training program. This was apparent in statements such as "either I can bus or my husband he can drop me here," "So, I am taking advantage, my husband is helping a lot," and "I have a husband after many years. [...] So currently now what I'm doing is trying to learn something and that I like actually." There were also many examples given of husbands taking care of children in the evenings so their wives could attend their training classes. Trainees also acknowledged that other family members such as siblings, and even parents that had been left in refugee camps or in their native countries were important to their success in the training programs. May, a current trainee at Univo, stated that, "Yes, I can communicate with teachers [but] if I can't explain something I call my brother and told him. My brother [speaks] perfect English. He can communicate for me." Although family members still living in their native countries could not provide financial assistance to the trainees, nonetheless they prayed 
for them and urged them to move forward with their educational opportunity so as to attain a better livelihood outcome. This was one of the instances that spirituality was discussed by trainees. Other studies (Khawaja, White, Schweitzer, \& Greenslade, 2008; Soussou, Craig, Ogren, \& Schnak, 2008; Green \& Elliot, 2010) have found that spirituality enhanced resilience and overall well-being in people of refugee backgrounds.

During the course of the training program at Univo, trainees formed an informal network among themselves, acknowledging that it was "a sisterhood community, like family," but that "nobody is obligated to do anything, but they chose to." Justine articulated the foundation on which their informal network was formed.

Justine|Univo|Current trainee: Yeah, we kind of encourage each other because we say come because if we don't come we will not have the program. So everybody's attendance affects every [program] beneficiary. So even though let's say I don't come, or I forget, or I am running late, somebody is knocking on the door and saying "Today's the sewing program." And I say "I am on the way." We kind of work together. Because to be honest, let's say you have teacher and you are teaching a class and you have 10 or more people on your list. [If] Three or four showed, you are not interested in teaching them that much. So we kinda, um, plus its money, electricity to run machine, plus I bet she's getting paid. I don't think that she is coming there for free. So why would we waste all that money, you know, for something that is to better ourselves? So we kind of bear all those things in mind and try to be there as much as we can.

Living in the same residential public housing community, with many trainees from Somalia created ideal conditions for such a network to form among Univo trainees. Trainees were cognizant that the program was for their benefit, and that although it didn't cost them anything to attend, the textile and apparel NPO that organized the program clearly was incurring some costs to keep the program running. May, another trainee at Univo also recounted how when "community members heard I was sewing, they said that it is good." Community members affirming the value of what she was doing was 
important for someone like May who is from a collectivistic culture (Eritrea), and is influenced and motivated by the opinions and support from her community to feel fulfilled (Martella \& Maass, 2000).

Mentor/mentee relationships were apparent when trainees developed relationships and sometimes even friendships with individuals who were experienced or knowledgeable in an area that they weren't. The mentors served as guides to the trainees. Mentors, many times were from the same informal network of family, friends and training providers that was discussed earlier. May explained her brother's role in giving her honest and objective advice about how to start her life in the United States if she wanted to be successful.

May|Univo|Current trainee: My brother told me you can work and you can go to school. And he want I go to school and me too I want to go to school. But I can't. I spent one month with my brother [when I arrived in the United States], and he covered the rent and I don't worry. But now his daughter and his wife come back from Ethiopia. I am alone now with a roommate. But he told me if you don't go to school and you are learning something you will have good work and make good money. But if you are not you have to be a hard-worker and work anything you can.

May's brother was resettled in the United States five years before May was, and from her interview it was evident that she completely relied on his financial support when she was still living in a refugee camp in Ethiopia. From my observations and interviews within the training programs, training providers focused on sharing their network and expertise with trainees, and generally refrained from giving out life advice. This was because training providers didn't share the same backgrounds as trainees and often felt that they were "not qualified" to give out life advice. However, training providers willingly communicated crucial information to trainees to promote positive training outcomes, such as job leads in the local apparel industry. In the case of Kelly, a trainee from 
Ethiopia, her training provider had told her "that if you are working hard and [in a] continuous way, we are going to join you to some company." Secondly, trainees reported how training providers would "assist with different tasks," "sometimes they cut and then you sew," and also teach them "about different sewing machines which I don't know." All this was done to share their knowledge and expertise with trainees and promote learning.

Ruth, a graduate trainee, from Cambodia explained how some of her training providers at Avenio offered after-hours help in the classroom to make sure they got their work completed. Training providers also found community-based resources to help graduates buy equipment to increase their chances of success in industry jobs. One of the most profound statements about the way relationships were restructured to help trainees with refugee background navigate a new world, occurred when unexpected friendships developed between training providers and trainees. Lauren and Judy, both originally from Afghanistan, were graduate trainees from Voltan's training program. Lauren explained during her interview what evolved once the training was completed.

Lauren|Voltan|Graduate trainee: I still have contact with those people and I am good friends with them. Um, especially the teacher, Ms. Emily, she's so kind and she is so humbled. We mostly see each other talk to each other and go to some places to eat or to talk. Yeah, she's, she's more like a friend and yeah, she's a nice lady.

Expanding their social network to include cultural experts will only enhance Lauren and Judy's assimilation experience into the new world that they are living in.

\section{Translating Skills and Knowledge into a new world}

Past work experience served as motivation for trainees to participate in the career transition programs and attain training outcomes because of their previous success within 
the textile and retail sectors. The majority of trainees interviews came to the career transition programs with past work experience in the textile and retail sectors in their native countries or the refugee camps where they had lived. Those with high school diplomas, had attended technical colleges in their countries of origin, and learned "various skills like sewing, and also embroidery," "buying and selling RTW [ready-towear]," and even "enjoyed teaching it [sewing classes] at refugee camps." Many had experienced success in the field and expressed that based on those "previous [experiences], you know, I come here to learn."

Having previous experience in the fashion and retail sectors was key to many trainees' interest in the career transition program. They were passionate about developing their skills to suit the U.S. apparel industry, and felt empowered when they performed well in class. Several of the trainees had on arrival in the United States sought out work in the apparel industry, but were rejected due to a lack of English language skills. Rebecca, twenty years after her first attempts to secure a job in the apparel industry, remained optimistic about reaching her goal of getting a job in the apparel industry, because "by doing this [training] maybe I can going to the factories or working from home."

\section{Physical constraints and language barriers}

Several physical constraints and language barriers served as hindrances to motivation for trainees participating in training programs and in attaining training outcomes. Physical constraints were related to: (a) the geographic location of the training program and access to it, (b) trainees' physical impediments, and (c) weather; while language barriers consisted of the lack of English language proficiency. 
Geographic Location. Training programs that were located outside of the residential communities that trainees lived posed special challenges for participants. To begin with, a lack of parking facilities required trainees either to be dropped off by family members or the use of public transportation. Natalie through her translator remarked on how this was one of her greatest challenges, "At this time it is hard for her because she's an old lady and her back is hurting and her leg is hurting. She rides the bus so it is hard for her standing in the cold and wind and waiting for the bus." Cold and wet weather negatively impacted trainees' class attendance, and one training provider equated this to the fact that some of the refugee women found it difficult to adapt to changes in their environments especially those they were unfamiliar with.

Eve|Univo|Current trainee: So the weather's bad...the weather affects a lot in, in this kind of rain, snow. Like a storm's coming. And everyone is like nowhere like, no, not going to come out. Especially Somali women they were not used to the cold. Again, this [weather] like you told me this [weather] is nothing but like for them this is cold a lot. Like I think like one of those students, she told me that this is the first time she saw the snow, I think it's Lena who was here. Last class that I met her and she'd say this is the first time they see the snow since they came. So it's, it's you never really know. But it's just little hard for them to come out then or to adopt to the new things. Like even like training program $\mathrm{X}$ is just like across from this building -that's a new building for them.

Other trainees commented on the fact that they took "two bus and the train," to class, "it is far," that "transportation to class takes a lot [of] time," and public transportation was the only option because "I don't have a car and I can't drive because of my epilepsy." Graduates like Jennifer, upon successful completion of her training program was not able to accept industry work, even after successfully interviewing for them, because of a twohour commute by public transportation that it required. 
Language Barriers. Inadequate English language skills isolated trainees from their peers and often even from their training providers, implying that there was some training information that was lost in the process. Trainees regarded the career transition programs as a preliminary step into the world of work, and acknowledged that "once you go to the high level, [you] must know English." They understood that English was the language of American society and they were expected to speak it, "otherwise [...] nobody gives you a chance to join the job.” Being unable to communicate directly with instructors, ask questions and articulate their needs, had trainees concerned about the information that they were missing out on. This directly affected their self-confidence which underlies self-efficacy. A lack of self-efficacy may eventually be reflected in a lack of skills development.

Many trainees cited their inability to attend formal ESL classes offered in the community due to scheduling conflicts, resulted in their lack English language proficiency. So finding alternative ways to learn English so they could attend the training course or be employed was commonplace among trainees. Some resorted to watching English-language television shows to improve their conversational English, others relied on their interactions with native English-speakers at their jobs to help them learn the basics, while yet others accessed free language tutorials online. What is clear is that trainees recognized the importance of English language skills to their successful integration in the United States.

\section{Childcare}

Although the women with children under the age of seven were a minority among the trainees interviewed, for those who had children under the age of seven childcare was 
significant hindrance to their motivation to participate in training and even to get a job outside the home. These women bore the full responsibility for caring of their young children. They cited a number of reasons why the availability and accessibility of safe and affordable childcare was a major problem. For low income women the cost of safe and affordable childcare may be prohibitive (Buckingham et al., 2006). Low income is not the only barrier though, Rose, a graduate trainee of Burmese origin explained how sending her son to a daycare run by a Somali woman of refugee background proved futile.

Rose|Avenio|Graduate trainee: For my son I was trying to get the best daycare to improve English skill to study about English for him. But unfortunately, um, I got um, like, a different country people like from Somalia. The daycare he was there for about nine months before he go to school. But he did he don't, they don't improve for the English skill.[...] When I was over there [at the daycare], I, I don't even know it was only Somali or another different children. For about nine months I watched him go and I never went in there [the daycare]. They [used to] pick him up and just drop him up at home. That's why I didn't know that. But um, she [the daycare provider] was on vacation about two weeks, that's the first time of the nine months I went to there, to her home and to pick him up and I see everything. Oh Wow. They're nothing teaching they just stay at home and just play. And then, and then the children there speak the same language, not English. So he [her son] said he can't talk anything. He can't understand. Yeah. He's also not happy to go to there and always like, oh, his reasons are different. But I didn't know why he said like this.

Rose felt the different cultural background of the daycare provider and the other children in her care contributed to her child's lack of English language improvement in the nine months that he attended the daycare, and was reluctant to send him back even though it was affordable care. At the center of Rose's story is the issue of trust. She trusted the daycare provider (a Somali women of refugee background) to provide her son with good care, which included teaching her son to speak English. Incidences of refugees trusting other refugees as a coping strategy in the country of resettlement was reported among 
newly resettled refugees in the United Kingdom (Morrice, 2007). Other studies have cited high levels of trust and collaboration among refugee groups living in communities as they look for work and begin to establish themselves after resettlement (Lamba, 2003; Carpenter, Daniere, \& Takahashi, 2004) So Rose's decision to trust that the daycare provider was providing her son with adequate care inspite of her son's reluctance to go, may be attributed to her lack of connections outside her community and therefore the ability to pursue alternate childcare options. However, other mothers had difficulty leaving their children with people they didn't know or trust, even if they lived in the same community. Suggesting that mistrust or restricted social networks may have contributed to low training participation rates due to unresolved childcare issues.

Incidences of education and career training disruptions due to inadequate childcare was common among these women. Justine recalls such a disruption in her education, "I was going to school [community college]. I was going to learn dental hygiene, but I couldn't find a babysitter so when I could the babysitter was too expensive for me. It was not possible because my husband worked.” Even when the training program was within the residential community, childcare still presented problems for her.

Justine|Univo|Current trainee: Because my oldest is nine and you cannot leave her like two hours, three hours with the other kids. And the youngest is...she is two years old. If sometimes I have my niece and nephews, then I leave and stay there the whole time, but when they're not there I cannot stay the full two hours. I can only spend one good hour but not two good hours. So I still have the challenge of childcare.

Here, due to U.S. child protection laws (Child Welfare Information Gateway, 2013) Justine was unable to leave her young children alone for longer than an hour at a time. So having the training program in her residential building had given Justine some access to the training program but not full access to it. Other trainees recognized how a lack of 
adequate childcare, not only hindered their motivation to participate in training but also disrupted attendance at ESL classes as well as their ability to work outside the home.

\section{Unmet Expectations}

Refugees face many challenges during resettlement, these include: (a) finding work, (b) learning the host language, and (c) accessing training programs ( $\mathrm{Li}, 2000$; Aycan \& Berry, 1996). Unmet expectations occur when refugees have unrealistic expectations about what they will experience or the level of difficulty they might experience in the new environment or within a training program (Caligiuri, Philips, Lazarova, Tarique, \& Burgi, 2001).

The unmet expectations of the trainees with refugee backgrounds interviewed with regard to the textile and apparel NPOs' training programs were the result of a lack of: (a) knowledge and understanding of the U.S. apparel industry, and (b) understanding of the goals of the training programs, despite being given the information about the programs. Trainees' past experiences with sewing classes in their native countries or in the refugee camps set the expectations for their current career transitions experiences. Their expectations of: (a) the skill-set they would gain during the training programs, (b) instructional methods that training providers would use, and (c) the resulting work opportunities that stemmed from their participation and completion of the programs, were founded on their past learning experiences. According to Norman (1999) adult learners in educational programs unlike children and adolescents make sense of new learning experiences based on their former learning experiences. This was evident in Judy's recollection of her experience as a trainee at Voltan.

Judy|Voltan|Graduate trainee: At the first, uh, it was uh, the expectation that I had, I was expecting that it would be something like sewing the 
dresses or something -like at home. But because we were the first students [in the program] and it was the first class and there was no other students, uh, there were no graduated [trainees] from those classes or training to ask.

While it was difficult to ascertain whether trainees' were misinformed or under-informed about the career transition programs they enrolled in, Judy recollection of events leading to the training program indicate that she was uninformed about the contents of the training course. However, Eve, a training provider for Univo summed up the mismatch in training program goals and trainee expectations to the organization of the apparel industries in industrialized nations versus non-industrialized nations.

Eve|Univo|Training Provider: They are used to actually fixing and uh, making their own clothes and they're country was not being um, like factorizing [industrialized] yet. So they were more close to the generations that were making and fixing their own clothes, sewing. So their appreciation for the sewing is very high. It's like they really want to learn, they really want to do it. Its valuable skills from their home country. Much of the time even in my back country, Korea or China or my Asian friends from China and India, when they were thinking about sewing, they were connecting it with a factory. Yeah. Yeah. [...] My mom she never really make clothes for me. So I'm like, we kind of are the generation that are buying. We are used to buying our clothes not custom-making clothes or altered clothes or something like that.

This further confirmed the fact that trainees' expectations were grounded in experiences in their past. In many developing countries the apparel industry has not reached a point where mass production of clothing is commonplace. With low labor costs, neighborhood tailors and seamstresses are still able to meet the clothing needs and wants of the common person, and earn a living. Ready-to-wear garments that the masses can afford are in the form of second-hand goods. In developed countries, such as the United States this is not the case, the apparel industry is very structured and heavily regulated, with the majority of goods being mass-produced in factories. 
Finally, regular skills assessment and feedback was desired by trainees, for them to determine their progress in the program. A skills assessment was carried out at the start and conclusion of most training programs, but not periodically through the duration of the training. For trainees, feedback was important and necessary in order for them to correct mistakes and be held accountable for their progress.

\section{Learned Dependency}

Protracted refugee situations often result in refugee camp conditions that foster helplessness and reliance on international aid for refugees to survive (Abdi, 2005). In situations where refugees lived in closed camps, there was little opportunity for them to find employment or make a living. De Vriese (2006) noted when examining refugees' livelihood strategies in camps that refugees were unlikely to reveal the efforts they took to sustain themselves, for fear that they would not remain eligible for continued humanitarian assistance. De Vriese (2006) concluded that this showed evidence of a dependency that had developed among the refugees for humanitarian aid.

May|Univo|Current trainee: Yeah, I am living in Ethiopia as a refugee and sometimes they help me-UNITA in Ethiopia. One of my brothers came here [United States] and he support me a lot from the United States. In Ethiopia it is good but you can't make money as a refugee. Because you don't have any license to work. So it's difficult [to get] what you want. You want to do something but you can't that much."

In May's statement she reiterated the importance of remittances to refugees like herself who were living in refugee camps and unable to work. The inability to work or earn a livelihood due to national refugee laws and policies, and the dependence on humanitarian assistance to provide all of one's needs because there are no other alternatives, resulted in total dependence on aid (Abdi, 2005). Some researchers have ascribed this behavior to be a reflection on the humanitarian agencies rather than refugees 
(Kaiser, 2001; Bakewell, 2003). De Vriese (2006) stated that when refugees living in camps have opportunities to get benefits, they "will present themselves as needy and will try and get what they can (p. 13)." If benefits are based on need then individuals will present themselves as needy to access the benefits (De Vriese, 2006). Some of that learned behavior translated into current trainees' motivation to participate in training not relating to getting a job in the apparel industry, but keeping their welfare benefits. When asked about the support she received from her community, Justine acknowledged that "the government helps us pay some of the rent," for the family's apartment. While another trainee, Kelly, disclosed that her family was "on the waiting list" to get into government subsidized housing. Erin, was not impressed with some of her fellow trainees in the career training programs who she felt were taking advantage of the system.

Erin|Leventis|Graduate trainee: I tell Rayna that they should take away all their [social] benefits, if they want to see them go to work. They will have better attendance in class too. They only come to class to keep their benefits. They sometimes tell me I am stupid to work, because why would I make $\$ 10$ to spend $\$ 9$ on benefits that are free? They tell me I am stupid to work.

Erin's evaluation of the reasons why many refugees were inconsistent in attending Leventis' training program was echoed by Rayna during her interview. Suggesting that the "dependency syndrome" that De Vriese (2006) had identified while studying refugee livelihood strategies in camps, had manifested itself among the trainee population at Avenio too.

Justine, a trainee, mentioned during her interview that she was "fighting for a homework helper" for the kids in her residential building. The current homework tutor was located in a community center less than 10 minutes away by foot. Her reasoning for "fighting" for a homework tutor for her building was that "the kids had to cross a busy 
street to get to the community center" and that meant that parents would have to accompany their children. This expectation that programs and services should be at a convenient location to the targeted refugee population was one of the reasons Univo moved its training program to public housing residential building close to the population it was targeting.

\section{Conceptual Model}

There were 9 emergent themes in this study: (a) informal pathways of training programs, (b) building foundations for a new life, (c) creating a sense of community, (d) restructuring family and friend relationships to navigate a new world, (e) translating skills and knowledge into a new world, (f) physical constraints and language barriers, (g) childcare, (e) unmet expectations, and (f) learned dependency.

Resiliency, was the ultimate outcome for trainees in this study, and represented the coping strengths that trainees demonstrated in overcoming obstacles that hindered their motivation to participate in the training programs and attain training outcomes. These themes were interpreted in a holistic manner throughout the data analysis stage. A visual representation of the conditions that facilitated or hindered the trainees' motivation to participate in career transitions was created, based on researcher's personal reflection, and interaction with the participants in the study (see Figure 5). 


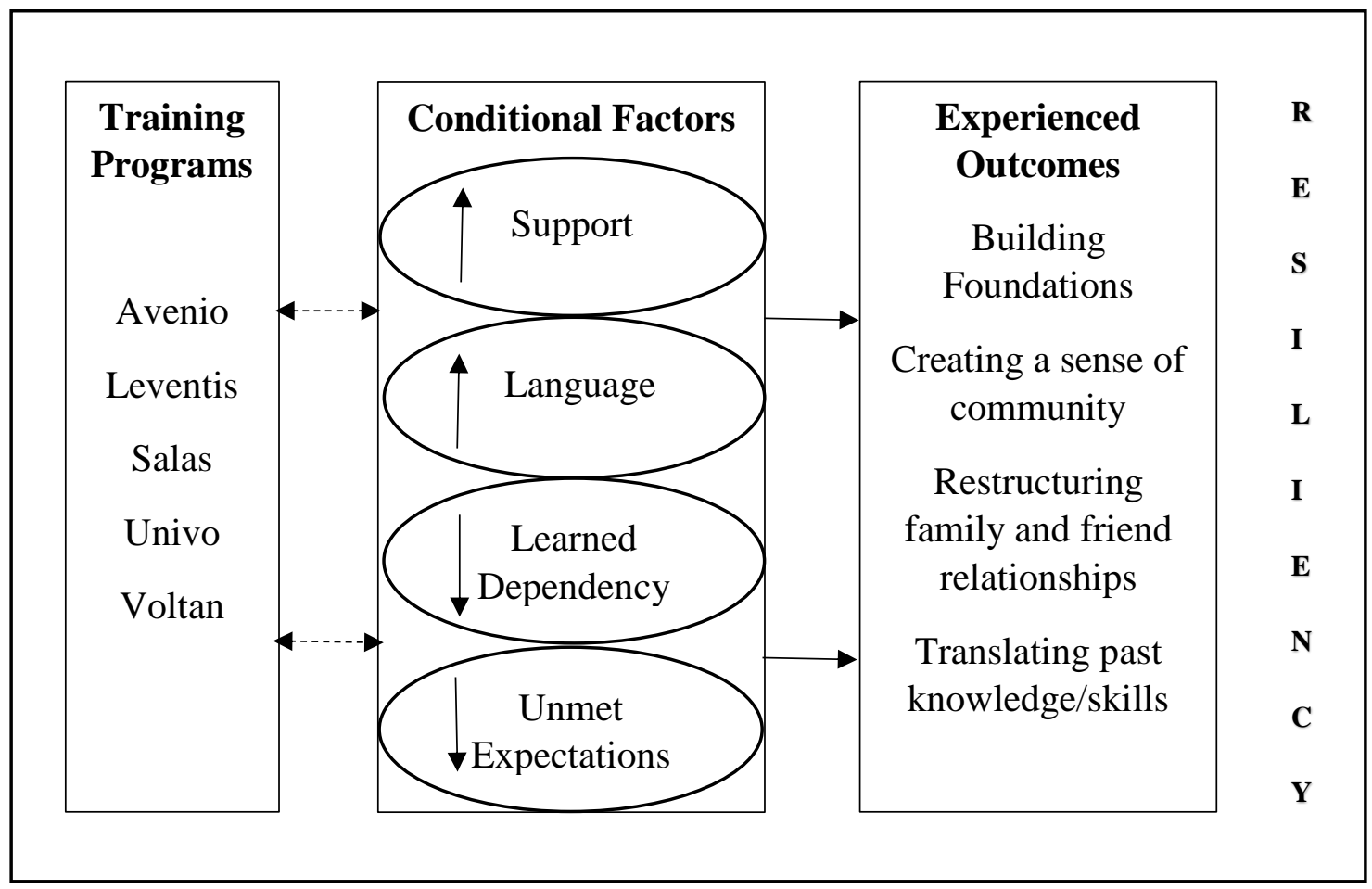

Figure 5. A visual representation of how conditional factors in trainees' lives facilitated training motivation and outcomes.

\section{Expectancy Theory and Motivation of Trainees}

In this study expectancy theory of motivation is used to explain the task-related effort required by a trainee to participate in training programs and attain training outcomes. Vroom (1964) identified three criteria that needed to be met if a person was to be motivated. To begin with, a person must expect that he or she is able to perform the specific behavior that is instrumental to achieving a specific outcome or reward (expectancy). In this study, expectancy is based on past experience, self-efficacy, and the perceived difficulty of the goal. Second, a person must believe that a specific behavior is instrumental to achieving the specific outcome or reward (instrumentality). Lastly, a person must value attaining a specific outcome or reward over not attaining it. Valence is the value a person attaches to a reward or outcome. 
What this process model represents is how trainees with refugee backgrounds were motivated to participate in training programs, and what outcomes they experienced as a result. The model depicts all 9 themes that emerged as a result of data analysis. Figure 5 represents the holistic process of the career transition experiences of women trainees with refugee backgrounds in textile and apparel NPOs. The process starts off with the five training programs that represent the places in which career transitions occurred for the trainees in this study. Two-way, dashed arrows between the first and second portions of the process model illustrate the informal pathways of training programs; trainees had the ability to move between programs as long as they met program enrollment requirements (e.g. resided in public housing, had a refugee background, or had certain sewing skill set) even though most of them remained in the programs they initially selected. The ability to move between the career training programs allowed trainees to participate in programs that met their needs and thereby increased training motivation. For example, some training providers, recognizing the unique challenges facing their trainees, were instrumental in designing programs and providing services that helped trainees overcome obstacles that would hinder their motivation to participate. Most of the training programs addressed the lack of English language skills among trainees, by incorporating step-by-step physical demonstrations of every project assigned, and sometimes also provided trainees a hand-out with visuals. Many graduate trainees found step-by-step demonstrations and visual hand-outs helpful, because not only did they lack English language proficiency at the time, but were preliterate when they were enrolled in the skills training program. Voltan did something different. This textile and apparel NPO addressed the lack of English language skills 
typical to newly resettled refugees by limiting trainees enrolled in their sewing programs to a small group of eight Afghani women. Haley, Voltan's training provider explained why this was the case.

Haley|Voltan|Training provider: We have volunteers who um, in each class there's up to four volunteers, you're making sure everybody's staying on track. If someone's machine gets jammed or needs to change a needle then they are there. They're able to stay together and then you're keeping everybody kind of on track as the project goes on.

Volunteers came from a local church as well as the textile and apparel NPO itself. Voltan's program matched one volunteer to every two students to ensure that training goals were met. There was much less opportunity for self-directed learning in this training program. Voltan also used flash cards to help trainees associate specific words with sewing-related items.

Second, four conditional factors that facilitated the trainees' motivation to participate in career training programs and attain training outcomes are presented. The factors are: (a) increased support, (b) increased language skills, (c) decreased learned dependency, and (d) decreased unmet expectations. When trainees experienced increased support from training providers, family and friends in the form of favorable scheduling, childcare assistance, familiar location of programs, etc., they were motivated to participate in the training programs and as they continued with training they experienced outcomes that built their soft skills. The same can be said for trainees who experienced increased English language comprehension when training providers produced learning aids to help them understand what was being taught. Decreased levels of learned dependency occurred when trainees were motivated to become more economically selfsufficient by participating in career training programs and learning a skill. They too were 
motivated to keep participating in the training as they experienced some of the outcomes that resulted. Lastly, when trainees were able to adjust (lower) their expectations either of the type of instruction they would get, or the work they would qualify for as a result of the training, they experienced less dissatisfaction with their training experiences and their motivation wasn't diminished. This was evident when trainees were faced with inadequate numbers of sewing machines and training providers to accommodate all their needs in the program, trainees remained patient and still kept attending the training with the assurance that the problems would be resolved. There was a sense of optimism among the trainees even when the textile and apparel NPOs had fallen short of meeting all their expectations. This adjustment enabled trainees to continue participating in the career training and experience outcomes that didn't immediately translate into dollars and cents. These coping strategies used by trainees to overcome obstacles to training motivation, are evidence of their resiliency.

Lastly, with these four conditional factors present, trainees were able to experience the following training outcomes: (a) building foundations for a new life, (b) creating a sense of community, (c) restructuring family, friends, and community relationships to navigate a new world, and (d) translating skills and knowledge into a new world.

In conclusion, motivation was responsible for trainees' directed actions towards a specific behavior (Vroom, 1964). Their desire to participate in training was initiated by textile and apparel NPOs providing programming that addressed their needs, motivations, or past experiences. The four conditional factors that were present in a trainee's career transition experience boosted their confidence in the belief that their training participation 
would lead to satisfactory outcomes. This four factors facilitated trainees' motivation to participate in the training. The outcomes experienced by trainees' in career transitions were of value to them, and explained why trainees preferred attaining these outcomes versus not attaining them at all. The realization of these outcomes helped sustain trainees' motivation to keep participating in the training programs.

\section{Researcher's reflection}

When interviewing and interacting with training providers in each of the five textile and apparel NPOs, their strong commitment to uplifting the socioeconomic conditions of their refugee trainees was evident. I discovered that these five textile and apparel NPOs had formed an informal network as a matter of survival. The network functioned as a referral system for potential students, directing new trainees to training programs that matched the residential requirements, recommending others to more advanced training programs once a basic skill-set had been developed, and sharing curriculum. All the programs were funded by grants, and so much of this was done to achieve the enrollment numbers required by their donors to keep the programs running. With programs running on grant money, many of the owners of the textile and apparel NPOs, their board members, and instructors were in unpaid positions. This restricted the availability of instructors to trainees, and discouraged instructors from following through with trainees once they had completed the program.

Socioeconomically, I felt at par with the trainees, and during the training sessions I attended, I stressed that I was a African student doing a research project as part of my university degree work. I didn't emphasize that I was a researcher nor mention that I was working on a doctorate. Most of the participants that I interacted with were dressed 
casually, or in hijab with long loose-fitting dresses that had long flowing sleeves. I dressed casually too as a student would, donning jeans, a sweatshirt and tennis shoes. I used my school back-pack to carry my research materials and recording devices to each data collection site.

Between direct and participant observations, I didn't have much time to build rapport with each potential participant. The initial presentation I gave on African textiles and my dissertation research, at the beginning of each training session I observed, was met with lukewarm reception. Thus, I had to work on building rapport with potential participants during the direct and participant observations I was making. When it came to recruiting potential participants, I ended up relying on the training providers to 'close the sale' for me. There could have been some bias on the part of the training providers, in which trainees they chose to assist me with recruiting for my study. However, I believe that I got an accurate picture of the career transition experiences that trainees had in the five organizations I selected. Looking back, I should have brought light refreshments to the training sessions as some of them lasted three hours, and then if interviews were conducted at the end of the sessions, that further extended the time research participants had to spend at the training center. I should have included a small monetary incentive for those trainees that participated in the study, this incentive may have helped recruiting efforts yield results earlier than they did.

Most of the interviews I conducted with training providers were in person, and I found them not only interested in the project but grateful for the opportunity to discuss a topic that was important to them. They openly shared their experiences and perceptions about the training programs and their trainees, gave me current information about their 
NPO, as well as a tour of their facilities. Scheduling interviews with the trainees proved to be challenging, because: (a) some trainees gave me incorrect phone numbers, (b) a few trainees had scheduling conflicts due to previous engagements or childcare issues that could not be resolved easily, and (c) yet other trainees chose to schedule their interviews when I was at the airport flying back home, or at a date when I was already back home. In hindsight, I should have planned for a longer stay (three to four weeks) to complete my data collection, and worked to conduct the majority of interviews in person. However, I made sure that all interviews were conducted within a two-week period from first contact.

To conduct interviews over the phone with trainees, I had to begin by building rapport and that was done by remaining upbeat, maintaining a friendly tone, being interested in the narrative, and expressing empathy when necessary. I felt that the participants freely and honestly discussed their career transition experiences during their interviews. The two most difficult parts of the interviews with trainees centered on content and accents. In terms of content, some of the trainees' narratives were difficult to listen to because they were steeped in trauma, neglect and hardships. However, I allowed the women to freely tell their stories and offered empathy when I could. There were a couple of instances where the narratives took such a mental toll on me that I had to ask the interviewee to reschedule the rest of the interview the following day. If these difficult interviews had been conducted face-to-face, then I wouldn't have had a means of escape from difficult conversations. Also, had the interviews with trainees been conducted within the premises of the training facility, I believe the interviewees may not have been as open and forthcoming about the reality of their training experiences as they were. 
Trainees in this study came from different countries, and most of them still spoke English with their native accents. At first they were difficult to understand, but I found myself developing coping strategies such as 'mirroring' what was said, in order to improve my comprehension. Mirroring is a technique often used during therapy, where the therapist repeats back to the client, word for word, what the client just expressed (Schreiner, 2014). I also found myself often asking interviewees to give me examples of their statements, as a way to ensure that their responses were as clear of ambiguity as was possible. Had I conducted interviews in person, I don't believe it would have made much of a difference, since the transcription of all interviews were done directly from a digital recording of the conversations. Transcribing these interviews took a lot time, as I often listened to interviews four or five times to ascertain what was being said. Next time, I work with a refugee population I need to build more time into the interview transcription process to allow for understanding of interviewees' accents.

During the week of data collection I relied solely on the city's public transportation to take me from one location to another. I wanted the experience of relying on public transportation to take me to the various training programs. I found the public transportation system of light rail and buses to be reliable, efficient, and relatively cheap. To access the right bus or train, I was completely dependent on my Google maps app on my smartphone. I could plan my trips at least three hours in advance, and was able to see light rail and bus schedules as well as recommended walking routes to each stop and from each stop to my final destination. I also found bus drivers were helpful with transfers. Although temperatures were mild, it rained all day, every day of that week. 
Reflecting on the research project, I found that setting up, conducting, analyzing, and interpreting a multi-case study has helped strengthen my skills as a qualitative researcher. To begin with, I now understand how important it is to have a gatekeeper who will support your inquiry from start to finish. Rayna, my personal contact, was that gatekeeper. She provided the necessary access to the textile and apparel NPO training programs and also to the trainees, indirectly. I have also learned that even if you have a case study protocol, with qualitative research, it is important to be patient and remain somewhat flexible throughout the research process, allowing the study's natural design to surface. Conducting this project confirmed the importance of using data triangulation and methodological triangulation in qualitative inquiry, in order to more accurately explain the intricacies of human behavior. Lastly, organization was key. Keeping accurate field notes, observational protocols, and transcribed interviews was important to establish trustworthiness.

Although, this group of participants (i.e. the trainees of refugee background) were considered a vulnerable population and therefore hard-to-access, I believe the discoveries that were made as a result of having them participate in this study are well worth it.

\section{Summary}

In this study, after data collection all 24 interviews with participants were transcribed by the researcher. Qualitative thematic analysis was used to explore the interview data. Any field observations that were recorded in the researcher's field notebook, observation protocol, and artifacts and documents were used to corroborate the interview data. Nine themes emerged from the thematic analysis of the interview data: (a) informal pathways of training programs, (b) building foundations for a new life, (c) 
creating a sense of community, (d) restructuring family and friend relationships to navigate a new world, (e) translating skills and knowledge into a new world, (f) physical constraints and language barriers, (g) childcare, (h) unmet expectations, and (i) learned dependency. People of refugee backgrounds experience many challenges during the resettlement process (Fangen, 2006). However, developing strategies to overcome obstacles that would otherwise prevent their participation in training programs and attainment of training outcomes, was pervasive among the trainees in this study. It was evident that the coping strategies that trainees developed made them more resilient.

Lastly, a conceptual model was developed as a visual representation of these nine major themes that emerged from the study. It was made up of a three part process that depicted the training programs, conditional factors and experienced outcomes, of the trainees in the study. Resiliency was the ultimate outcome experienced by trainees. 


\section{CHAPTER V: DISCUSSION AND CONCLUSION}

Chapter V contains the following sections: (a) overview of the study, (b)

contributions and implications to theory and practice, and (c) study limitations and future research opportunities.

\section{Overview of the Study}

Career transitions play an important role in the development of work-appropriate skills. Several empirical studies have pointed to how individual characteristics (Ree, Carretta \& Teachout, 1995; Warr \& Bruce, 1995; Colquitt, LePine \& Noe, 2000), motivation to participate in trainings (Tannenbaum \& Yukl, 1992; Colquitt, LePine \& Noe, 2000), and instructional methods for training (Salas \& Cannon-bowers, 2000; Taylor \& Giannantonio, 1990; Knowles, 1990; Plimmer \& Schmidt, 2007), impact the effectiveness of training. The goal of the U.S. refugee resettlement program is for refugees resettled in the United States to become self-sufficient within 18 months of arrival on U.S. soil (U.S. Department of State, 2017). So for refugees, skills training or re-training in areas that match the needs of the local labor market is essential to their economic integration, once resettled. With the re-birth of manufacturing in the northwest region of the U.S., local textile and apparel NPOs set-up community-based sewing programs that provided skills-building and job training opportunities to refugees resettled in the region (Workforce Training and Education Coordinating Board, 2008). However, training providers reported high rates of attrition from their programs, with trainees of refugee background either failing to complete training courses or completing training courses but not transitioning to full-time jobs in the apparel industry (Rayna, personal communication, November 4, 2016; Claire, personal communication, November 9, 2016). Literature in the career training, refugee resettlement, and clothing and textiles 
disciplines has not addressed what motivates women of refugee background to participate in career transitions and/or attain training outcomes nor what the role of U.S. textile and apparel NPOs in the vocational rehabilitation of refugees resettled in the United States. Therefore, the aim of this study was to gain in-depth understanding of the career transition experiences of women with refugee backgrounds within the context of textile and apparel NPOs in the United States by exploring what motivated them to participate in the training programs and attain training outcomes. To that end, this study proposed the following research questions:

Research question 1: What facilitates the motivation of women trainees of refugee backgrounds to participate in career transition programs and attain training outcomes?

Research question 2: What hinders the motivation of women trainees of refugee backgrounds to participate in career transition programs and not attain training outcomes?

Answers to these two research questions were found through participant observations, direct observations and in-depth interviews that were conducted at five textile and apparel NPO training sites. Using a multiple case study approach, 14 graduate and current trainees as well as 10 training providers in each textile and apparel NPO who were knowledgeable about the organization's structure, vision and mission were interviewed. The data analysis uncovered nine emergent themes that represented factors that facilitated or hindered the motivation of trainees to participate in career transition programs and attain training outcomes. These findings are discussed next.

To begin with, the data revealed that multiple informal pathways to the training programs existed for trainees to attain the sewing skills training they sought after. 
Trainees had three different informal pathways to training programs that led to different training outcomes. As long as trainees met each program's enrollment requirements, they were not restricted to any one program or pathway, and thus experienced one of two training outcomes: (a) finding a job in the apparel industry, or (b) sewing for personal fulfillment. This flexibility, is a characteristic of many non-accredited training programs, and is more suited to non-traditional adult learners (Buckingham et al., 2006). Therefore, this characteristic may have facilitated the motivation of trainees to participate in career transition programs and attain training outcomes.

Two, building foundations for a new life for the trainee meant trainees began the process by finding the training programs that addressed their needs. Their needs centered on: (a) the training program being close to their homes, (b) if they had young children, then they had to resolve childcare issues, and (c) the training environment had to feel safe and welcoming. Trainees were motivated to undergo skills training to improve their lives, those of their families, friends and community members. Graduate trainees valued the flexibility their new skills afforded them. They could spend more time at home with their families, as they worked on bundle work or contract work.

Three, a sense of community among trainees was cultivated by a learning environment that was safe and stress-free, made up of women with common interests, and who shared a refugee background and sometimes were of the same culture. Given the trainees' refugee backgrounds, many had experienced episodes of interrupted education or had no formal education at all. The training programs were the first steps into the world of work in the United States for many trainees. So support from training providers through the application of a flexible training curriculum that encourage self-directed 
learning, was instrumental to creating this sense of community. In programs that supported self-directed learning, the individual trainee decided and managed what learning activities they undertook, and when and how quickly they completed them.

Four, relationships with family and friends underwent a restructuring so that trainees could participate in training. Spouses of trainees assisted with providing transportation to the training if the training was at a location that was far from home, and even assumed extra childcare responsibilities. Some trainees also formed an informal peer network among themselves to motivate each other to keep up with the training. Community members affirming the value of their training behavior to the trainees encouraged them to keep participating. Trainees also developed mentor/mentee relationships with their training providers who were more knowledgeable in the area of work in the U.S. apparel industry than they were.

Five, translating skills and knowledge into a new world entailed trainees using past experiences in textiles and apparel sectors as a foundation on which to develop skills that were more suitable to the U.S. apparel industry. Having some skills and knowledge about apparel and textiles helped not only initiate motivation in trainees but also directed it. Those trainees who didn't have this background were from cultural backgrounds where sewing was considered an appropriate and desirable skill for women to have.

Six, geographic location and access to training programs, inclement weather, and physical impediments, served as physical constraints to motivation. Lack of English language proficiency created a language barrier during training. Trainees that had to take public transportation in order to access the program were particularly challenged by the amount of time they spent on their commute, and the number of transfers that they made 
to get to their destination. Inclement weather (e.g. cold temperatures, rain, ice, and snow) reduced the motivation to participate in training. A lack of English language proficiency isolated trainees from some of their peers, training providers, and reduced their overall sense of self-confidence. This may have negatively affected their training outcomes.

Seven, for trainees who had children under the age of seven years, inadequate childcare was a significant hindrance to training motivation. Finding safe, affordable, and adequate childcare proved difficult for some, especially for the newly resettled refugee women. These new arrivals had a limited social network, and so their training experiences were disrupted.

Eight, trainees, due to a lack of adequate information and general understanding of the U.S. apparel industry trainees had unrealistic expectations of the training program outcomes. Their expectations stemmed from past experiences with sewing programs in their native countries or in the refugee camps where they had lived. This mismatch in training goals and trainee expectations was as a hindrance to training motivation, as dissatisfaction was experienced by trainees.

Nine, many trainees spent many years in refugee camps, unable to work and earn a living to support themselves. They had to rely on international aid and remittances from family members to survive. Now that they were resettled in the United States, the tendency to remain dependent on the government safety net was evident. Some trainees attended training for the sake of fulfilling the requirements needed to keep their welfare benefits, not to find work. This resulted in 'token participation.' 


\section{Contributions and Implications}

This study resulted in several important contributions to career training and refugee resettlement literatures, especially in relation to the U.S. apparel industry. What follows is a discussion of the study's contributions and implications for theory development, industry, training, and policy.

\section{Contributions and Implications for Theory}

This study applied the expectancy theory of motivation to explain the factors that facilitated or hindered trainees' motivation to participate in training programs and attain training outcomes. Findings addressed how their participation in training programs was initiated, directed and sustained by motivation. These findings contributed to the understanding of the intricacies of training motivation in refugee women.

While expectancy theory of motivation has been utilized in many studies pertaining to training behavior in other fields, during the literature search, none were found that specifically applied to the textiles and apparel discipline or to refugee resettlement studies. Therefore, this study's major contribution to theory is that it supported the applicability of the expectancy theory of motivation to both the textile and apparel discipline and the refugee resettlement studies. The expectancy theory of motivation was applied to training contexts in textile and apparel NPOs. Motivation was found to be pivotal in initiating, directing, and sustaining the training behavior of training program participants. The participants who underwent training in these programs were women of refugee backgrounds. These women were motivated to participate in the training programs offered by textile and apparel NPOs because they believed that they could perform the tasks assigned (expectancy), acquire the necessary sewing skills 
(instrumentality), and their new skills would lead to work in the apparel industry (valence). This outcome would satisfy a need they had.

Another contribution to the expectancy theory of motivation, was the indication that certain conditions, if present in the women of refugee backgrounds' training experiences, positively influenced training motivation. If there were increased levels of support from family, friends, and/or community members, as well as increased levels of language comprehension, then trainees were more motivated to participate in training. If trainees sought to become less reliant on the social safety net and more economically self-sufficient then they too remained motivated to continue on with their training program. Finally, as long as trainees managed their training expectations and experienced less dissatisfaction with training outcomes, their motivation to participate in training was not hindered.

Next, trainees in this study were motivated to participate in the training programs by a reward or outcome that they desired even before training began. This confirms the third assumption of the expectancy theory of motivation -people want different things from an organization or program (Vroom, 1964). However, when training program goals didn't match their desired outcome, trainees still participated in the training programs. Suggesting that they either saw value in some of the experienced outcomes or that they had adjusted what their own desired outcome was because they found value in what they were experiencing. Therefore supporting the fourth assumption of the expectancy theory of motivation -people will make choices that maximize their personal outcomes (Vroom, 1964). 
Finally, this study also made a methodological contribution to theory. Much of the research utilizing the expectancy theory of motivation is based on quantitative approaches to inquiry. Vroom (1964) developed the expectancy theory of motivation to not only explain but to predict the task-related effort used by a person. The three factors that make up the motivation equation (Expectancy $\mathrm{x}$ Instrumentality $\mathrm{x}$ Valence) all have assigned values that range from -1 to 1 , so that their given levels are easily measured. This makes research studies that use quantitative methods, a natural fit. This study prescribed to the qualitative paradigm, and adopted the constructivist approach that multiple realities exist that are shaped by context. Thus, qualitative methods to data collection were applied to the study that supported the ontological and epistemological nature of the inquiry. The determination of whether training motivation existed among participants was made through the interpretation of textual data versus numerical data. The findings from the study indicated that the expectancy theory of motivation can be successfully applied to qualitative inquiries too.

\section{Contributions and Implications for Practice}

This study made several contributions to the existing body of literature. First, one of the tenets of U.S. refugee resettlement policy is to get newly resettled refugees selfsufficient as quickly as possible. Based on the backgrounds and circumstances that the women of refugee backgrounds in this study originated from, this goal was problematic. This supported findings from other studies on refugee resettlement, which site a myriad of problems related to this policy (Fangen, 2006; Tshabangu-Soko \& Caron, 2011; Sienkiewicz et al., 2013). It was nearly impossible for refugees to become self-sufficient within 18 months of their arrival to the United States, without a firm command of the 
English language (Fangen, 2006). Many of the women of refugee backgrounds who were interviewed for this study, spoke at least two languages when they arrived in the United States. So, language acquisition does not appear to be a problem for them, rather, they cited the lack of time and support afforded to them from the resettlement agencies when they are newly arrived, to learn the English language. Without the proper English language skills, refugees claimed to have missed important opportunities (e.g. work) and information (e.g. knowledge imparted during training) that could have helped with integration. Refugee resettlement policy may consider a provision that gives newly resettled refugees in the United States a two year window, whereby they are supported (subsidized housing, childcare, eldercare, etc.) and given the opportunity to learn the English language.

Second, the women trainees of refugee backgrounds highlighted what Fangen (2006) and Colquitt et al., (2000) had cited concerning educational levels of resettled refugees who had lived in refugee camps. On average the women of refugee backgrounds in this study reported spending eight to ten years living in refugee camps, and many as minors in their formative years. Elementary and secondary education attendance was negligible. For those trainees who were adults during their time in the camps, employment was hard to find and they relied on rations from the humanitarian organizations. The Refugee Agency (UNHCR) in their humanitarian efforts towards refugees may need to further consider formal education and employment for refugees while they are in the camps. Having educational and employment opportunities may help refugees develop realistic expectations about life and work that could be transferred to their countries of resettlement, thereby assisting with integration. Also, providing formal 
educational and employment opportunities to refugees may alleviate issues of learned dependency.

Third, the information garnered in this study about the activities of U.S. textile and apparel NPOs confirmed that due to limited resources and overwhelming demand, resettlement agencies can only offer newly resettled refugees limited support in the areas of employment and career/vocational counseling and continuing education that develops relevant vocational and language skills (Keiler, 2017; Bowen, 2016; Neilsen, 2015). As a result of this ongoing unmet need, textile and apparel NPOs have stepped in to assist with settlement and integration efforts. The non-accredited community-based training programs explored in this study were used by women of refugee backgrounds as stepping stones from the home to the world of work. While participants appreciated the selfdirected aspect of many of the programs, some structure was preferred especially in terms of a clear curriculum with learning objectives. Participants also cited that these programs needed to factor in time within the sessions whereby trainees could work on and get help from instructors on personal projects.

Fourth, none of the training providers in the textile and apparel NPOs leveraged their alumnae network in their recruiting and retention efforts. This was not a strategy used in any of vocational training programs cited in the clothing and textile literature or the refugee resettlement literature, but is one that is employed in the traditional educational sector (Ivy, 2008). Several of the graduate trainees interviewed in this study expressed the desire to stay connected to the training programs that they had completed, and give back to them in some fashion. This would be an excellent way for both sets of trainees (current and graduate) to grow their limited social and work networks by 
developing mentor/mentee relationships. It may encourage more trainees to complete the training and transition to work in the apparel industry.

Fifth, Zunker (1990) found that refugees were unable to access educational opportunities due to family obligations. Many of the trainees in this study also indicated that they were unable to access ESL classes in the community due to family or work obligations. If more textile and apparel NPOs incorporated English language learning into their training program curriculum, this may be a benefit to trainees. Especially those who live with families or in communities where their native language use is prevalent, and English isn't spoken enough to promote proficiency.

Sixth, refugee women face barriers to entering the labor market that are related to language, gender and family obligations, and employer attitudes (van Kooy, 2016), and for some self-employment or entrepreneurship was an attractive alternative. Trainees in this study who weren't motivated to participate in training because of a future job in the apparel industry, wanted to use their new skills to develop a "home business." However, they typically lacked the equipment, materials and know-how to do so. Textile and apparel NPOs may consider incorporating some information about how to set up a small business, to inform those trainees that see themselves as future entrepreneurs.

Lastly, four out of five of the textile and apparel NPOs that participated in this study had learning outcomes that promoted the tenet of U.S. refugee resettlement policy that states that it is important to get newly resettled refugees self-sufficient as quickly as possible. They did this by promoting training outcomes that were related to finding work. However, the findings from this study indicated that the training programs offered by the textile and apparel NPOs are successful, but in building soft skills that will help their 
trainee's future work efforts. These skills are also important in promoting self-sufficiency in refugees, as they promote resiliency (Toth, 2003). Textile and apparel NPOs need to consider these outcomes, and realign their current goals to match them.

\section{Limitations and Recommendations for Future Study}

Several limitations were identified in this study, therefore opening up opportunities for future research. To begin with, the researcher used purposive sampling to target participants, all of whom were either current or graduate trainees, or training providers in textile and apparel NPO sewing skills training programs, located in a particular geographic area. This restriction in sampling constrained generalizability to training programs and training experiences of women trainees of refugee backgrounds in one geographic area in the United States. Repeating the study in other geographic areas with similar sampling criteria is recommended to ascertain whether trainees with different refugee backgrounds and resettlement experiences will produce different findings. In addition, repeating the study to include trainees that dropped out of the program may yield even more evidence as to the reasons why some women trainees with refugee backgrounds lack the appropriate coping strategies. A couple of the textile and apparel NPOs enrolled men with refugee backgrounds into their training programs. Expanding the scope of this study to explore the career transition experiences of male trainees, will lead to a comparison of how their motivation to participate in training differs from that of their female counterparts.

Second, the research participants represented women of refugee backgrounds living in a variety of low-income public housing in Seattle WA. Although many face-to face interviews were scheduled and conducted with participants during the researcher's 
weeklong visit to the five data collection sites, scheduling conflicts made it necessary to conduct some of the interviews by phone. This may have affected the depth of information that was garnered from each phone interview.

Third, the length of time spent in the training program by trainees and training providers was not determined during the interview process. Length of time with the training program is an important factor and can influence a trainees' overall experience and outlook on the training program as changes in staff and curriculum occur. It is also important to ascertain how long ago it was that program graduates left the program, as time affects the ability to recall activities clearly. The same would be applicable to training providers, whose tenure with a particular training program could be indicative of their commitment to program outcomes. A future study on career transitions should ascertain the length of time participants had spent in the program whether as a trainee or training provider for the aforementioned reasons.

Fourth, the study did not interview graduate and current trainees from the same textile and apparel NPO. Graduate trainees were obtained from only two textile and apparel NPOs; having the current trainees from the same textile and apparel NPO would have contributed to a better understanding of their overall training experiences. The same can be said of the current trainees that were program participants in the remaining three textile and apparel NPOs. Further research is recommended to develop a more nuanced exploration of each of the five NPOs, understanding that the findings will be enriched and applicable to each NPO.

Lastly, the theoretical framework used for this study was not a natural fit for this inquiry. While expectancy theory of motivation supposes the involvement of cognitive 
processes that are not visible, contemporary theories of motivation acknowledge that motivation is not a singular phenomenon, but rather that it consists of interactions between a person and a larger social context. I would recommend a replication of this study with the use of a more contemporary motivational process theory such as the expectancy-value theory. With the use of expectancy-value theory as the theoretical lens, factors have been added for both intrinsic and extrinsic motivations and other variables such as personal abilities and skills thereby a potentially more nuanced conceptual framework might emerge providing greater contributions to theory, government, and industry. 


\section{References}

Abdi, A. M. (2005). In Limbo: Dependency, insecurity, and identity amongst Somali Refugees in Dadaab camps. Refuge: Canada's Journal on Refugees, 22(2), 6-14.

Adler, S. (1977). Maslow's need hierarchy and the adjustment of immigrants. International Migration Review, 444-451.

Ajzen, I. (1985). From intentions to actions: A theory of planned behavior. In J. Kuhl \& J. Beckman (Eds.), Action control: From cognition to behavior. New York:Springer-Verlag.

Ajzen, I., \& Madden, T.J. (1986). Prediction of goal-directed behavior: Attitudes,intentions, and perceived behavioral control. Journal of Experimental Social Psychology, 22, 453-474.

Akresh, I. R., Massey, D. S., \& Frank, R. (2014). Beyond English Proficiency: Rethinking Immigrant Integration. Social Science Research, 45, 200-210.

Alatis, J. E. (1979). The Role of Esl in Bilingual Education. NABE Journal, 3(3), 27-37.

Alderfer, C. P. (1972). Existence, relatedness, and growth: Human needs in organizational settings. New York, NY: Free Press.

Alter, K. (2007). Social enterprise typology. Virtue Ventures LLC, 12, 1-124.

Alvarez, K., Salas, E., \& Garofano, C. M. (2004). An integrated model of training evaluation and effectiveness. Human resource development Review, 3(4), 385416.

Ankli, R. E., \& Palliam, R. (2012). Enabling a motivated workforce: exploring the sources of motivation. Development and Learning in Organizations: An International Journal, 26(2), 7-10. 
Arthur, W., Day, E.A., Bennett, W., McNelly, T. L., \& Jordan, J. A. (1997). Dyadic versus individual training protocols: loss and reacquisition of a complex skill. Journal of Applied Psychology, 82,783-791.

Aycan, Z., \& Berry, J. W. (1996). Impact of employment-related experiences on immigrants' psychological well-being and adaptation to Canada. Canadian Journal of Behavioral Science, 28(3), 240-251.

Bakewell, O. (2003). Community services in refugee aid programmes: a critical analysis. New Issues in Refugee Research, Working Paper no. 82, UNHCR, Geneva, 2003.

Bakker, L., Dagevos, J., \& Engbersen, G. (2016). Explaining the refugee gap: A longitudinal study on the labour market participation of refugees in the Netherlands. Journal of Ethnic and Migration Studies, 43, 1775-1791.

Bandura, A. (2006). Toward a Psychology of Human Agency. Retrieved from Stanford University website: https://pdfs.semanticscholar.org/4c53/0339369cff0093101ba611b28e48620f3493. pdf

Bandura, A. (1977a). Social learning theory. Englewood Cliffs, NJ: Prentice Hall. Bandura, A. (1977b). Self-efficacy: Toward a unifying theory of behavior change. Psychological Review, 84, 191-215.

Bandura, A. (1982). Self-efficacy mechanism in human agency. American Psychologist, $37,122-147$.

Bandura A. (1986). Social Foundations of Thought and Action: A Social Cognitive Theory. Englewood Cliffs, NJ: Prentice-Hall. 
Bandura, A. (1997). Self-efficacy: The exercise of control. New York, NY: W.H Freeman.

Bandura, A., \& Locke, E. A. (2003). Negative self-efficacy and goal effects revisited. Journal of Applied Psychology, 88(1), 87-99.

Battilana, J., Lee, M., Walker, J., Dorsey, C., \& Mendelsohn, D. (2012). In Search of the Hybrid Ideal. Retrieved from Stanford University website: http://www.ssireview.org/articles/entry/in_search_of_the_hybrid_ideal

Baumhof, R., Decker, T., Roder, H., \& Menrad, K. (2017). An expectancy theory approach: What motivated and differentiates German house owners in the context of energy efficient refurbishment measures? Energy and Buildings, 152(2017), 483-491.

Baxter, P., \& Jack, S. (2008). Qualitative case study methodology: Study design and implementation for novice researchers. The Qualitative Report, 13(4), 544-559.

Becker, P. C., \& Isaacs, J. (1996). A new American acculturation study five years later. Journal of Jewish Communal Service, 72, 360-398.

Bereby-Meyer, Y., \& Kaplan, A. (2005). Motivational influences on transfer of problemsolving strategies. Contemporary Educational Psychology, 30,1-22.

Bloch, A. (2002). The migration and settlement of refugees in Britain. Basingstoke and London: Palgrave Macmillan.

Bloch, A. (2004). Making it work: refugee employment in the UK (Vol. 2). Institute for Public Policy Research.

Bloch, A., Galvin, T., \& Harrell-Bond, B. (2000). Refugee women in Europe: Some aspects of the legal and policy dimensions. International Migration, 38, 169-190. 
Bornstein, D. (2004). How to change the world: Social entrepreneurship and the power of ideas. Oxford University Press. New York: NY.

Bowen, A. (2016, November 22). Refugee women find friendship, income in making scarves for Chicagoans. Chicago Tribune. Retrieved from http://www.chicagotribune.com/lifestyles/ct-refugees-loom-scarves-family-111420161122-story.html

Boyd, B., N. Henning, N., Reyna, E., Wang, D. E., Welch, M. D., \& Hoffman, A. J. (2009) Hybrid Organizations: New Business Models for Environmental Leadership. Greenleaf Publishing: Sheffield.

Brissenden, P. (1925). Political Science Quarterly, 40(3), 472-476. doi:10.2307/2142220

Bruce, T. C. (2006). Contested Accommodation on the Meso Level Discursive Adaptation Within Catholic Charities' Immigration and Refugee Services. American Behavioral Scientist, 49(11), 1489-1508.

Buckingham, S., Marandet, E., Smith, F., Wainwright, E., \& Diosi, M. (2006). The liminality of training spaces: places of private/public transitions. Geoforum, 37(6), 895-905.

Bulcha, M. (1988). Flight and integration: Causes of mass exodus from Ethiopia and problems of integration in the Sudan. Nordic Africa Institute.

Burke, M. (2016, October, 4). 6 immigrant stories that will make you believe in the American dream again. Retrieved from https://www.forbes.com/sites/monteburke/2016/10/04/6-immigrant-stories-thatwill-make-you-believe-in-the-american-dream-again/\#468b434a8027 
Bodnar, J. (1980). Immigration, kinship, and the rise of working-class realism in industrial America. Journal of Social History, 14(1), 45-65.

Caligiuri, P., Philips, J., Lazarova, M., Tarique, I., \& Burgi, P. (2001). The theory of met expectations applied to expatriate adjustment: the role of cross-cultural training. International Journal of Human Resource Management, 12, 357-372.

Cameron, H. (2004). The Nonprofit Phenomenon. Searcher, 12(2), 33-41.

Campion, E. D. (2018). The career adaptive refugee: Exploring the structural and personal barriers to refugee resettlement. Journal of Vocational Behavior, 105, 616.

Candela, L., Gutierrez, A. P., \& Keating S. (2015). What predicts nurse faculty members' intent to stay in the academic organization? A structural equation model of a national survey of nursing faculty. Nurse Education Today, 35(4), 580-589.

Capps, R., \& Newland, K. (2015). The Integration Outcomes of U.S. Refugees: Successes and Challenges. Washington, DC: Migration Policy Institute.

Carpenter, J. P., Daniere, A. G., \& Takahashi, L. M. (2004). Social capital and trust in South-east Asian cities. Urban Studies, 41(4), 853-874.

Casimiro, S., Hancock, P., \& Northcote, J. (2007). Isolation and insecurity resettlement issues among muslim refugee women in Perth, Western Australia. The Australian Journal of Social Issues, 42(1), 55-69. doi:10.1002/j.1839-4655.2007.tb00039.x

Cercone, K. (2008). Characteristics of adult learners with implications for online learning design. AACE journal, 16(2), 137-159.

Champion, M.R. (2008). Creating engagement: The use of expectancy theory in corporate customer service teams. Dissertation. Capella University. 
Charles, G., Bainbridge, L., \& Gilbert, J. (2004). Interprofessional education and practice in the health and human services: The University of British Columbia Model. Vancouver, British Columbia: UBC.

Chen, G., Gully, S., \& Eden, D. (2004). General self-efficacy and self-esteem: Towards theoretical and empirical distinction between correlated self-evaluations. Journal of Organizational Behavior, 25(3), 375-395.

Cheng, E. W. L., \& Ho, D. C. K. (2001). A review of transfer of training studies in the past decade. Personnel Review, 30, 102-118.

Child Welfare Information Gateway. (2013). Leaving your child home alone. Washington, DC: U.S. Department of Health and Human Services, Children's Bureau.

Chishti, M., Hipsman, F., \& Ball, I. (2015, October 15). Fifty years on, the 1965 Immigration and Nationality Act continues to reshape the United States. Retrieved from https://www.migrationpolicy.org/article/fifty-years-1965immigration-and-nationality-act-continues-reshape-united-states

Colic-Peisker, V. (2009). Visibility, settlement success and life satisfaction in three refugee communities in Australia. Ethnicities, 9(2), 175-199.

Colic-Peisker, V., \& Tilbury, F. (2006). Employment niches for recent refugees: Segmented labour market in twenty-first century Australia. Journal of Refugee Studies, 19(2), 203-229.

Colquitt, J. A., \& Simmering, M. S. (1998). Conscientiousness, goal orientation, and motivation to learn during the learning process: A longitudinal study. Journal of Applied Psychology, 83, 654-665. 
Correa-Velez, I., Gifford, S., \& Barnett, A. (2010). Longing to belong: Social inclusion and well-being among youth with refugee backgrounds in the first three years in Melbourne, Australia. Social Science \& Medicine, 17(8), 1399-1408. doi:10.1016/j.socscimed.2010.07.018

COSMOS Corporation. (1989, September). Attracting high-technology firms to local areas:Lessons from nine high-technology and industrial parks. Bethesda, MD

Cowan, J. (2014). Noteworthy matters for attention in reflective journal writing. Active Learning in Higher Education, 15, 53-64. doi:10.1177/1469787413514647

De Corte, E. (2003). Transfer as the productive use of acquired knowledge, skills, and motivations. Current Directions in Psychological Science, 12, 142-146.

Deem, J. M., \& Marshall, W. J. (1980). Teaching a second language to Indochinese refugees when no program exists. Journal of Reading, 23(7), 601-605.

DeNavas-Walt, C., \& Proctor, B. D. (2014). Income and Poverty in the United States: 2014. Retrieved from https://census.gov/content/dam/Census/library/publications/2015/demo/p60252.pdf

Department of Homeland Security. (2017). Refugees \& asylum. Retrieved from https://www.uscis.gov/humanitarian/refugees-asylum

De Vriese, M. (2006). Refugee Livelihoods: A review of the evidence. UNHCR, EPAU/2006/04, Geneva, 2006.

Dickson, M. A., \& Littrell, M. A. (1998). Organizational culture for small textile and apparel businesses in Guatemala. Clothing and Textiles Research Journal, 16(2), 68-78. 
Dinibutun, S. R. (2012). Work motivation: Theoretical framework. GSTF Business Review (GBR), 1(4), 133.

Doherty, B., Haugh, H., \& Lyon, F. (2014). Social enterprises as hybrid organizations: A review and research agenda. International Journal of Management Reviews, $16(4), 417-436$.

Fangen, K. (2006). Humiliation Experienced by Somali Refugees in Norway, Journal of Refugee Studies, 19(1), 69-93.

Feltz, D.L., Short, S. \& Sullivan, P. (2008). Self-efficacy in sport: Research and strategies for working with athletes, teams and coaches. Champaign, IL: Human Kinetics. doi:10.1260/174795408785100699

Feltz, K.L. \& Mugno, D.A. (1983) A replication of the path analysis of the causal elements in Bandura's theory of self-efficacy and the influence of automatic perception. Journal of Sport Psychology, 5, 263-277.

Finch, C. R., \& Crunkilton, J. R. (1999). Curriculum development in vocational and technical education. Planning, content, and implementation. Allyn and Bacon, 160 Gould Street, Needham Heights, MA 02494.

Finn, H. B. (2010). Overcoming barriers: Adult refugee trauma survivors in a learning community. TESOL Quarterly, 44(3), 586-596.

Finann, C. R. (1981). Occupational assimilation of refugees. The International MigrationReview, 15(1/2), 292-309.

Ford, M.E. (1992). Motivating humans: Goals, emotions, and personal agency beliefs. Newbury Park, CA: Sage. 
Fozdar, F. (2012). Social cohesion and skilled Muslim refugees in Australia Employment, social capital and discrimination. Journal of Sociology, 48(2), $167-$ 186.

Freire, M. (1990). Refugees: ESL and Literacy Trying to Reinvent the Self in a New Language. Refuge: Canada's Journal on Refugees, 10(2).

Gambrel, P. A., \& Cianci, R. (2003). Maslow's hierarchy of needs: Does it apply in a collectivist culture. Journal of Applied Management and Entrepreneurship, 8(2), $143-161$

Gegenfurtner, A., Veermans, K., Festner, D., \& Gruber, H. (2009). Motivation to transfer training: An integrative literature review. Human Resource Development Review, 8, 403-423.

Gist, M. E., \& Mitchell, T. R. (1992). Self-efficacy: A theoretical analysis of its determinants and malleability. Academy of Management Review, 17, 183-211.

Gist, M. E., Stevens, C. K., \& Bavetta, A. G. (1991). Effects of self-efficacy and posttraining intervention on the acquisition and maintenance of complex interpersonal skills. Personnel Psychology, 44, 837- 861.

Goldin, C., \& Sokoloff, K. (1982). Women, children, and industrialization in the early republic: Evidence from the manufacturing censuses. The Journal of Economic History, 42(4), 741-774.

Goldschein, E. (2012, February 1). 13 people who came to America with nothing and made a fortune. Retrieved from http://www.businessinsider.com/came-toamerica-with-nothing-and-made-a-fortune-2012-1 
Goodman, R. D., Vesely, C. K., Letiecq, B., \& Cleaveland, C. L. (2017). Trauma and resilience among refugee and undocumented immigrant women. Journal of Counseling \& Development, 95, 309-321. doi:10.1002/jcad.12145

Goworek, H. (2011). Social and environmental sustainability in the clothing industry: a case study of a fair trade retailer. Social Responsibility Journal, 7(1), 74-86.

Harrell-Bond, B. E. (1986). Imposing aid: emergency assistance to refugees (p. 15). Oxford: Oxford University Press.

Hassanin, L. (2008, June). Egyptian women artisans: Struggling to face the demands of modern markets. In Technology and Society, 2008. ISTAS 2008. IEEE International Symposium on (pp. 1-8). IEEE.

Hayton, J. C., \& Cholakova, M. (2012). The role of affect in the creation and intentional pursuit of entrepreneurial ideas. Entrepreneurship: Theory and Practice, 36(1), 41-68.

Hofstede, G. (1980). Motivation, leadership, and organization: Do American theories apply abroad. Organizational Dynamics. 9 (1). $42-63$.

Hofstede, G. (1983). The culture relativity of organizational practices and theories. Journal of International Business Studies. 14 (2), 75-89.

Holloway, C. M. (1976). Cuban refugee and the American dream. Change, 8(9), 11-13.

Holton, E. F. (1996). The flawed four-level evaluation model. Human Resource Development Quarterly, 7, 5-21.

Holton, E. F., Bates, R. A., \& Ruona, W. E. A. (2000). Development and construct validation of a generalized learning transfer system inventory. Human Resource Development Quarterly, 11(4), 333-360. 
Hoogendoorn, B., Pennings, E., \& Thurik, R. (2010). What do we know about social entrepreneurship: An analysis of empirical research.

Howard, H., Nazari, M., \& De Leal, A. (2006). Living as a Refugee in America: Mohammed's Story. Gareth Stevens.

Hynes, B. (2009). Growing the social enterprise-issues and challenges. Social Enterprise Journal, 5(2), 114-125.

Ingram, D. (2013). Non Profit Organization Vs. Profit Organization.

Igielnik, R., \& Krogstad, J. M. (2017). Where refugees to the U.S. come from. Retrieved from http://www.pewresearch.org/fact-tank/2017/02/03/where-refugees-to-the-us-come-from/

Ivy, J. (2008). A new higher education marketing mix: the 7Ps for MBA marketing. International Journal of Educational Management, 22(4), 288299. doi.org/10.1108/09513540810875635

Johnston, J. H., \& Cannon-Bowers, J. A. (1996). Training for stress exposure. In J. E. Driskell \& E. Salas (Eds.), Stress and human performance (pp. 223-256). Mahweh, NJ: Erlbaum.

Kaiser, T. (2001). A beneficiary-based evaluation of UNHCR's programme in Guinea, West Africa. UNHCR, EPAU/2001/02, Geneva, 2001.

Kanfer, R. (1991). Motivation theory and industrial and organizational psychology. In M. D. Dunnette \& L. M. Hough (Eds.), Handbook of industrial and organizational psychology (Vol. 1, pp. 75-170). Palo Alto, CA: Consulting Psychologists Press. Kanter, R. M. (2006). Confidence: How winning and losing streaks begin and end. New York, NY: Crown Publishing. 
Kavanagh, D.J. \& Bower, G.H. (1985). Mood and self-efficacy: Impact of joy and sadness on perceived capabilities. Cognitive Therapy and Research, 9(5), 507525. doi:10.1007/BF01173005.

Keiler, P. (2017, September 19). Extraordinary campaign underway at Glen Ellyn boutique: Women supporting women. Chicago Tribune. Retrieved from http://www.chicagotribune.com/suburbs/elmhurst/community/chi-ugc-articleextraordinary-campaign-underway-at-glen-ellyn-1-2017-09-19-story-html

Kerlin, J. A. (2006). Social enterprise in the United States and Europe: Understanding and learning from the differences. Voluntas: International Journal of Voluntary and NPOs, 17(3), 246.

Kernot, C., \& McNeil, J. (2011). Australian stories of social enterprise. Sydney, Australia: University of New South Wales.

Kirk, A., Fisk, A. D., Walker, N., \& Rothrock, L. (1998). Feedback augmentation and part-task practice in training dynamic decision-making skills.

Kilpatrick, S., \& Abbott-Chapman, J. (2002). Rural young people's work/study priorities and aspirations: The influence of social capital. Australian Education Researcher, 29(1), 43-68.

Kleinginna, P. R., \& Kleinginna, A. M. (1981). A categorized list of motivation definitions, with a suggestion for a consensual definition. Motivation and emotion, 5(3), 263-291.

Knowles, M. (1984). The Adult Learner: A Neglected Species (3rd Ed.). Houston, TX: Gulf Publishing. 
Knowles, M. S., Holton, E. F., \& Swanson, R. A. (2005). The adult learner-The definitive classic in adult education and human resource development. Amsterdam: Elsevier.

Kogan, I. (2003). Ex-Yugoslavs in the Austrian and Swedish labour markets: The significance of the period of migration and the effect of citizenship acquisition. Journal of Ethnic and Migration Studies, 29, 595-622.

Lamba, N. K. (2003). The employment experiences of Canadian refugees: Measuring the impact of human and social capital on quality of employment. Canadian Review of Sociology and Anthropology, 40(1), 45-64.

Levine, L. (1924). The Women's Garment Workers: A History of the International Ladies' Garment Worker's Union. BW Huebsch.

Li, P. S. (2000). Earning disparities between immigrants and native-born Canadians. The Canadian Review of Sociology and Anthropology, 37(2), 289-311.

Lincoln, Y. S., \& Guba, E. G. (1985). Naturalistic Inquiry. Newbury Park, CA: Sage Publications.

Liptak, J.J. (2008). Career quizzes. Indianapolis, IN: JIST Publishing Lipsky, S., \& Nimol, K. (1993). Khmer women healers in transition: Cultural and bureaucratic barriers in training and employment. Journal of Refugee Studies, 6(4), 372-388.

Lloyd, R., \& Mertens, D. (2018). Expecting more out of expectancy theory: History urges inclusion of the social context. International Management Review, 14(1), 24-37.

Littrell, M., \& Dickson, M. (1997). Alternative trading organizations: Shifting paradigm in a culture of social responsibility. Human Organization, 56(3), 344-352. 
Lunenburg, F. C. (2011a). Self-efficacy in the workplace: implications for motivation and performance. International journal of management, business, and administration, 14(1), 1-6.

Lunenburg, F. C. (2011b). Theorizing about Curriculum: Conceptions and definitions. International Journal of Scholarly Academic Intellectual Diversity, 13(1), 1-6.

Marger, M. N. (2006). Transnationalism or assimilation? Patterns of sociopolitical adaptation among Canadian business immigrants. Ethnic and Racial Studies, 29(5), 882-900.

Marshall, C., \& Rossman, G. (1989). Designing qualitative research. Newbury Park/London/New Delhi.

Martella, D., \& Maass, A. (2000). Unemployment and life satisfaction: The moderating role of time structure and collectivism. Journal of Applied Social Psychology, 30, 1095-1108.

Martocchio, J. J., \& Webster, J. (1992). Effects of feedback and cognitive playfulness on performance in microcomputer training. Personnel Psychology, 45, 553-578.

Maslow, A. H. (1970). Motivation and personality. New York: Harper \& Row.

Massey, D. S. (1995). The new immigration and ethnicity in the United States. Population and Development Review, 631-652.

Massey, D. S. (1999). The handbook of international migration: The American experience. New York: Russel Sage Foundation.

Mathieu, J. E., Tannenbaum, S. I., \& Salas, E. (1992). Influences of individual and situational characteristics on measures of training effectiveness. Academy of Management Journal, 35, 828-847. 
Mathieu J. E., \& Martineau, J. W. (1997). Individual and situational influences in training motivation. In J. K. Ford, S. W. J., Kozlowski, K. Kraiger, E. Salas, \& M. S. Teachout (Eds.). Improving training effectiveness in work organizations (pp. 193222). New York, NY: Lawrence Erlbaum Associates, Inc.

Maynard, D. C., \& Ferdman, B. M. (2009). The marginalized workforce: How IO psychology can make a difference. The industrial-organizational psychologist, 46(4), 25-29.

McKee, S.L., \& Walters, B.L. (2002). Transition management: A practical approach to personal and professional development. Upper Saddle River, NJ: Pearson Education.

Merriam, S. B. (1998). Qualitative Research and Case Study Applications in Education. Revised and Expanded from" Case Study Research in Education." Jossey-Bass Publishers, 350 Sansome St, San Francisco, CA 94104.

Miles, B. M., Huberman, A. M., \& Saldaña, J. (2014). Qualitative data analysis (3rd ed.). Los Angeles: Sage.

Miller, K., \& Billings, D. (1994). Playing to grow: A primary mental health intervention with Guatemalan refugee children. American Journal of Orthopsychiatry, 64(3), 346-356.

Morrice, L. (2007). Lifelong learning and the social integration of refugees in the UK: The significance of social capital. International Journal of Lifelong Education, 26(20), 155-172.

Mott, T. E. (2010). African refugee resettlement in the US: the role and significance of voluntary agencies. Journal of Cultural Geography, 27(1), 1-31. 
Neilsen, S. R. (2015, June 18). Texas refugees create a new line for Ikea. Houstonia. Retrieved from https://www.houstoniamag.com/articles/2015/6/18/texas-refugeescreate-a-new-line-for-ikea-june-2015

Nevis, B.C. (1983). Using an American perspective in understanding another culture: Toward a hierarchy of needs for the People's Republic of China. The Journal of Applied Behavioral Science, 19(3), 249-264.

Negy, C., Schwatrz, S., \& Reig-Ferrer, A. (2009). Violated expectations and acculturative stress among US Hispanic immigrants. Cultural Diversity and Ethnic Minority Psychology, 15(3), 255-264.

Nijman, D. J. J. M. (2004). Supporting transfer of training: Effects of the supervisor. Enshede: Universiteit Twente.

Noe, R. A. (1986). Trainees' attributes and attitudes: Neglected influences on training effectiveness. Academy of Management Review, 11,736-749.

Noe, R. A., \& Schmitt, N. (1986). The influence of trainee attitudes on training effectiveness: Test of a model. Personnel psychology, 39(3), 497-523.

Norman, G. R. (1999). The adult learner: A mythical species. Academic Medicine, 74, 886-889.

OECD (2005). Promoting adult learning: Education and training policy. Paris: OECD

O'Hara, P. (2001). Social Enterprises and local development. The emergence of social. In Borzaga, C. and Defourney, J. (Eds.), Routledge, London.

Olliff, L. (2010). What works: employment strategies for refugee and humanitarian entrants. Sydney: Refugee Council of Australia. 
Otto, K., \& Dalbert, C. (2012). Individual differences in job-related relocation readiness: The impact of personality dispositions and social orientations. Career Development International, 17, 168-186.

Park, S., \& Kim, S. (2017). The linkage between work unit performance perceptions of U.S. federal employees and their job satisfaction: an expectancy theory. Transylvania Review of Administrative Sciences, 52, 77-93.

Page-Reeves, J. (1998). Alpaca sweater design and marketing: problems and prospects for cooperative knitting organizations in Bolivia. Human Organization, 57(1), 8393.

Patrick, J. (2000). Training. In N. Chmiel (Ed.), Introduction to work and organizational psychology (pp. 100-125). Oxford, UK: Blackwell.

Pajares, M.F. (1992). Teacher beliefs and educational research: Cleaning up a messy construct. Review of Educational Research, 62, 307-332.

Pattinson, E. M., Cotterill, S. T., \& Leyland, S. D. (2017). Sources of self-efficacy in springboard and highboard diving: A qualitative investigation. Sport \& Exercise Psychology Review, 13(1), 80-91.

Patton, M. Q. (1990). Qualitative evaluation and research methods (2nd ed.). London: Sage.

Perez, A. D., \& Hirschman, C. (2009). The Changing Racial and Ethnic Composition of the US Population: Emerging American Identities. Population and Development Review, 35(1), 1-51. doi.org/10.1111/j.1728-4457.2009.00260.x

Pfeffer, J. (1994). Competitive advantage through people: Unleashing the power of the workforce. Boston, MA: Harvard Business School Press. 
Pfeffer, J., \& Veiga, J. (1999). Putting people first for organizational success. Academy of Management Executive, 13, 37-48.

Pitimaneeyakul, U., LaBat, K. L., \& DeLong, M. R. (2004). Knitwear product development process: A case study. Clothing and Textiles Research Journal, 22(3), 113-121.

Portes, A. (1969). Dilemmas of a Golden Exile: Integration of Cuban Refugee Families in Milwaukee. American Sociological Review, 34(4), 511-515.

Quinones, M. A. (1995). Pretraining context effects: Training assignment as feedback. Journal of Applied Psychology, 80, 226-238.

Raemdonck, I. (2006). Self-directedness in learning and career processes: A study in lower-qualified employees in Flanders. Gent: Universiteit van Gent.

Ramlall, S. (2004). A review of employee motivation theories and their implications for employee retention within organizations. Journal of American Academy of Business, 5(1/2), 52-63.

Reddy, J. (2014, July 30). UK fashion social enterprises support recovering addicts, refugees and ageing. Retrieved July 7, 2016, from https://www.theguardian.com/sustainable-business/sustainable-fashion-blog/uk$\underline{\text { social-enterprise-fashion-support-refugees-women }}$

Refugee Council USA. (2018). History of the U.S. Refugee Resettlement Program. Retrieved from https://www.rcusa.org/history/

Reuss, A. (2009). That 70's Crisis. Retrieved July 21, 2016, from http://www.dollarsandsense.org/archives/2009/1109reuss.html 
Rischin, M. (1962). The Promised City: New York's Jews, 1870-1914 (Vol. 120). Harvard University Press.

Roller, M. R., \& Lavrakas, P. J. (2015). Applied qualitative research design: A total quality framework approach. Guilford Publications.

Rose, R. (1991). Comparing forms of comparative analysis. Political studies, 39, 446-462

Roy, K., Zvonkovic, A., Goldberg, A., Sharp, E., \& LaRossa, R. (2015). Sampling richness and qualitative integrity: Challenges for research with families. Journal of Marriage and Family, 77(1), 243-260.

Rupasingha, A., Ilvento, T. W., \& Freshwater, D. (2000). Demand for Skills Training in The Rural South. University of Kentucky, Department of Agricultural Economics.

Rouiller, J. Z., \& Goldstein, I. L. (1993). The relationship between organizational transfer climate and positive transfer training. Human Resource Development Quarterly, 4(4), 377-390.

Saldaña, J. (2013). The coding manual for Qualitative Researchers, $2^{\text {nd }}$ edition. Los Angeles: Sage Publications.

Saez, R. (2017, July 17). With Muses, immigrants and refugees sew a fresh start. Seattle Met. Retrieved from https://www.seattlemet.com/articles/2017/7/17/with-musesimmigrants-and-refugees-sew-a-fresh-start

Salas, E., \& Cannon-Bowers, J. A. (2001). The Science of Training: A decade of progress. Annual Reviews Psychology, 52, 471-499.

Schepman, S. B., \& Richmond, L. (2003). Employee expectations and motivations: An application form the "learned helplessness" paradigm. Journal of American Academy of Business, 3, 405. 
Schlossberg, N. K. (1981). A model for analyzing human adaptation to transition. The Counseling Psychologist, 9, 2-18.

Schmidt, S. W. (2007). The relationship between satisfaction with workplace training and Overall job satisfaction. Human Resource Development Quarterly, 18(4), 481498.

Schreiner, M. (2014). Mirroring. Retrieved from https://evolutioncounseling.com/mirroring/

Shute, V.J., \& Gawlick, L. A. (1995). Practice effects on skill acquisition, learning outcome, retention, and sensitivity to relearning. Human Factors, 37, 781-803.

Smith, L. R. (1996). Intercultural social support networks: A socio-structural description of expatriate adjustment and communicative competence (Unpublished doctoral dissertation). University of Minnesota, Minneapolis.

Smith, A.D., \& Rupp, W.T. (2004). Managerial challenges of e-recruiting: extending the life cycle of new economy employees. Online Information Review, 28(1), 61-74.

Sienkiewicz, H. C., Mauceri, K. G., Howell, E. C., \& Bibeau, D. L. (2013). Untapped resources: Refugee employment experiences in Central North Carolina. Work, 45, 17-24. doi: 10.3233/WOR-131599

Stajkovic, A. D., \& Luthans, F. (1998). Self-efficacy and work-related performance: A meta-analysis. Psychological Bulletin, 124, 240-261.

Strawn, S., \& Littrell, M. A. (2006). Beyond capabilities: A case study of three artisan enterprises in India. Clothing and Textiles Research Journal, 24(3), 207-213. 
Stuckey, H. L. (2013). Three types of interviews: Qualitative research methods in social health. Journal of Social Health \& Didactics, 1(2), 56-59. doi:10.4103/23210656.115294.

Suarez-Orozco, J. (2013). What shapes the Experience of Immigrants and their children? Retrieved on https://www.radcliffe.harvard.edu/news/radcliffemagazine/considering-immigration-and-gender-in-americas

Tannenbaum, S. I., Cannon-Bowers, J. A., Salas, E., \& Mathieu, J. E. (1993). Factors that influence training effectiveness: A conceptual model and longitudinal analysis. Orlando, FL: Naval Air Warfare Center Training Systems Divisions.

Taylor, M. S., Giannantonio, C. M. (1990). Vocational Guidance. International Review of Industrial and Organizational Psychology, 5, 281-323.

Tomlinson, F., \& Egan, S. (2002). From marginalization to (dis) empowerment: Organizing training and employment services for refugees. Human Relations, 55(8), 1019-1043.

Tomlinson, F. (2010). Marking difference and negotiating belonging: Refugee women, volunteering and employment. Gender, Work \& Organization, 17(3), 278-296.

Tol, W., Komproe, I., Susanty, D., Jordans, M., Macy, R., \& de Jong, J. (2008). Schoolbased mental health intervention for children affected by political violence in Indonesia. Journal of the American Medical Association, 300(6), 655-62.

Toth, J. (2003). Resilience: The experience of immigrant and refugee women. (Master Thesis, University of Manitoba, Manitoba Heritage Thesis Database). Retrieved from http://mspace.lib.umanitoba.ca/handle/1993/3801 
Tshabangu-Soko, T. S., \& Caron, R. M. (2011). English for speakers of other languages (ESOL): Improving English language acquisition for preliterate and nonliterate adult African refugees.

The UN Refugee Agency (UNHCR). (2017a). Figures at a glance. Retrieved from http://www.unhcr.org/en-us/figures-at-a-glance.html

The UN Refugee Agency (UNHCR). (2017b). Left behind: Refugee Education in crisis. Retrieved from http://www.unhcr.org/left-behind/

U.S. Citizenship \& Immigration Services. (2015). Refugees \& Asylum. Retrieved from https://www.uscis.gov/humanitarian/refugees-asylum

U.S. City Populations 2018. (n.d.). In World Population Review. Retrieved from http://worldpopulationreview.com/us-cities/

U.S. Department of Health \& Human Services. (1979). The Belmont report. Retrieved from http://www.hhs.gov

U.S. Department of Health \& Human Services. (2015). Office of Refugee Resettlement: Fiscal Year 2014 Refugee Arrivals. Retrieved from http://www.acf.hhs.gov/programs/orr/resource/fiscal-year-2014-refugee-arrivals

U.S. Department of Health \& Human Services. (2017). Office of refugee resettlement: An office of the administration for children \& families. Retrieved from https://www.acf.hhs.gov/orr/resource/national-voluntary-agency-directory

U.S. Department of Health \& Human Services. (2017). Office of refugee resettlement: What we do. Retrieved from https://www.acf.hhs.gov/orr/about/what-we-do 
U.S. Department of Health \& Human Services. (2018). U.S. federal poverty guidelines used to determine financial eligibility for certain federal programs. Retrieved from https://aspe.hhs.gov/poverty-guidelines

U.S. Department of State. (2017). Refugee admissions statistics. Retrieved from https://www.state.gov/j/prm/releases/statistics/index.htm

Valtonen, K. (2004). From the margin to the mainstream: Conceptualizing refugee resettlement process. Journal of Refugee Studies, 17, 70-96.

van Kooy, J. (2016). Refugee women as entrepreneurs in Australia. Forced Migration Review, 53, 71-73.

Victorian Settlement Planning Committee (VSPC). (2008). Pathways and transitions: post-compulsory education, work and refugee young people. Centre for Multicultural Youth, Melbourne, Victoria.

Vroom, V. H. (1964). Work and motivation. New York: Wiley.

Vroom, V. H. (1995). Work and Motivation, Revised Edition, Jossey-Bass Classics

Vroom, V. H. (2018). In Yale School of Management:Faculty Research \& Centers. Retrieved from https://som.yale.edu/faculty/victor-h-vroom

Wagnild, G., \& Collins, J. (2009). Assessing resilience: Journal of Psychosocial Nursing, 47(12), 28-33. doi:10.3928/02793695-20091103-01

Walter, I. (1981). One year after arrival, the adjustment of Indochinese Women in the United States. New York: Lutheran Immigration and Refugee Services.

Ward, C., Bochner, S., \& Furnham, A. (2006). The psychology of culture shock (2nd ed.). New York: Routledge. 
Watkins, P. G., Razee, H., \& Richters, J. (2012). 'I'm Telling You... The Language Barrier is the Most, the Biggest Challenge': Barriers to Education among Karen Refugee Women in Australia. Australian Journal of Education, 56(2), 126-141.

Woodgate, J. \& Brawley, L.R. (2008). Use of an efficacy-enhancing message to influence the self-regulatory efficacy of cardiac rehabilitation participants: A field experiment. Rehabilitation Psychology, 53(2), 153. doi: 10.1037/00905550.53 .2 .153

Workforce Training and Education Coordinating Board. (2008). High Skills, High Wages 2008-2018: Washington's Strategic Plan for Workforce Development. Retrieved from https://www.wsac.wa.gov/sites/default/files/HighSkillsHighWages-WTB2008.pdf

Yin, R. K. (1994). Case study research: Design and methods (2nd ed.). Newbury Park, CA: Sage Publications

Yin, R. K. (2014). Case Study Research: design and methods ( $5^{\text {th }}$ ed.). Los Angeles: Sage Publications.

Yin, R. K. (2009). Case Study Research: design and methods. ( ${ }^{\text {th }}$ ed.). Los Angeles, CA: Sage Publications.

Yin, R. K. (2012). Applications of case study research. ( $3^{\text {rd }}$ ed.). Los Angeles, CA: Sage Publications.

Zhou, M. (1997). Segmented assimilation: Issues, controversies, and recent research on the new second generation. International migration review, 975-1008.

Zong, J., \& Batalova, J. (February 26, 2015). Frequently requested statistics on immigrants and immigration in the United States. Retrieved on July 21, 2015 
from: http://www.migrationpolicy.org/article/frequently-requested-statisticsimmigrants-and-immigration-united-states

Zong, J. \& Batalova, J. (2017, March 8). Frequently requested statistics on immigrants and immigration in the United States. Retrieved from https://www.migrationpolicy.org/article/frequently-requested-statisticsimmigrants-and-immigration-united-states. 


\title{
APPENDIX A
}

\author{
CAREER TRANSITIONS: A CASE STUDY EXPLORING THE TRAINING
}

EXPERIENCES OF REFUGEE WOMEN IN TEXTILE AND APPAREL SOCIAL

ENTERPRISES

Email Recruitment Letter

Dear

I hope this email finds you well. Let me start my introducing myself, my name is Angela Uriyo and I am PhD Candidate at the University of Missouri, working on my doctoral research in social enterprises training refugee women for careers in the textile and apparel industry.___________________ gave me your contact information as somebody who could potentially connect me with organizations that are in the sector.

Let me tell you a little bit about my research direction:

My doctoral research investigates the career transition experiences of refugee women training in US-based socially enterprises in the textile and apparel industry. Using case study methodology, social enterprises will be explored. I propose to use participant observations and in-depth interviews to understand career transition experiences of refugee women in textile and apparel social enterprises. I will interview refugee women who are past or present participants of these training programs. I will also interview leaders in your organization to better understand the structure of your organization. I am looking for two organizations that are willing to be part of this study.

Please let me know if you have any questions -I appreciate your consideration and look forward to hearing back from you soon.

Sincerely,

\section{Angela Uriyo}

Doctoral Candidate | Textile and Apparel Management

Scholar | The Deaton Institute for University Leadership in International Development

Board Member | Advisory Board of MU African Interdisciplinary Studies Hub

College of Human Environmental Sciences

University of Missouri

137 Stanley Hall

Columbia, MO65211 


\title{
APPENDIX B
}

CONSENT TO ACT AS A HUMAN PARTICIPANT: FOR REFUGEE WOMEN

\author{
Project Title: CAREER TRANSITIONS: A CASE STUDY EXPLORING THE \\ TRAINING EXPERIENCES OF REFUGEE WOMEN IN TEXTILE AND APPAREL
}

SOCIAL ENTERPRISES

Exempt IRB Project Number: 2009859

\section{Project Director: Angela Uriyo \& Pamela Norum}

To participate in this study you must identify with all the following criteria: (1) Have lived in the US as a refugee woman (2) Be an adult female, (3) Be in the process of a career transition (skills training program) or have completed a career transition program (skills training program), and (4) speak conversational English. If you meet all of these criteria, then we would like to invite you to participate in this research study. Your participation is very important to us!

\section{Introduction}

This consent may contain words that you do not understand. Please ask the investigator or the study staff to explain any words or information that you do not clearly understand.

You are being asked to participate in a research study. This research is conducted to gain in-depth knowledge and understanding of career transition experiences of refugee women training in Textile and Apparel Social Enterprises. When you are invited to participate in research, you have the right to be informed about the study procedures so that you can decide whether you want to consent to participation. This form may contain words that you do not know. Please ask the researcher to explain any words or information that you do not understand.

You have the right to know what you will be asked to do so that you can decide whether or not to be in the study. Your participation is voluntary. You do not have to be in the study if you do not want to be. You may refuse to be in the study and nothing will happen. If you do not want to continue to be in the study you may stop at any time without penalty or loss of benefits to which you are otherwise entitled. 


\section{Why is this study being done?}

The purpose of this research is to understand the career transition experiences of refugee women in textile and apparel social enterprises. This research also endeavors to describe the structure of the organizations. The researchers will use in-depth semi structured interview guides and field observation techniques to collect data.

\section{How many people will be in the study?}

10-25 people will take part in this study.

\section{What am I being asked to do?}

Initially you will be asked to participate in a 30 - 40 minute long interview that seeks to assess the role and sources of motivations and agency each participant experiences in making a career transition (going through skills training). This session will be audio-recorded. On completion of the interview, the researchers will transcribe the interview and conduct an analysis of the data collected. Photos will be taken of artifacts that participants may allude to during the interview.

\section{How long will the study last?}

This study will take a total of about six months to complete. Researchers and participants will meet once in a mutually agreed public space such as the organization in which they are going through a career transition (skills training program), for the 30 - 40 minute interview. The researcher will meet with other participants for interviews during this time, and also conduct direct observations which will be recorded in an observational protocol and field notebook.

\section{What are the benefits of being in the study?}

Your participation will benefit social enterprises and refugee resettlement agencies by providing insight to the role and sources of motivations experienced by refugee women when making career transitions via training programs, and you will provide information as to the structure of social enterprises and NPOs in the textile and apparel industry.

\section{What are the risks of being in the study?}

There are no anticipated physical, psychological or sociological risks involved with participating in the study.

\section{What are the costs of being in the study?}

There are no costs to you.

\section{Confidentiality}

The researcher will confidentially save information which is acquired through the interview and direct observations. Any electronic files will be saved with numeric codes, with no personal identifiers. Throughout the procedures, if you feel uncomfortable with any questions or experiences, you may stop participation at any time. Finally, only the researcher will have access 
to the data and the aggregated data will be analyzed and shared for publication. The data will be kept for seven years after the study has been completed.

Information produced by this study will be stored in the investigator's file and identified by a code number only. The code key connecting your name to specific information about you will be kept in a separate, secure location. Information contained in your records may not be given to anyone unaffiliated with the study in a form that could identify you without your written consent, except as required by law.

In addition, if photographs or audiotapes were taken during the study that could identify you, then you must give special written permission for their use. In that case, you will be given the opportunity to view or listen, as applicable, to the photographs, audiotapes or videotapes before you give your permission for their use if you so request.

\section{Will I be compensated for participating in the study?}

You will receive no payment for taking part in this study.

\section{What are my rights as a participant?}

Participation in this study is voluntary. You do not have to participate in this study. You will also be informed of any new information discovered during the course of this study that might influence your health, welfare, or willingness to be in this study.

\section{Who do I contact if I have questions, concerns, or complaints?}

Please contact Angela Uriyo at 573-639-1450 or by e-mail at afup7c@ mail.missouri.edu or Pamela Norum at 573-882-2934 or by email at norump@missouri.edu if you have questions about the research. Additionally, you may ask questions, voice concerns or complaints to the other members of the research team.

\section{Who do I call if I have questions or problems?}

If you have any questions regarding your rights as a participant in this research and/or concerns about the study, or if you feel under any pressure to enroll or to continue to participate in this study, you may contact the University of Missouri Campus Institutional Review Board (which is a group of people who review the research studies to protect participants' rights) at (573) 8829585 or umcresearchcirb@missouri.edu. You may ask more questions about the study at any time. For questions about the study or a research-related injury, contact Angela Uriyo at 573639-1450 or Pamela Norum at 5738822934.

A copy of this Informed Consent form will be given to you before you participate in the research.

\section{SIGNATURES}

I have read this consent form and my questions have been answered. My signature below means that I do want to be in the study. I know that I can remove myself from the study at any time without any problems. 


\author{
APPENDIX C \\ CONSENT TO ACT AS A HUMAN PARTICIPANT: FOR LEADERS IN \\ ORGANIZATIONS
}

Project Title: CAREER TRANSITIONS: A CASE STUDY EXPLORING THE

TRAINING EXPERIENCES OF REFUGEE WOMEN IN TEXTILE AND APPAREL

SOCIAL ENTERPRISES

Exempt IRB Project Number: 2009859

\title{
Project Director: Angela Uriyo \& Pamela Norum
}

To participate in this study you must identify with all the following criteria: (1) be a current instructor, supervisor, director or founder in the organization, and (2) speak English. If you meet all of these criteria, then we would like to invite you to participate in this research study. Your participation is very important to us!

\section{Introduction}

This consent may contain words that you do not understand. Please ask the investigator or the study staff to explain any words or information that you do not clearly understand.

You are being asked to participate in a research study. This research is conducted to gain in-depth knowledge and understanding of career transition experiences of refugee women training in Textile and Apparel Social Enterprises. We are also interested in the structure, vision and mission of your Textile and Apparel Social Enterprise. When you are invited to participate in research, you have the right to be informed about the study procedures so that you can decide whether you want to consent to participation. This form may contain words that you do not know. Please ask the researcher to explain any words or information that you do not understand.

You have the right to know what you will be asked to do so that you can decide whether or not to be in the study. Your participation is voluntary. You do not have to be in the study if you do not want to. You may refuse to be in the study and nothing will happen. If you do not want to continue to be in the study you may stop at any time without penalty or loss of benefits to which you are otherwise entitled.

\section{Why is this study being done?}


The purpose of this research is to understand the career transition experiences of refugee women training in social enterprises. We will also endeavor to describe the structure of the Textile and Apparel Social Enterprises being studied. The researchers will use in-depth semi structured interview guides and direct observation as the primary techniques to collect data.

\section{How many people will be in the study?}

10-25 people will take part in this study.

\section{What am I being asked to do?}

Initially you will be asked to participate in a 30 - 40 minute long interview that seeks to understand the structure, vision and mission of your Textile and Apparel Social Enterprise. This session will be audio-recorded. On completion of the interview, the researchers will transcribe the interview and conduct an analysis of the data collected. Photos may be taken of artifacts that participants may allude to during the interview.

\section{How long will the study last?}

This study will take a total of about six months to complete. Researchers and participants will meet once in a mutually agreed public space such as the organization in which they are going through a career transition (skills training program), for the $30-40$ minute interview. The researcher will meet with other participants for interviews during this time, and also conduct direct observations which will be recorded in an observational protocol and field notebook.

\section{What are the benefits of being in the study?}

Your participation will benefit social enterprises and refugee resettlement agencies by providing insight to the structure, vision and mission of the Textile and Apparel Social Enterprises that refugee women are experiencing career transitions through.

\section{What are the risks of being in the study?}

There are no anticipated physical, psychological or sociological risks involved with participating in the study.

\section{What are the costs of being in the study?}

There are no costs to you.

\section{Confidentiality}

The researcher will confidentially save information which is acquired through the interviews, and direct observations. Any electronic files will be saved with numeric codes, with no personal identifiers. Throughout the procedures, if you feel uncomfortable with any questions or experiences, you may stop participation at any time. Finally, only the researcher will have access to the data and the aggregated data will be analyzed and shared for publication. The data will be kept for seven years after the study has been completed. 
Information produced by this study will be stored in the investigator's file and identified by a code number only. The code key connecting your name to specific information about you will be kept in a separate, secure location. Information contained in your records may not be given to anyone unaffiliated with the study in a form that could identify you without your written consent, except as required by law.

In addition, if photographs or audiotapes were taken during the study that could identify you, then you must give special written permission for their use. In that case, you will be given the opportunity to view or listen, as applicable, to the photographs, audiotapes or videotapes before you give your permission for their use if you so request.

\section{Will I be compensated for participating in the study?}

You will receive no payment for taking part in this study.

\section{What are my rights as a participant?}

Participation in this study is voluntary. You do not have to participate in this study. You will also be informed of any new information discovered during the course of this study that might influence your health, welfare, or willingness to be in this study.

\section{Who do I contact if I have questions, concerns, or complaints?}

Please contact Angela Uriyo at 573-639-1450 or by e-mail at afup7c@mail.missouri.edu or Pamela Norum at 573-882-2934 or by email at norump@ missouri.edu if you have questions about the research. Additionally, you may ask questions, voice concerns or complaints to the other members of the research team.

\section{Who do I call if I have questions or problems?}

If you have any questions regarding your rights as a participant in this research and/or concerns about the study, or if you feel under any pressure to enroll or to continue to participate in this study, you may contact the University of Missouri Campus Institutional Review Board (which is a group of people who review the research studies to protect participants' rights) at (573) 8829585 or umcresearchcirb@missouri.edu. You may ask more questions about the study at any time. For questions about the study or a research-related injury, contact Angela Uriyo at 573639-1450 or Pamela Norum at 5738822934.

A copy of this Informed Consent form will be given to you before you participate in the research.

\section{SIGNATURES}

I have read this consent form and my questions have been answered. My signature below means that I do want to be in the study. I know that I can remove myself from the study at any time without any problems. 


\author{
APPENDIX D \\ CAREER TRANSITIONS: A CASE STUDY EXPLORING THE TRAINING \\ EXPERIENCES OF REFUGEE WOMEN IN TEXTILE AND APPAREL SOCIAL \\ ENTERPRISES
}

\title{
Interview Protocol for Refugee Women who are either Current or Past Trainees
}

These questions are used only for academic research. Your responses will be kept strictly confidential. By answering these questions, you agree that you understand the procedures and any risks and benefits involved in this research. You are free to refuse to participate or to withdraw your consent to participate in this research at any time without penalty or prejudice; your participation is entirely voluntary. Your privacy will be protected because you will not be identified by name as a participant in this project. Your privacy will be protected, as all the information is strictly confidential. If you have any questions concerning your rights as a participant, you may contact Campus Institutional Review Board at 573-882-9585. If you have any questions regarding research, you may contact Angela Uriyo at afup7c@ mail.missouri.edu. Please answer all the questions based on your own personal experiences. Thank you for your time!

\section{PART I: General Intake Questions for ALL refugee women}

1. What is your name?

2. In what year were you born?

3. What is your country of birth?

4. What is your race?

a. What is your ethnicity? Hispanic or Latino/not Hispanic or Latino

5. Do you live alone?

a. If you don't live alone, how many people in your household?

b. If you don't live alone, who do you live with?

6. What is your marital status?

7. Do you have any children?

a. If you do, how many?

b. If you do, are any of your children under the age of 7 years, or require specialized care?

8. What language do you speak most with your family and friends?

9. What languages did you speak in your native country, before coming to the US?

10. In the US, what language do you use most with family and friends?

11. How well did you speak English prior to coming to the US?

12. What have you done to improve your English?

13. Do you/Did you take advantage of free ESL classes to help improve your English?

14. Tell me about your schooling. 
15. What kind of work did you do in your home country to support yourself and your family?

16. In your country, how did you learn the skills that you needed for the work you did?

17. How many years did you work in your occupation in your home country?

18 . What year did you leave your home country?

19. What made you decide to leave your home country (to first or second country of resettlement)?

20. Who travelled with you when you left your home country?

a. If you didn't travel from your home country directly to the US, where did you go and why?

21. What made you decide to come to the US?

22. How did it feel to be separated from your community?

23. Who is back at home that still supports you?

24. How did family member(s) being separated affect your ability to become selfsufficient quickly, (per the immigration law)?

25. Prior to coming to the US, what skills were you told that you needed to get a job in US?

26. Since leaving your country, how have you learned the skills that you believe you need so that you can get a good job in the US?

27. How are you currently supporting yourself/your family?

28. What is your current occupation?

a. Tell me about your employment status. (full-time, part-time, volunteer, home-maker, etc.)

PART II: Questions to Address the central research question: What facilitates or hinders the motivation of women trainees of refugee backgrounds to participate in career transition programs and attain training outcomes?

\section{Question Bank for Current Trainees:}

1. Why are you participating in this training program?

2. Tell me about the community you currently live in, and how they support you?

3. How is your daily life in the US different from when you were in your native country?

4. How is participating in the training program making you feel?

5. What do you believe this training program is doing that will equip you to achieve the "American Dream"?

6. What are some of the things you believe completing this training program will allow you to do for yourself and your family?

7. What did you expect when you started the training program, and what have you experienced so far? Any surprises?

8. What do you expect to learn through the training program that you believe will BEST prepare you for workforce? 
9. What are you currently learning that you believe you will NOT be helpful in helping you find and keep a job?

10. What should this training program really be teaching you that you hear (from others who are working) that would really help you be successful in finding and keeping a job?

11. How often do trainers ask you about what/how they can make the training program better?

-Can you give some examples of recent ideas/suggestions you have given that they have used?

12. How do you believe taking this training programs will help you?

13. How does your teacher grade your work?

14. How are you encouraged to take on harder tasks?

15. How does it make you feel to be part of this training program?

16. How do you expect to feel once you complete this training program?

17. How have people who have taken this training program, and now are working encouraged you to do the same?

18. How do you think completing this training program will help/hinder your ability to find a job?

19. What do you believe this training program is doing that will help you to achieve the "American Dream"?

20. How do you think the training program will improve your ability to work more effectively in an American Company? (soft skills ... communication, punctuality, task completion ... understanding office culture)

21 . What did you have to do to get into the program and what keeps you here?

22. What obstacles or rewards existed before you started the training program, and what role did they play then vs. now?

23. Do you believe that you have the ability to learn the skills and will be able to finish this training program?

24. What are your goals - what do you believe that completing this training program will enable you to do?

25. If something happens and you are not able to complete this training program, what will you not be able to do?

26. What are some things that could stop you from completing this training program?

27. What are some challenges you face that you have to overcome / address in order to keep attending this training program?

28. When you are asked to learn something that is difficult, how does that make you feel (motivated to try hard because this training is important to meeting my goal vs. discouraged because I do not know if it will make a difference)

-Can you give me an example?

29. What effect have other trainees had on your ability to participate in this training program? 
30. What effect do the people giving the training have on your interest level and your desire to participate in the training program?

31. What do potential employers think of these training programs?

32. What do graduates of these programs have to say about how these programs helped them find a new job?

\section{Questions for Graduate Trainees:}

1. Why did you participate in this training program?

2. Tell me about the community you currently live in, and how they support you?

3. How is your daily life in the US different from when you were in your native country?

4. How did participating in the training program make you feel?

5. What do you believe this training program did to train you to achieve the "American Dream"?

6. How did completing the training program affect:

-your life?

-the type of community you live in?

-the type of people you interact with?

-how people treat you or relate to you?

-how your employers treat you?

7. What are some of the things you believed completing this training program will allow you to do for yourself and your family?

8. What are some of the other things you can now do for you and your family that you could not do before completing the training program?

9. What are some of the things you believed completing this training program will allow you to do for yourself and your family?

10. What are some of the other things you can now do for you and your family that you could not do before completing the training program?

11. What did you expect when you started the training program, and what did you experience? Any surprises?

12. How has completing the training program helped you get more job interviews?

13. How have the types of jobs you are applying for changed since completing the training program?

14. What did you expect to learn through the training program that believed would prepare you for the workforce?

15. What did you learn that as a trainee you did not believe was important until you started working?

16. What could the employer/trainer have done to better prepare you for the workforce?

17. How often did trainers ask you about what/how they could make the training program better?

18. Can you give some examples of recent ideas/suggestions you have given that they have used?

19. How has completing this training program made you feel?

20. How did you receive feedback for your work? 
21. How were you encouraged to take on harder task?

22. How have you seen training providers reward people for completing the training program?

23. How has the training program improved your ability to work more effectively in an American Company (soft skills ... communication, punctuality, task completion ... understanding office culture)

24. How did completing the training program affect ... -the type of people you now interact with? -how people treat you or relate to you? -how your employers treat you? 


\section{APPENDIX E \\ CAREER TRANSITIONS: A CASE STUDY EXPLORING THE TRAINING EXPERIENCES OF REFUGEE WOMEN IN TEXTILE AND APPAREL SOCIAL ENTERPRISES}

\section{Interview Protocol for Organizations' Leaders}

The questions on this survey are used only for academic research. Your responses will be kept strictly confidential. By answering the questions on this survey, you agree that you understand the procedures and any risks and benefits involved in this research. You are free to refuse to participate or to withdraw your consent to participate in this research at any time without penalty or prejudice; your participation is entirely voluntary. Your privacy will be protected because you will not be identified by name as a participant in this project. Your privacy will be protected, as all the information is strictly confidential. If you have any questions concerning your rights as a participant, you may contact Campus Institutional Review Board at 573-882-9585. If you have any questions regarding research, you may contact Angela Uriyo at afup7c@mail.missouri.edu. Please answer all the questions. Thank you for your time!

\section{Demographics. Questions to understand who the participant is.}

1. What is your name?

2. Gender

3. Your race: Please specify your race
a. White
b. Black or African American
c. Native American or Alaska native
d. Asian
e. Native Hawaiian or other Pacific Islander
f. Other (please specify)

4. What languages do you speak?

5. Tell me a little bit about your professional and educational background?

6. Employment status at SE: Are you currently...
a. Employed for wages
b. An intern
c. Retiree
d. Unable to work
e. On disability
f. A Volunteer

7. What organization do you work for? (include name of specific training program) 
8. Describe your position in the organization.

9. Tell me why you are working for this organization.

\section{Training Refugee women}

10. What are the biggest challenges/rewards you face as an organization, in working with refugee women? (e.g. funding, trainee retention, staff commitment, location)

11. How do you recruit trainees?

12. How do you keep them engaged and motivated?

13. How do you make them understand what is possible?

14. How do you help them see the end goal?

15. How do you seek to understand what their goals are?

16. What are the challenges that you believe that the women have to overcome to be here?

$$
\text { --e.g. class timing? Why are classes held when they are? }
$$

\section{Defining Success -Training Outcomes}

16. What are you trying to achieve with your training program?

17. How do you monitor progress towards success during the training program (give examples)

18. How do define and measure success for the training programs that you give?

19. What are some of the things that enable success and things that hinder success?

\section{Strategic Alliances}

16.What are the employers saying that they are looking for in your trainees?

17.Are they recruiting your graduates? If not why?

18.What types of strategic alliances does Avenio have with potential employers?

19.In the context of these alliances, have they explored on-the-job training? (to close gaps in training)

20.How does Avenio build trust and credibility with potential employers?

\section{Questions about extrinsic motivation provided to refugee women in the organization}

1. Tell me about the organization's skills training program. (founding, objectives, expected training outcomes, challenges, successes, spin-offs)

2. How does Avenio decide what should go into a training program?

3. Describe the incentives that your SE offers refugee women participating in your training program/workplace.

4. What has worked well, and what gap needs to be closed in your training program?

\section{Questions about intrinsic motivation provided to refugee women in the organization}

5. Describe how this SE empowers the refugee women training or working in the SE to complete or perform work tasks.

6. What do you think are some of the advantages and disadvantages of using a social enterprise to empower women? 


\section{Questions about the Founder/Social Entrepreneur (Ask the Founder of SE ONLY) Questions about what your SE does.}

7. Describe the main income generating activities undertaken by your SE.

8. Explain the barriers that you still face to sustainability and/or growth.

\section{Question about Leadership}

9. Please describe the leadership team of your SE. (numbers, gender, effects of gender on business operation)

\section{Question about Staff}

10. Please describe the staff that currently work for your enterprise.(full-time, part-time, voluntary, capacity, specify gender, immigration status) what is the impact on staff turnover,and how do they affect training program quality?

11. What portion of your SE's funding comes from grants? 0-24\%, 25-49\%, 50-74\%, $75-100 \%$

19. What were some of the significant barriers you encountered when you were starting your social enterprise?

20. What impact has running an SE had on you? (positive and negative)

21. What do you still aspire to do?

22. Is there anything else that you would like to add?

Thank you very much for your time! All data are treated with confidentially. No individuals or organizations will be identified in the final report. 


\section{APPENDIX F \\ Photo Release Form}

Project Title: CAREER TRANSITIONS: A CASE STUDY EXPLORING THE

TRAINING EXPERIENCES OF REFUGEE WOMEN IN TEXTILE AND APPAREL SOCIAL ENTERPRISES

Project Director: Angela Uriyo

Release Consent: I hereby grant the Project Director permission to use my likeness in a photograph in any and all of its publications, including website entries, without payment or any other consideration. I understand and agree that these materials will become the property of the Project Director and will not be returned. I hereby irrevocably authorize the Project Director to edit, alter, copy, exhibit, publish or distribute this photo for purposes of publication in or for any other lawful purpose. In addition, I waive the right to inspect or approve the finished product, including written or electronic copy, wherein my likeness appears. Additionally, I waive any right to royalties or other compensation arising or related to the use of the photograph.

I hereby hold harmless and release and forever discharge the Project Director from all claims, demands, and causes of action which I, my heirs, representatives, executors, administrators, or any other persons acting on my behalf or on behalf of my estate have or may have by reason of this authorization. 
I am 21 years of age and am competent to contract in my own name. I have read this release before signing below and I fully understand the contents, meaning, and impact of this release.

(Signature)

(Date)

(Printed Name)

(Date) 


\section{APPENDIX G}

\section{OBSERVATIONAL PROTOCOL}

Site Location:

Date:

Time :

\begin{tabular}{|c|c|}
\hline Area of Observation & \\
\hline Behavior (What? By & \\
whom? Where?) & \\
\hline By whom? Where?) & \\
\hline Context (What else is & \\
going on? What is the & \\
activity? Is it a special & \\
workshop? Public or & \\
private space?) & \\
\hline participant(s) & \\
\hline Trainee or leader or & \\
\hline Type of
\end{tabular}




\begin{tabular}{|c|c|}
\hline $\begin{array}{c}\text { conveyed? By } \\
\text { whom?) }\end{array}$ & \\
\hline $\begin{array}{c}\text { Other areas of } \\
\text { observation (artifacts) }\end{array}$ & \\
\hline $\begin{array}{c}\text { Reflexive comments } \\
\text { (insights, hunches, } \\
\text { themes) }\end{array}$ & \\
\hline
\end{tabular}

Adapted from: Roller, M. R., \& Lavrakas, P. J. (2015). Applied qualitative research design: A total quality framework approach. Guilford Publications. 


\section{VITA}

Angela Uriyo attended Virginia Polytechnic Institute and State University (Virginia Tech) in Blacksburg, Virginia where she earned a Bachelor of Science in Clothing and Textiles. It was during this period that she met Dr. Carolyn Moore. Dr. Moore was a transformational leader. She was close to retirement but had lost neither her passion nor enthusiasm for teaching fashion. She led by example insisting that Angela challenge the status quo by stepping out of her comfort zone, encouraged her creativity by showing her new ways to explore her ideas, and pointing her in the direction of resources that increased her intellectual stimulation. Dr. Moore became one of Angela's greatest cheerleaders. The outcome was a skill set that expanded her horizons past the glitz and glamor of fame in the fashion industry towards a pathway that led to challenging, motivating, engaging, and transforming the way her own students learn and approach the discipline of textiles and apparel. After this critical encounter, Angela chose to pursue graduate education at Virginia Tech, majoring in Apparel Product Design and Analysis. During her graduate work, Angela was given the opportunity to be a teaching assistant in several classes in her department. After graduation, Angela landed instructor positions at the Art Institute of Dallas and the Art Institute of Pittsburg/Online, teaching fashion design and fashion marketing. During this time, Angela also pursued international freelance opportunities that utilized her technical design skills. To fulfill her dream of becoming a university professor, that Dr. Moore had sparked, Angela enrolled in the doctoral program in Human Environmental Sciences majoring in Textiles and Apparel Management at the University of Missouri-Columbia. During her doctoral journey at Mizzou, Angela had the opportunity to teach and work with numerous classes, and serve 
the graduate student body both on campus and through her discipline's international research organization in the capacity of an elected official. Angela was also an adjunct instructor at Stephens College during the 2015/2016 academic year where she taught the Fashion Design Capstone course, and a Textiles course. Angela has accepted a visiting assistant professor position at West Virginia University starting in Fall 2018. 